\title{
Centrality dependence of the nuclear modification factor of charged pions, kaons, and protons in $\mathrm{Pb}-\mathrm{Pb}$ collisions at $\sqrt{s_{N N}}=2.76 \mathrm{TeV}$
}

\author{
J. Adam et al.* \\ (ALICE Collaboration) \\ (Received 7 July 2015; revised manuscript received 9 November 2015; published 25 March 2016)
}

\begin{abstract}
Transverse momentum $\left(p_{\mathrm{T}}\right)$ spectra of pions, kaons, and protons up to $p_{\mathrm{T}}=20 \mathrm{GeV} / \mathrm{c}$ have been measured in $\mathrm{Pb}-\mathrm{Pb}$ collisions at $\sqrt{s_{N N}}=2.76 \mathrm{TeV}$ using the ALICE detector for six different centrality classes covering $0 \%-80 \%$. The proton-to-pion and the kaon-to-pion ratios both show a distinct peak at $p_{\mathrm{T}} \approx 3 \mathrm{GeV} / c$ in central $\mathrm{Pb}-\mathrm{Pb}$ collisions that decreases for more peripheral collisions. For $p_{\mathrm{T}}>10 \mathrm{GeV} / c$, the nuclear modification factor is found to be the same for all three particle species in each centrality interval within systematic uncertainties of $10 \%-20 \%$. This suggests there is no direct interplay between the energy loss in the medium and the particle species composition in the hard core of the quenched jet. For $p_{\mathrm{T}}<10 \mathrm{GeV} / c$, the data provide important constraints for models aimed at describing the transition from soft to hard physics.
\end{abstract}

DOI: 10.1103/PhysRevC.93.034913

\section{INTRODUCTION}

In ultrarelativistic heavy-ion collisions, a strongly interacting deconfined medium of quarks and gluons is created. Experimental evidence for this state of matter has been found at both the Relativistic Heavy Ion Collider (RHIC) [1-4] and the CERN Large Hadron Collider (LHC) [5-9]. Transverse momentum $\left(p_{\mathrm{T}}\right)$ spectra probe many different properties of this medium. At low $p_{\mathrm{T}}(\lesssim 2 \mathrm{GeV} / c)$ the spectra provide information on bulk production, while at high $p_{\mathrm{T}}(\gtrsim 10 \mathrm{GeV} / c)$ transport properties of the medium can be studied via jet quenching [10-12]. The microscopic QCD processes are different at low and high $p_{\mathrm{T}}$, and it is an open question if additional physics processes occur in the intermediate $p_{\mathrm{T}}$ region $\left(2 \lesssim p_{\mathrm{T}} \lesssim 10 \mathrm{GeV} / c\right)$. In this paper, the centrality evolution of the transverse momentum spectra of pions, kaons, and protons as a function of $p_{\mathrm{T}}$ for $\mathrm{Pb}-\mathrm{Pb}$ collisions at $\sqrt{s_{N N}}=$ $2.76 \mathrm{TeV}$ is presented. The focus is on intermediate and high $p_{\mathrm{T}}$, where these measurements allow comparison between baryons and mesons, strange and nonstrange particles, and the search for particle mass-dependent effects.

For inclusive charged-particle $p_{\mathrm{T}}$ spectra, jet quenching leads to a suppression of high- $p_{\mathrm{T}}$ particle production at the RHIC [13-15] and over an extended $p_{\mathrm{T}}$ range, up to $100 \mathrm{GeV} / c$, at the LHC [6,16-18]. The microscopic mechanism of jet quenching is not completely understood, and one of the main goals of the experimental programs at the RHIC and the LHC is to identify additional signatures associated with the jet quenching to constrain theoretical modeling. Particle identification (PID) is of fundamental interest because, owing to the color Casimir factor, gluons interact two times stronger with the medium than quarks $[19,20]$ and it is known from $e^{+} e^{-}$studies of three-jet events that gluons are more likely to fragment to leading baryons than quarks are [21]. In addition,

${ }^{*}$ Full author list given at the end of the article.

Published by the American Physical Society under the terms of the Creative Commons Attribution 3.0 License. Further distribution of this work must maintain attribution to the author(s) and the published article's title, journal citation, and DOI. some models for jet quenching predict large particle-speciesdependent effects [22-24]. Measurements at the RHIC, in particular for baryons, have so far been inconclusive owing to the limited $p_{\mathrm{T}}$ range and the large systematic and statistical uncertainties [25-27].

In the intermediate transverse momentum regime, the baryon-to-meson ratios, e.g., the proton yield divided by the pion yield, measured by experiments at the RHIC revealed a, so far, not well understood enhancement [28-30]. This so-called "baryon anomaly" could indicate the presence of new hadronization mechanisms such as parton recombination [3133 ] that could be significantly enhanced and/or extended out to higher $p_{\mathrm{T}}$ at the LHC owing to larger minijet production [34]. In recombination models, the enhancement at intermediate $p_{\mathrm{T}}$ is an effect of the coalescence of lower $p_{\mathrm{T}}$ quarklike particles that leads to a larger production of baryons than mesons. In a model without new intermediate $p_{\mathrm{T}}$ physics, the rise of the baryon-to-meson ratio is attributable to hydrodynamics and the decrease is solely a consequence of the growing importance of fragmentation.

In a recent letter [35] ALICE reported the charged pions, kaons, and proton $p_{\mathrm{T}}$ spectra for $p p$ and the most central and most peripheral $\mathrm{Pb}-\mathrm{Pb}$ collisions. The main observation was that, within statistical and systematic uncertainties, the nuclear modification factor is the same for $p_{\mathrm{T}}>10 \mathrm{GeV} / c$ for all three particle species. This suggests that there are no significant particle-species-dependent effects related to the energy loss. In this paper, the analysis used to obtain the measurements at high $p_{\mathrm{T}}$ is presented in full detail, and the results for all centrality classes are included. Recent measurements at low and intermediate $p_{\mathrm{T}}$ of identified particle production and correlations in $p$ - $\mathrm{Pb}$ collisions have revealed phenomena typically associated with fluidlike behavior in heavy-ion collisions [36-38]. This raises questions of whether hydrodynamics and/or recombination can also be applied to describe these small systems [39-41]. The centrality evolution studies for $\mathrm{Pb}-\mathrm{Pb}$ collisions can therefore also be seen as a possible experimental interconnection between the smallest and the largest QCD bulk systems.

The outline of this paper is as follows. In Sec. II, the data analysis is described. The method using the energy loss in 
the TPC for particle identification is laid out first and then the procedure using the Cherenkov angle measured by the high-momentum particle identification detector (HMPID) is presented. In Sec. III, the final spectra are presented, and the particle ratios and nuclear modification factors are discussed and compared with theoretical calculations and results from previous experiments at lower center-of-mass energies.

\section{DATA ANALYSIS}

The results reported in this paper have been obtained with the central barrel of the ALICE detector, which has full azimuthal coverage around midrapidity, $|\eta|<0.8$ [42]. Different particle identification (PID) devices are used for the identification of $\pi^{ \pm}, K^{ \pm}$, and $p(\bar{p})$ (see Table IV for exact $p_{\mathrm{T}}$ ranges). Ordering by $p_{\mathrm{T}}$, from lowest to highest, the results are obtained using the specific energy loss, $d E / d x$, in the silicon inner tracking system (ITS), the $d E / d x$ in the time projection chamber (TPC), the time of flight measured by the time-of-flight (TOF) detector, the Cherenkov angle measured by the HMPID, and the TPC $d E / d x$ in the relativistic rise region. The general performance of these devices is reported in Ref. [43]. Detailed description of the lower $p_{\mathrm{T}}$ analyses and the resulting $\pi^{ \pm}, K^{ \pm}$, and $p(\bar{p}) p_{\mathrm{T}}$ spectra in $\mathrm{Pb}-\mathrm{Pb}$ collisions are already published [44]. In this section, the method used to extract these $p_{\mathrm{T}}$ spectra in the HMPID and the TPC $d E / d x$ relativistic rise analysis is described in detail.

Owing to the limited acceptance of the HMPID, the analysis has been performed with the larger 2011 data set where a centrality trigger was used, restricting the HMPID results to $0 \%-50 \%$ central $\mathrm{Pb}-\mathrm{Pb}$ collisions.

\section{A. TPC $d E / d x$ relativistic rise analysis}

The relativistic rise of the $d E / d x$ in the TPC, where the average energy loss increases as $\ln \beta \gamma(3 \ll \beta \gamma \ll 1000)$, allows ALICE to extend the PID of $\pi^{ \pm}, K^{ \pm}$, and $p(\bar{p})$ up to $p_{\mathrm{T}}=$ $20 \mathrm{GeV} / c$. This section focuses on details of this analysis.

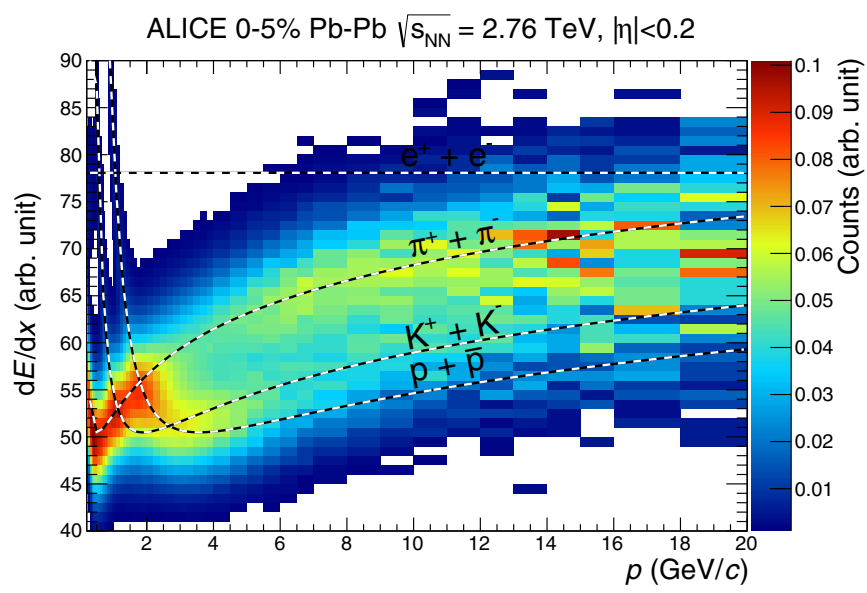

\section{Event and track selection}

The event and track selection follows closely that of the inclusive charged particle analysis [16]. The same spectrum normalization is adopted so that the systematic uncertainties related to event and track selection are common, allowing a precise comparison between the nuclear modification factors for inclusive and identified charged particles. The analysis with PID described here has additional systematic uncertainties related to the particle identification that we describe in Sec. II A 9.

A total of $11 \times 10^{6} \mathrm{~Pb}-\mathrm{Pb}$ collision events recorded in 2010 are used in this analysis. The online (offline) trigger for minimum bias interactions in $\mathrm{Pb}-\mathrm{Pb}$ collisions requires signals in two (three) of the three following detector elements: the silicon pixel detector (SPD) layers of the ITS and the two forward scintillators (V0) located on opposite sides of the interaction point. The centrality is determined from the measured amplitude in the V0 detector [45].

Primary tracks are reconstructed in the ALICE TPC [46] from clusters in up to 159 pad rows, where each cluster consists of a group of cells covering a few neighboring pads and time bins. The tracks used in the analysis are restricted to $|\eta|<0.8$ to be fully contained in the TPC active volume. Furthermore, tracks are required to have at least one hit in one of the two innermost SPD layers of the ITS, and the distance of closest approach to the primary vertex is required to be less than $2 \mathrm{~cm}$ along the beam axis and less than 7 standard deviations in the transverse plane $(\approx 350 \mu \mathrm{m}$ for tracks with $p_{\mathrm{T}}=2 \mathrm{GeV} / c$, decreasing slightly with $p_{\mathrm{T}}$ ). The resulting relative $p_{\mathrm{T}}$ resolution for these tracks is better than $5 \%$ at $p_{\mathrm{T}}=20 \mathrm{GeV} / c$ [16]. The $p_{\mathrm{T}}$ spectra have been corrected for this resolution using an unfolding procedure for $p_{\mathrm{T}}>10 \mathrm{GeV} / c[16,47]$. This correction is smaller than $2 \%$ at $p_{\mathrm{T}}=20 \mathrm{GeV} / c$.

\section{Particle identification at large transverse momentum}

Figure 1 shows the $d E / d x$ as a function of momentum $p$ in $0 \%-5 \%$ and $60 \%-80 \%$ central $\mathrm{Pb}-\mathrm{Pb}$ collisions. It is evident

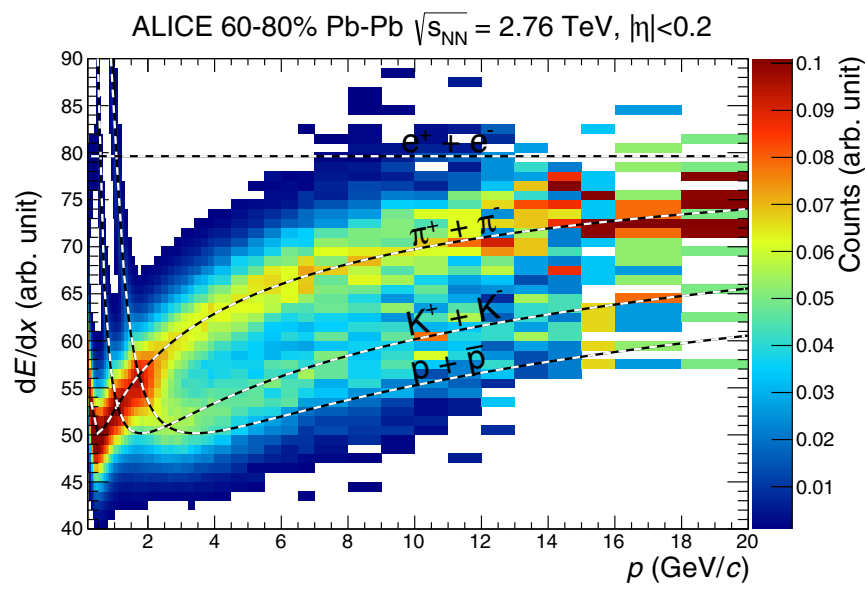

FIG. 1. The $d E / d x$ as a function of the momentum $p$ at midrapidity $|\eta|<0.2$ for $0 \%-5 \%$ (left) and $60 \%-80 \%$ (right) $\mathrm{Pb}-\mathrm{Pb}$ collisions. In each momentum bin, the $d E / d x$ spectra have been normalized to have unit integrals and only bins with more than $0.1 \%$ of the counts are shown (making electrons not visible in this plot except at very low momentum). The curves show the final $\langle d E / d x\rangle$ responses for pions, kaons, and protons. 


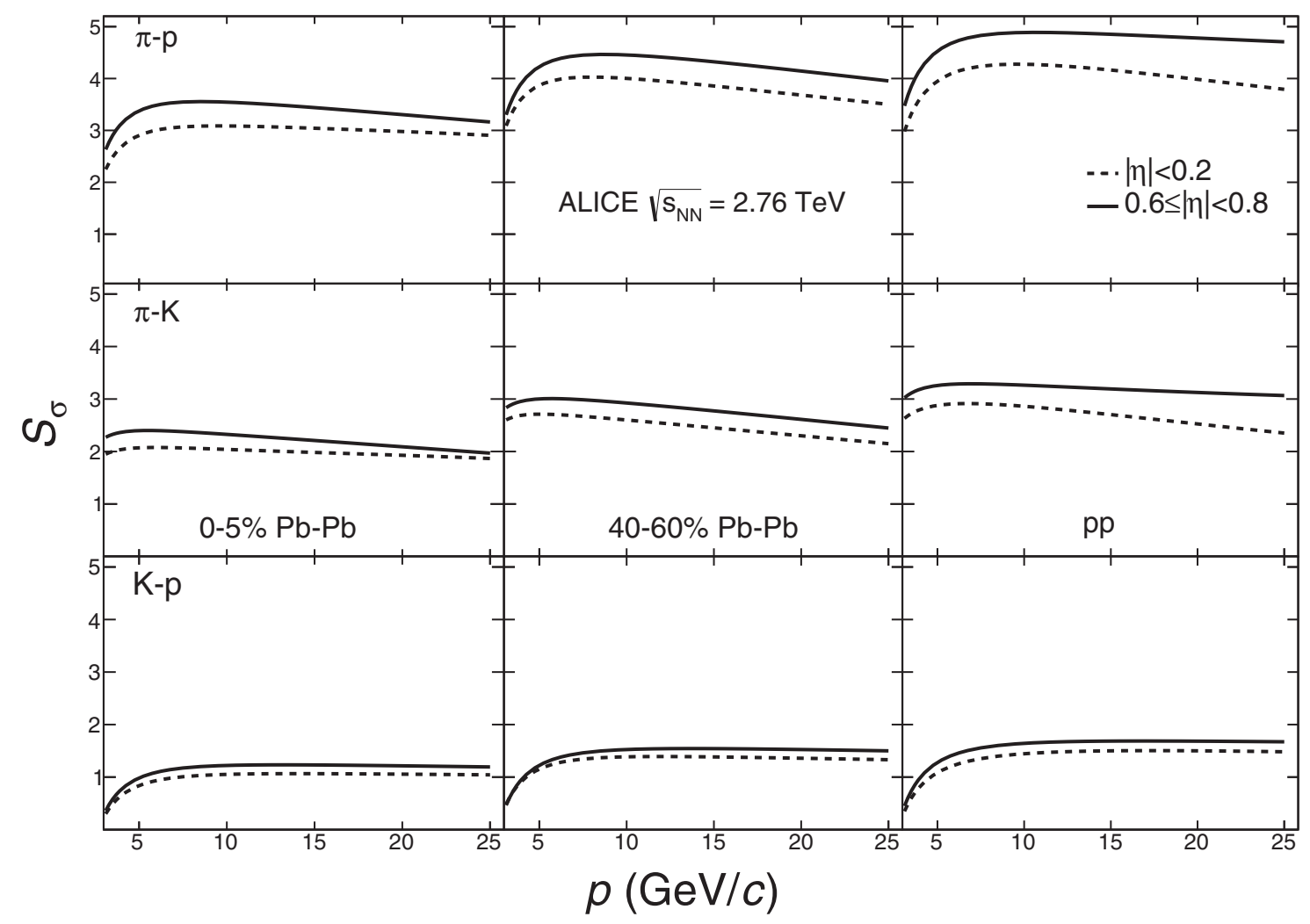

FIG. 2. Separation in number of standard deviations $\left(S_{\sigma}\right)$ as a function of momentum between pions and protons (top row), pions and kaons (middle row), and kaons and protons (bottom row). Results are shown for $0 \%-5 \%$ (left column) and 40\%-60\% (middle column) $\mathrm{Pb}-\mathrm{Pb}$ and $\mathrm{pp}$ (right column) collisions. Because the TPC response is track-length dependent, the separation is better for tracks at forward pseudorapidities (solid lines) than for those at smaller $\eta$ (dashed lines). The degradation in separation power in more central collisions is expected from occupancy effects; in the most peripheral collisions an average of 149 clusters are assigned to tracks with $p_{\mathrm{T}}>2 \mathrm{GeV} / c$, while in the most central collisions only 127 clusters are assigned.

that particle identification in the relativistic rise region requires precise knowledge of the $\langle d E / d x\rangle$ response and resolution $\sigma$. To quantify this, and to motivate the detailed studies in the following, the final response functions are used to estimate the separation power, where, for example, the charged pionto-kaon separation in number of standard deviations, $S_{\sigma}$, is

$$
S_{\sigma}=\frac{\left\langle\frac{d E}{d x}\right\rangle_{\pi^{+}+\pi^{-}}-\left\langle\frac{d E}{d x}\right\rangle_{K^{+}+K^{-}}}{0.5\left(\sigma_{\pi^{+}+\pi^{-}}+\sigma_{K^{+}+K^{-}}\right)},
$$

that is, the absolute $\langle d E / d x\rangle$ difference normalized to the arithmetic average of the resolutions. Figure 2 shows that the separation power between particle species is only a few standard deviations, making PID very challenging, requiring optimization of the $d E / d x$ signal itself and the use of external PID constraints to calibrate the response. In the following, these analysis aspects are covered in detail.

\section{The $d E / d x$ calibration}

The $d E / d x$ is obtained as a truncated mean, where the average is performed considering only the $60 \%$ lowest cluster charge values to remove the tail of the Landau-like cluster charge distribution. It is customary to use the notation $d E / d x$ and talk about the Bethe-Bloch curve, even if the $d E / d x$ used in the analysis is only the truncated mean and does not contain energy losses deposited as sub-ionization-threshold excitations or the full ionization from $\delta$ electrons, discussed in detail in Ref. [48]. While the Bethe-Bloch specific energy loss depends only on $\beta \gamma=p / m$, the one obtained from the detected truncated mean also depends on other parameters such as the actual cluster sample length, i.e., the pad length and/or track inclination over the pad. In the following, we refer to the relationship between the two types of specific energy losses as the transfer function and it is this relationship that is optimized in the $d E / d x$ calibration and used also as input for the analysis strategy discussed later.

Each of the up to 159 clusters used to reconstruct a track contains information on the ionization energy loss in the TPC. To equalize the gain, each individual readout channel has been calibrated using ionization clusters produced by the decay of radioactive krypton, ${ }_{36}^{86} \mathrm{Kr}$, released into the TPC gas [46].

In $p p$ collisions the cluster integrated charge is used for calculating the $d E / d x$. The integrated charge is corrected for the tails of the charge distribution that are below the readout threshold. Owing to the large probability for overlapping clusters in $\mathrm{Pb}-\mathrm{Pb}$ collisions, the maximum charge in the cluster is used to calculate the $d E / d x$ in this case. The maximum charge is the largest charge in a cluster cell (pad and time bin). The maximum charge has to be corrected for the drift-length-dependent reduction owing to diffusion and the 
dependence on the relative pad position of the induced signal (the measured maximum charge is largest if the cluster center is also the pad center and smallest if it is between two pads).

The performance and stability of the $d E / d x$ transfer function, with respect to gain variations, is improved in the following two ways. Reconstructed space points where the charge is deposited on a single pad, that are not used for track fitting, are included in the $d E / d x$ calculation. An attempt is made to identify clusters below the readout threshold. If a row has no cluster assigned to the track but clusters were assigned in both neighboring rows, it is assumed that the cluster charge was below the readout threshold and a virtual cluster is assigned with charge corresponding to the lowest reconstructed charge cluster on the track. This virtual cluster is then included in the calculation of the truncated mean. This is similar to the strategy adopted by ALEPH, but without changing the truncation range [49].

The $\eta$ dependence of the $d E / d x$ is sensitive to corrections for the track length and the diffusion. There is also a small correction for the direct drift-length-dependent signal attenuation, owing to absorption, of ionization electrons by oxygen [46]. At $\eta=0$ the ionization electrons drift the full $250 \mathrm{~cm}$ to the readout chambers and, as a result, the signal is spread out, owing to diffusion, making threshold effects more prominent than for tracks with $\eta=0.8$. At the same time, the sampled track length is longer for the track with $\eta=0.8$ than that with $\eta=0$. The $d E / d x$ calibration is validated using pions in the minimum ionizing particle (MIP) regime and electrons in the Fermi plateau region. A clean sample of MIP pions is selected via tracks with momenta $0.4<p<0.6 \mathrm{GeV} / c$ and energy loss $0.8<(d E / d x) /\langle d E / d x\rangle_{\text {MIP }}<1.2$. A clean electron sample is obtained in the same momentum range via centrality-dependent $d E / d x$ cuts (as $S_{\sigma}$ depends on centrality) and by rejecting kaons using time-of-flight (TOF) information: $0.9<\beta_{\mathrm{TOF}}<1.1$. For both samples it is found that the $\eta$ dependence of the $\langle d E / d x\rangle$ is negligible. We note that one expects these two classes of tracks to have different sensitivity to threshold corrections. The result of the validation test for $p p$ collisions is shown in Fig. 3, which displays the $\langle d E / d x\rangle$ response as a function of $\eta$ for electrons (top panel) and pions (middle panel).

\section{Division into homogenous samples}

From studies of the transfer function, one expects a significant track-length dependence. For the "stiff" high- $p_{\mathrm{T}}$ tracks used in this analysis, the track length in the transverse bending plane is rather similar, but there is a significant $\eta$ dependence and the effect of this on the $d E / d x$ resolution is visible in Fig. 3 for the pion MIPs. This motivates performing the analysis in $|\eta|$ intervals $-|\eta|<0.2,0.2 \leqslant|\eta|<0.4$, $0.4 \leqslant|\eta|<0.6$, and $0.6 \leqslant|\eta|<0.8-$ and then combining the results.

Furthermore, tracks close to and/or crossing the TPC sector boundaries have significantly fewer clusters assigned. Because the analyzed tracks are "stiff," those tracks close to the sector boundaries can be easily rejected using a geometric cut in the azimuthal track angle $\varphi$, which excludes approximately $10 \%$ of the tracks for $p_{\mathrm{T}}>6 \mathrm{GeV} / c$. Figure 4 shows the effect of

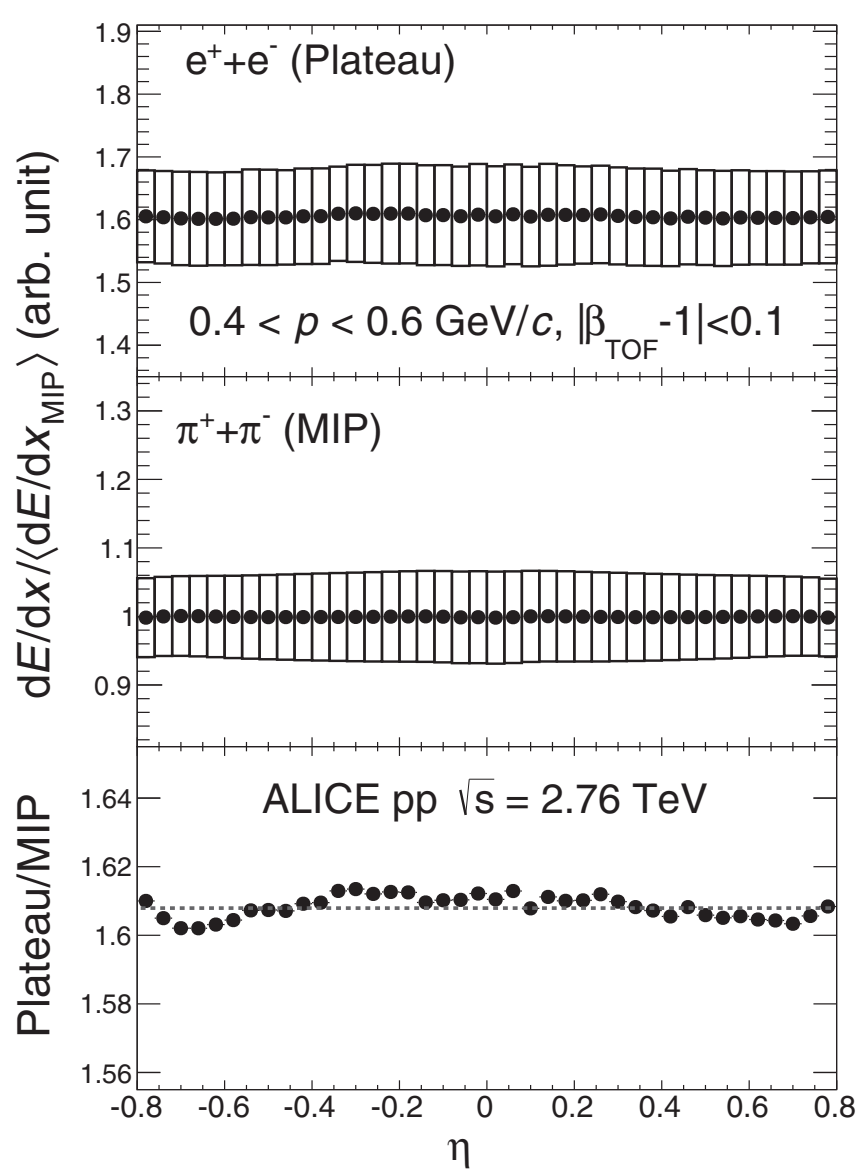

FIG. 3. The $d E / d x$ as a function of $\eta$ for electrons on the Fermi plateau (top) and MIP pions (middle); the selection criteria are described in the text. The solid round markers indicate the average, $\langle d E / d x\rangle$, and the height of the boxes is given by the standard deviation, $\sigma$. The bottom panel shows the ratio between the plateau and the MIP $\langle d E / d x\rangle$. The statistical uncertainty is smaller than the marker sizes. These results were obtained for $p p$ collisions at $\sqrt{s}=2.76 \mathrm{TeV}$.

the geometric cut on the distribution of the number of clusters per track. The cases before and after the $\varphi$ cut are shown for $p p$ (top panel) and central $\mathrm{Pb}-\mathrm{Pb}$ (bottom panel) collisions. The large difference between the distributions for $p p$ and central $\mathrm{Pb}-\mathrm{Pb}$ is an occupancy effect and essentially independent of $p_{\mathrm{T}}$. The cut significantly improves the $d E / d x$ performance by rejecting tracks with less information (fewer clusters) in regions where the calibration is more sensitive to complex edge behaviors that can have larger effects on stiff tracks. This also simplifies the analysis because in each $|\eta|$ interval, a single resolution parameter is sufficient to describe individual particles species (e.g., all pions) in a given momentum bin.

\section{Obtaining the high- $p_{\mathrm{T}}$ yields}

Because, as already mentioned, the event and track selection scheme is identical to the one used for the inclusive chargedparticle spectra [16] and each charged track has an associated TPC $d E / d x$ measurement, the charged pion, kaon, and (anti)proton yields measured in this analysis are normalized 


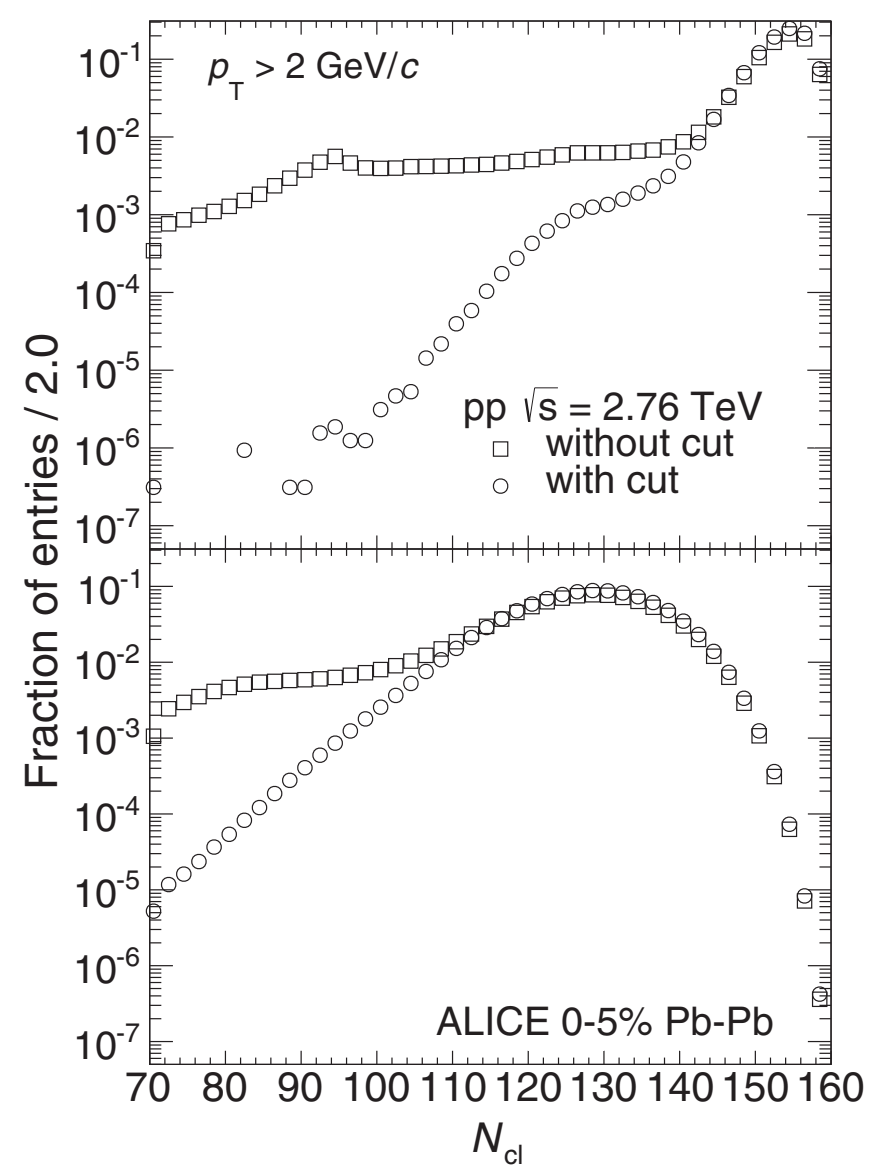

FIG. 4. Number of clusters used in the $d E / d x$ calculation for $p_{\mathrm{T}}>2 \mathrm{GeV} / c$ without (squares) and with (circles) the geometric cut. Results are presented for $p p$ (top) and central $\mathrm{Pb}-\mathrm{Pb}$ (bottom) collisions at $\sqrt{s_{N N}}=2.76 \mathrm{TeV}$. The minimum number of clusters on a track is 70 .

to the inclusive charged-particle spectra. ${ }^{1}$ This highlights the unique direct correspondence between the two analyses and guarantees that the results are fully consistent even at the level of statistical uncertainties. The analysis of the $d E / d x$ spectra is therefore aimed at extracting the relative yields of $\pi^{ \pm}, K^{ \pm}$, and $p(\bar{p})$, referred to as the particle fractions in the following.

In a narrow momentum and $|\eta|$ interval, the $d E / d x$ distribution can be described by a sum of four Gaussians $(\pi, K, p$, and $e$ ) (see, e.g., Fig. 5), and the requirements for the analysis to be able to extract the yields with high precision is that the means and widths of the Gaussians are constrained. Additional external track samples such as protons from $\Lambda$ decays are used to obtain the constraints. The method presented in the following has been benchmarked using Monte Carlo (MC) simulations and the closure tests, comparing reconstructed output with generated input, for all

\footnotetext{
${ }^{1}$ The $\varphi$ cut described in Sec. II A 4 was not applied in the inclusive charged-particle analysis, but as this cut is a geometric cut it is independent of particle species type and therefore does not affect this normalization.
}

yields show less than $2 \%$ systematic deviations. From studies comparing test beam data results with the ALICE specific MC implementation of the energy loss in the TPC, the MC is known to be precise and to take into account all important detector effects [50], with the limit that the test beam data was recorded under controlled conditions (fixed track topology and large gas gain) and that ion tail effects are not included in the MC simulations.

\section{Measurement of the TPC response: Parametrization of the Bethe-Bloch and resolution curves}

The first step of the analysis is to extract the response parametrizations used to constrain the fits. The Bethe-Bloch curve is parametrized as

$$
\left\langle\frac{d E}{d x}\right\rangle=a\left[\frac{1+(\beta \gamma)^{2}}{(\beta \gamma)^{2}}\right]^{e}+\frac{b}{c} \ln \left[\frac{(1+\beta \gamma)^{c}}{1+d^{\prime}(1+\beta \gamma)^{c}}\right],
$$

where $a, b, c, d$, and $e$ are free parameters (the variable $d^{\prime}$ is used to simplify the expression and is defined as $d^{\prime}=$ $\exp [c(a-d) / b]$, where $d$ is the $\langle d E / d x\rangle$ in the Fermi plateau regime, $\beta \gamma \gtrsim 1000$ ).

For $d^{\prime} \ll 1$, as is the case here, the parametrization has a simple behavior in different regions of $\beta \gamma$. For small $\beta \gamma, \beta \gamma \ll 3-4,\left\langle\frac{d E}{d x}\right\rangle \approx \frac{a}{(\beta \gamma)^{2 e}}$, while on the logarithmic rise, $\left\langle\frac{d E}{d x}\right\rangle \approx a+b \ln (1+\beta \gamma)$. The parametrization has been motivated by demanding this behavior in the discussed $\beta \gamma$ limits, while at the same time requiring that each parameter has a clear meaning. It uses $1+\beta \gamma$ to ensure that the logarithmic term is always positive.

The relative resolution, $\sigma /\langle d E / d x\rangle$, as a function of $\langle d E / d x\rangle$ is parametrized with a second-degree polynomial, which was found to describe the data well:

$$
\sigma /\langle d E / d x\rangle=a_{0}+a_{1}\langle d E / d x\rangle+a_{2}\langle d E / d x\rangle^{2} .
$$

The TPC response (Bethe-Bloch and resolution curves) is determined for each $\eta$ region. Owing to the deterioration of the TPC $d E / d x$ performance with increasing multiplicity, the curves differ significantly and have to be extracted separately for $p p$ and each $\mathrm{Pb}-\mathrm{Pb}$ centrality class.

The parameters $a, b, d$, and $e$ are well determined using external PID information. Secondary pion (proton) tracks identified via the reconstruction of the weak decay topology of $K_{S}^{0}(\Lambda)$ and data samples with TOF enhanced $\left(\beta_{\mathrm{TOF}}>1\right)$ primary pions are used. The $V^{0}$ selection used in this analysis is similar to the one used in the dedicated analysis [51]. To verify that the $d E / d x$ response is Gaussian, narrow invariant-mass cuts were applied to $p p$ data where the $V^{0}$ reconstruction is cleanest. Figure 6 shows single Gaussian fits to the pion and proton peaks for such data and we note that the reduced $\chi^{2}$ value is in the expected range for a valid fit model. In the following, $10 \mathrm{MeV} / c^{2}$ wide invariant-mass cuts around the peaks were used to select signal and reject background as a compromise between statistics and purity. Using this information, the Bethe-Bloch function is constrained in the $\beta \gamma$ interval of 3-60. Figure 7 shows examples of the TPC $d E / d x$ spectra for these samples in the momentum $(\eta)$ range: $5-7 \mathrm{GeV} / c(0.6 \leqslant|\eta|<0.8)$ for the most central and most peripheral $\mathrm{Pb}-\mathrm{Pb}$ collisions analyzed. In this case, the proton 

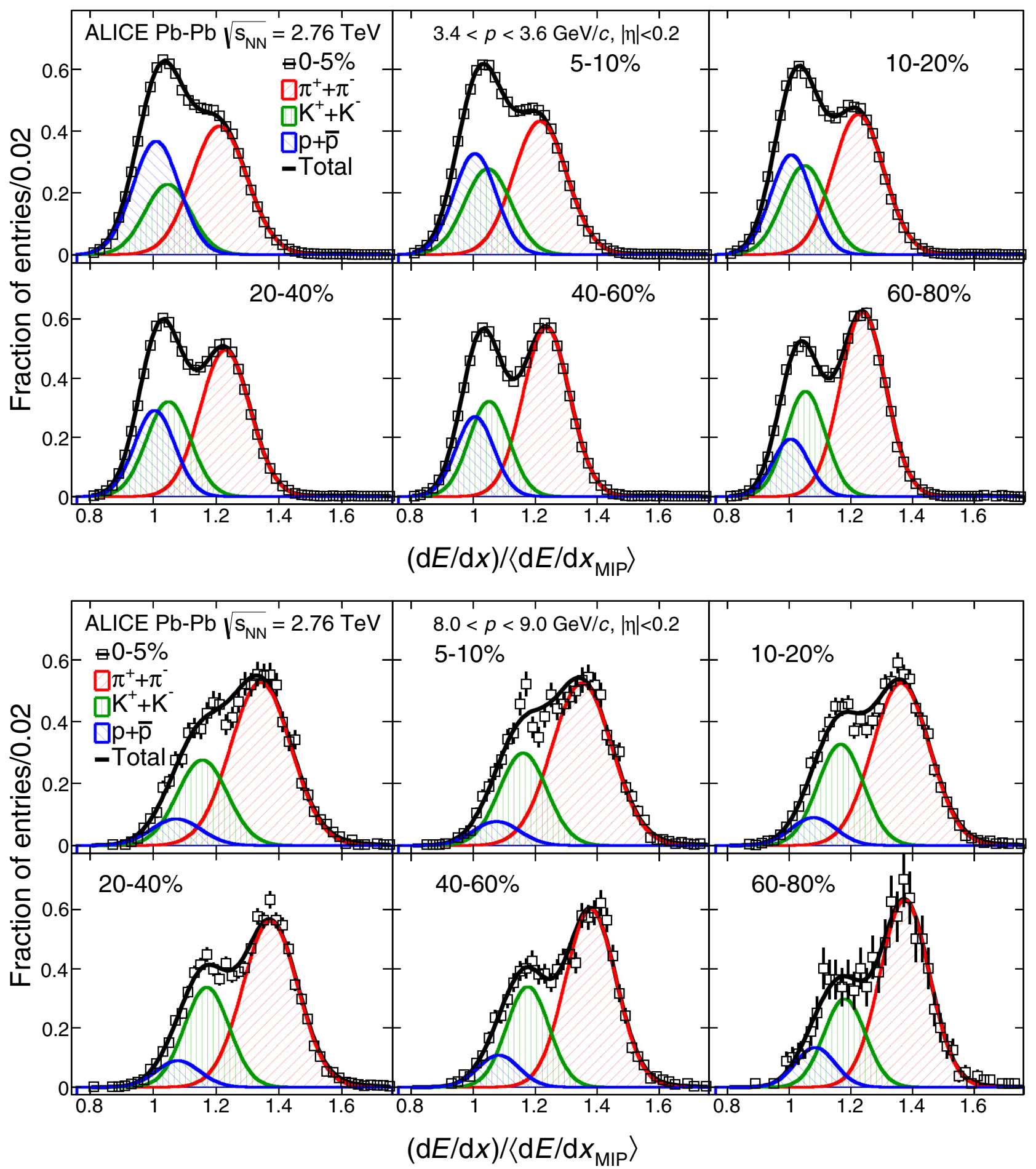

FIG. 5. Four-Gaussian fits (line) to the $d E / d x$ spectra (markers) for tracks having momentum in the range 3.4-3.6 GeV/c (top) and 8.0-9.0 GeV/c (bottom) with $|\eta|<0.2$. In each panel, the signals of pions (rightmost Gaussian), kaons, and protons (leftmost Gaussian) are shown as red, green, and blue hatched areas, respectively. The contribution of electrons is small $(<1 \%)$ and therefore not visible in the figure. Results for all six $\mathrm{Pb}-\mathrm{Pb}$ centrality classes are presented. The $d E / d x$ spectra have all been normalized to have unit integrals.

candidate samples from the $\Lambda$ decay are not pure samples and have some contamination of pions because the invariant mass peak region still contains considerable combinatorial background. This contamination is seen in the asymmetry towards the higher value of $d E / d x$ in the proton sample.
In the case of the pion samples from the $K_{S}^{0}$ decay, proton contamination creates the asymmetry towards the lower value of $d E / d x$ in the spectra. Hence, in these cases, to obtain the mean $d E / d x$ and resolution for each particle species, the asymmetric tail of the Gaussians were not considered. 


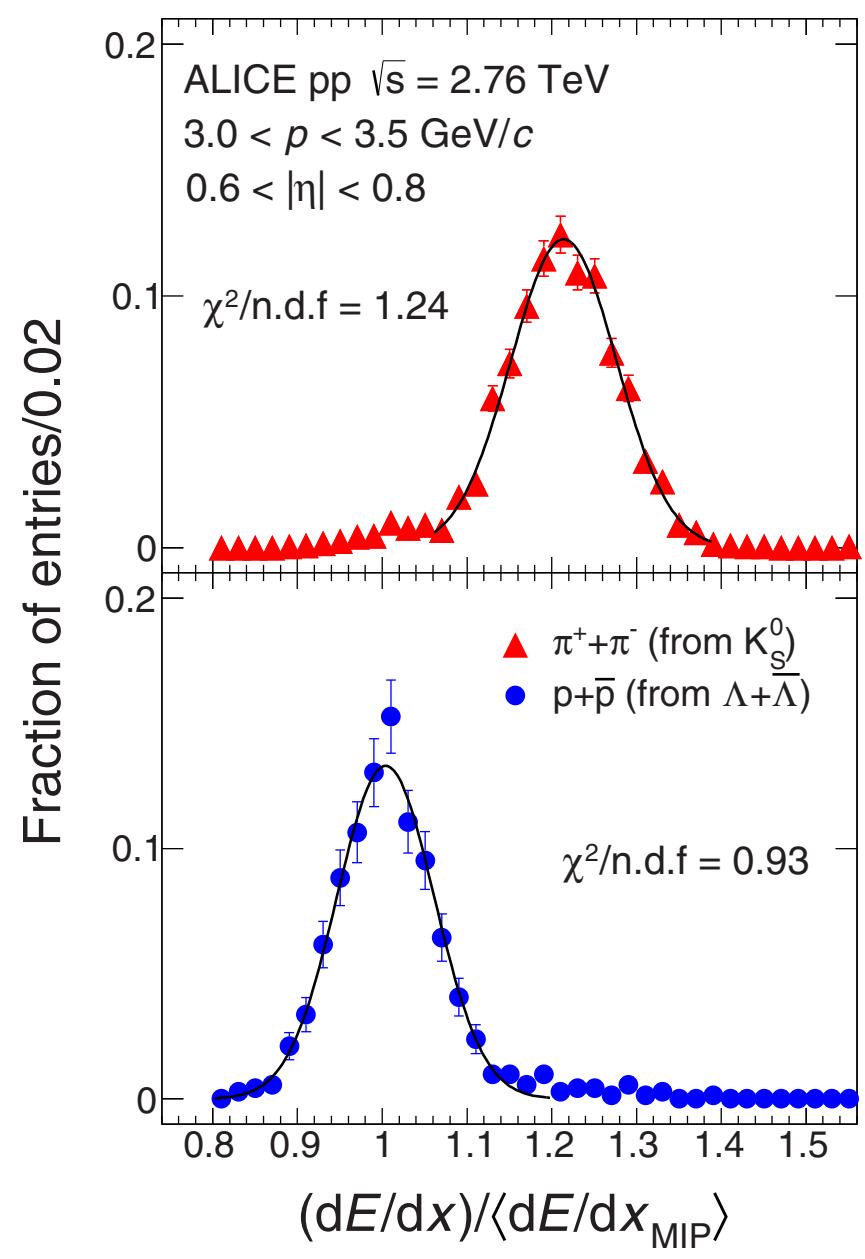

FIG. 6. $d E / d x$ spectra for secondary pions (top) and protons (bottom) identified via the reconstruction of the weak decay topology of $K_{S}^{0}$ and $\Lambda$, respectively. A narrow invariant-mass cut reducing statistics was applied to select clean samples (but the pion sample still contains a small visible proton background). The curves are single Gaussian fits to the data and the reduced $\chi^{2}$ is calculated in the range indicated by the fit curves only.

The Fermi plateau is fixed using electron-positron pairs from photon conversions (a photon conversion is reconstructed similar to a $\mathrm{V}^{0}$ decay and identified from the low invariant mass). The same information is used to measure the $d E / d x$ resolution as a function of $\langle d E / d x\rangle$. The relative resolution around the $\mathrm{MIP}^{2}$ is $\approx 5.5 \%-7.5 \%$ and improves with increasing $\langle d E / d x\rangle$ (primary ionization) in the relativistic rise region to $\approx 4.5 \%-5.5 \%$. These data samples are henceforth referred to as the external PID data.

In the relativistic rise region, the analysis is very stable because in this region $\langle d E / d x\rangle \approx a+b \ln \beta \gamma$, so the $d E / d x$ separation between particle species, e.g., protons and pions, is constant: $\langle d E / d x\rangle_{\mathrm{p}}-\langle d E / d x\rangle_{\pi} \approx a+b \ln \left(p / m_{\mathrm{p}}\right)-$ $\left[a+b \ln \left(p / m_{\pi}\right)\right] \approx b \ln \left(m_{\pi} / m_{\mathrm{p}}\right)$. So as long as all particle

\footnotetext{
${ }^{2}$ The resolution depends on centrality and track length and is worse in central events and for smaller $|\eta|$.
}

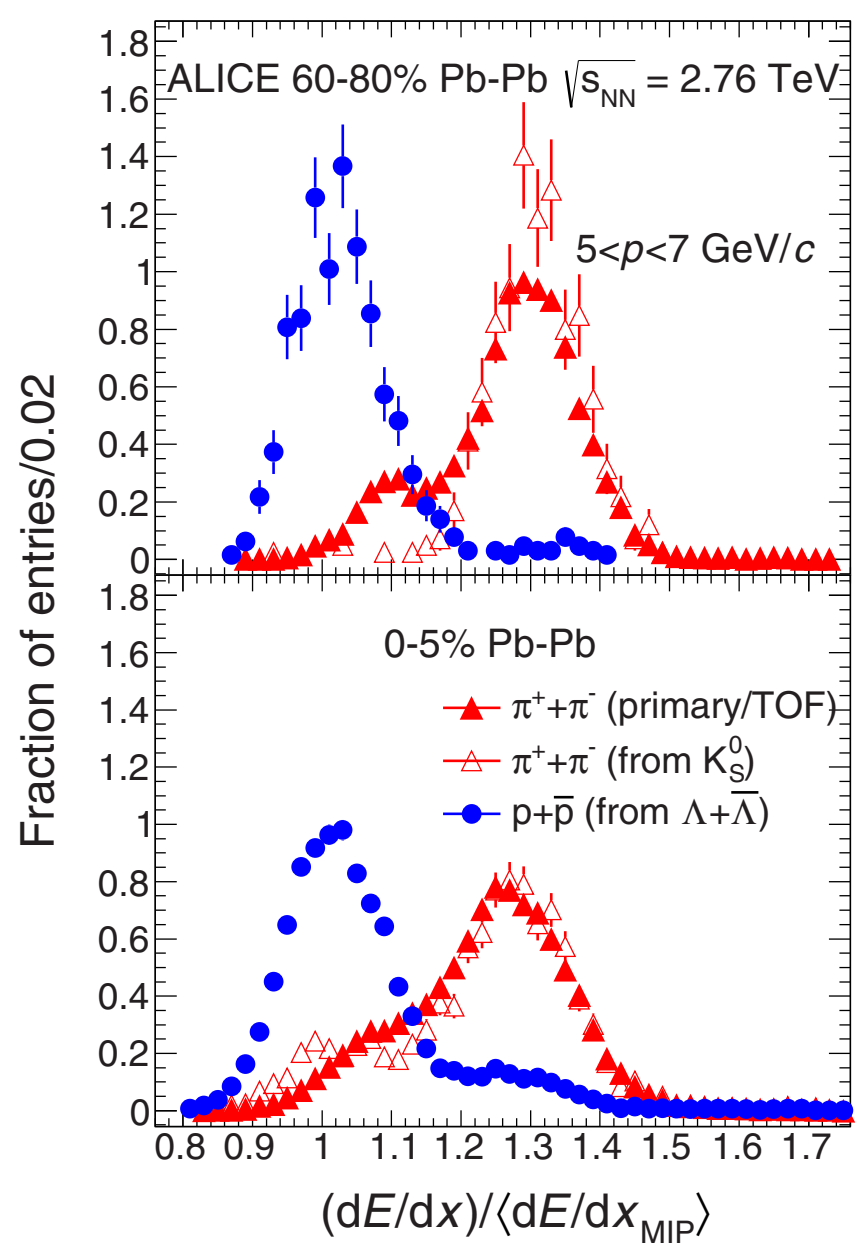

FIG. 7. $d E / d x$ spectra for secondary pions (open triangles) and protons (solid circles) identified via the reconstruction of the weak decay topology of $K_{S}^{0}$ and $\Lambda$, respectively. The spectra have been normalized to have the same integrals. The spectrum for primary pions (solid triangles) is obtained by requiring $\beta_{\mathrm{TOF}}>1$. Results for peripheral (top) and central (bottom) $\mathrm{Pb}-\mathrm{Pb}$ collisions are shown. The tracks were chosen in the momentum (pseudorapidity) interval $5<$ $p<7 \mathrm{GeV} / c(0.6 \leqslant|\eta|<0.8)$. Note that most spectra also contain a well-understood background.

species are in this $\beta \gamma$ regime a simple extrapolation can be applied. For $\beta \gamma \gtrsim 100$ the pions $(p \gtrsim 14 \mathrm{GeV} / c)$ start to approach the Fermi plateau region and the $\langle d E / d x\rangle$ dependence on $\beta \gamma$ is more complex. To address this, a two-dimensional fit to the $d E / d x$ vs $p$ distribution is performed. All the parameters of the resolution function and the parameters $a, b, d$, and $e$ of Eq. (2) are fixed. The parameter $c$ and the yields of $\pi^{+}+\pi^{-}, K^{+}+K^{-}$, and $p+\bar{p}$ in different momentum intervals are free parameters. This fit method works fine if the corrections to the logarithmic rise, owing to the transition to the plateau, are small, which restricts the current analysis to $p_{\mathrm{T}}<20 \mathrm{GeV} / c$. With higher statistics and the use of cosmic muons as additional constraints, we expect to be able to extend the method up to $50 \mathrm{GeV} / c$.

There is a final subtle point that should be mentioned here. The systematic uncertainty on the yields from the $d E / d x$ method alone is rather large for particles with ionization 

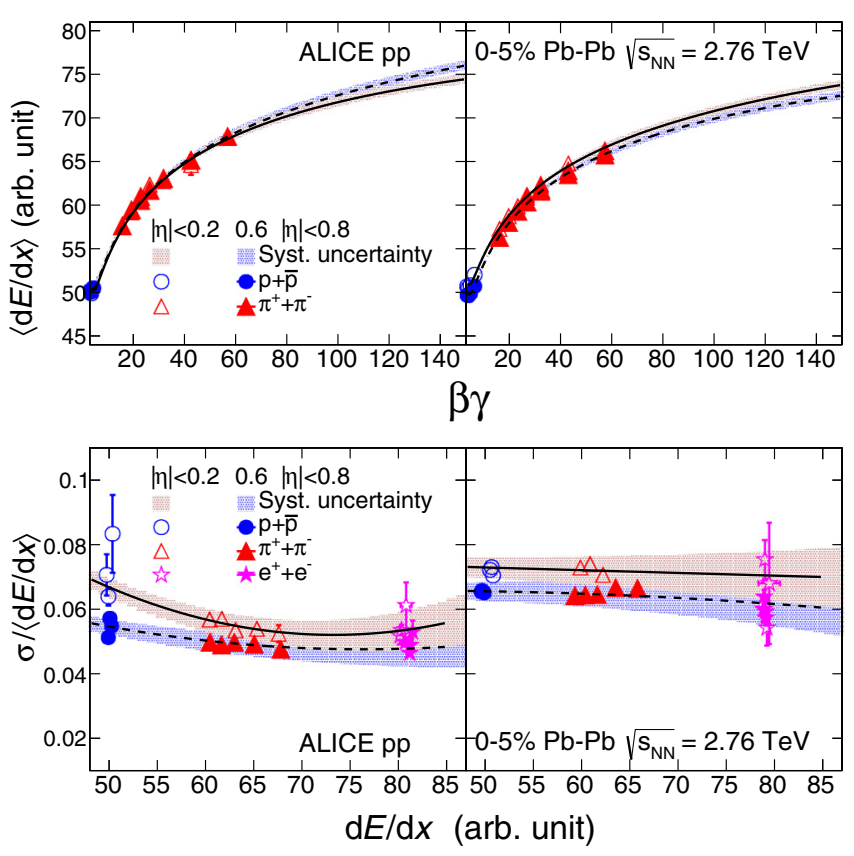

FIG. 8. Final Bethe-Bloch (top row) and resolution (bottom row) curves obtained as described in the text. Results are shown for $p p$ (left column) and $0 \%-5 \% \mathrm{~Pb}-\mathrm{Pb}$ (right column) collisions. The BetheBloch curve is shown in the region relevant for pions, kaons, and protons in this analysis. The external PID data samples of pions, protons, and electrons are used to obtain the parametrizations; these data are plotted as markers. The shaded areas represent the systematic uncertainty of the parametrizations.

energy loss close to 1 MIP, but additional information from other analyses can be used to constrain the results. One would like to avoid using the actual lower $p_{\mathrm{T}} \pi^{ \pm}, K^{ \pm}$, and $p(\bar{p})$ measurements, as this will introduce a direct bias in the final combined spectra (Sec. III). Instead, the neutral kaon yields are used to constrain the charged kaons in $\mathrm{Pb}-\mathrm{Pb}$ collisions. ${ }^{3}$ The two-dimensional fit is applied again, but the parameter $e$, which mainly affects the proton $\langle d E / d x\rangle$, is now allowed to vary while the other parameters, $a-d$, are constrained and the charged kaon yield in the fit is also restricted to be consistent with the neutral kaon yield (the pion and proton yields are free). The effect of this refit is largest in central collisions at low $p_{\mathrm{T}}$ $(<4 \mathrm{GeV} / c)$ and decreases with centrality; at $3 \mathrm{GeV} / c$ the effect on the extracted kaon yield is $10 \%(<1 \%)$ for $0 \%-5 \%$ $(60 \%-80 \%)$ collision centrality.

Figure 8 shows the final parametrizations of the BetheBloch and resolution curves for $p p$ and the most central $\mathrm{Pb}-\mathrm{Pb}$ collisions. The values obtained for the external PID data are also shown. Table I shows the values of the parameters of Eq. (2) for different centrality classes and $p p$ collisions. All parameters except $c$ are close for the four $|\eta|$ intervals and similar across systems. As previously mentioned, the parameter $c$ is related to the transition in the logarithmic

\footnotetext{
${ }^{3}$ The assumption is that the invariant $p_{\mathrm{T}}$ spectra are the same. The charged kaon fraction $\left(f_{K^{+}+K^{-}}\right)$is obtained working backwards through Eqs. (5) and (4).
}

TABLE I. Parameters obtained for the Bethe-Bloch function [Eq. (2)] for central and peripheral $\mathrm{Pb}-\mathrm{Pb}$ collisions and $p p$ collisions. Results are given as the range found for the four $|\eta|$ intervals.

\begin{tabular}{lccc}
\hline \hline Parameter & $\mathrm{Pb}-\mathrm{Pb} \mathrm{0 \% -5 \%}$ & $\mathrm{Pb}-\mathrm{Pb} \mathrm{60 \% -80 \%}$ & $p p$ \\
\hline$a$ & $33.9-35.4$ & $32.9-33.1$ & $32.5-33.3$ \\
$b$ & $7.66-7.89$ & $8.58-9.01$ & $8.52-8.77$ \\
$c$ & $2.18-7.18$ & $1.25-2.38$ & $1.65-43.0$ \\
$d$ & $78.0-78.5$ & $80.0-80.6$ & $80.6-80.7$ \\
$e$ & $1.22-1.30$ & $1.37-1.39$ & $1.43-1.55$ \\
\hline \hline
\end{tabular}

rise to the Plateau and the large difference mainly reflects that the parameter is statistically not well constrained for some of the data sets. For the $p p$ data set, where the largest variation is observed, we obtain similar results within statistical uncertainties if $c=2$ is used for all $|\eta|$ slices.

The separation power, $S_{\sigma}$, obtained with the final parametrizations for $p p, 0 \%-5 \% \mathrm{~Pb}-\mathrm{Pb}$, and $40 \%-60 \% \mathrm{~Pb}-\mathrm{Pb}$ collisions are shown in Fig. 2. As expected, the performance is the best for low-multiplicity events and decreases as the multiplicity increases and the separation is better for the longest tracks $(0.6 \leqslant|\eta|<0.8)$. For $p>6.0 \mathrm{GeV} / c$ the $S_{\sigma}$ separation is nearly constant, as expected, because of the logarithmic relativistic rise (as $\sigma \propto\langle d E / d x\rangle$ a small decrease of the separation is observed). The separation power plays an important role in the determination of the systematic uncertainties described in Sec. II A 9.

\section{Extraction of the particle fractions}

All the following results are for the sum of positive and negative pions, kaons, and protons. Positive and negative yields were found to be comparable at the 5\% level or better for all six centrality classes and $p p$ collisions.

Having determined the Bethe-Bloch and resolution curves as described in the previous section, it is now straightforward to fit the $d E / d x$ spectra using the sum of four Gaussian distributions for pions, kaons, protons, and electrons. For each momentum interval, the $\langle d E / d x\rangle$ position and width of each Gaussian are fixed. Figure 5 shows examples of these fits for the momentum intervals $3.4-3.6 \mathrm{GeV} / c$ and $8-9 \mathrm{GeV} / c$. The electrons are hardly visible in any of the fits as the yield is below $1 \%$ of the total. For $p_{\mathrm{T}}>10 \mathrm{GeV} / c$, it is no longer possible to separate electrons from pions, and the relative fraction of electrons is assumed to remain constant above this $p_{\mathrm{T}}$. There is a small contamination of primary muons in the pions owing to the similar mass (and therefore similar $\langle d E / d x\rangle$ ). High- $p_{\mathrm{T}}$ muons are predominantly the result of semileptonic decays of hadrons containing heavy quarks and for those decays one expects muon and electron branching ratios to be similar, so the electron yield (fraction) is subtracted from the pions to correct for the muon contamination. This correction changes the pion yield by less than $1 \%$ in the full $p_{\mathrm{T}}$ range, in agreement with MC simulations based on the PYTHIA generator [52]. Because this $d E / d x$ analysis is not optimized for electrons and the contamination is extrapolated to high $p_{\mathrm{T}}$, half of the correction is assigned as a systematic uncertainty. The contamination of (anti)deuterons in the (anti)proton sample is negligible $(<1 \%)$. 

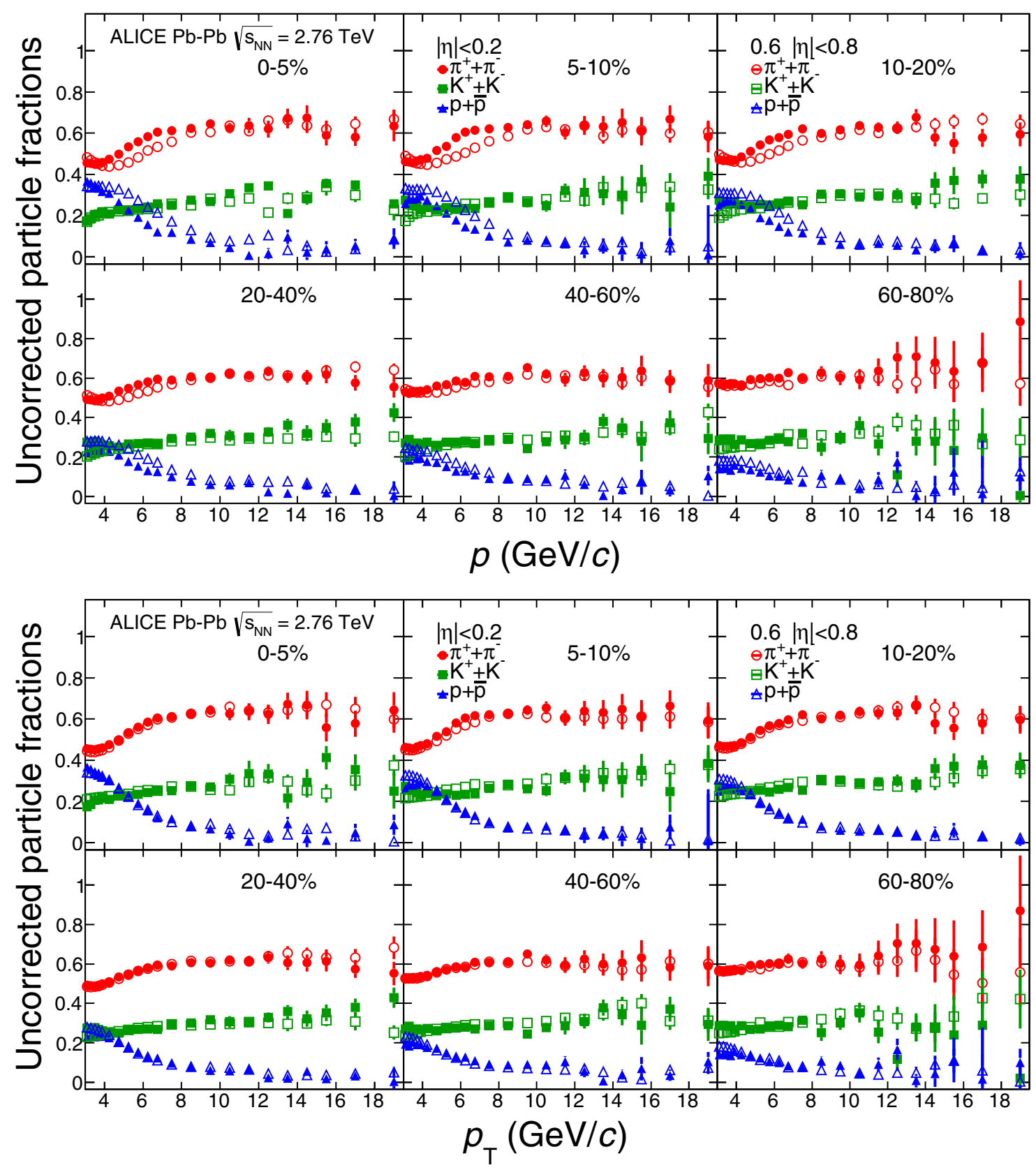

FIG. 9. Uncorrected particle fractions as a function of momentum (top) and as a function of $p_{\mathrm{T}}$ (bottom) for $|\eta|<0.2$ (solid markers) and $0.6 \leqslant|\eta|<0.8$ (open markers). Charged pions, kaons, and (anti)protons are plotted with circles, squares, and triangles, respectively. The error bars indicate the statistical uncertainty. Results for six centrality classes are presented.

The particle fractions, i.e., the contribution of charged pions $\left(f_{\pi^{+}+\pi^{-}}^{\prime}\right)$, kaons $\left(f_{K^{+}+K^{-}}^{\prime}\right)$, and (anti)protons $\left(f_{p+\bar{p}}^{\prime}\right)$ to the yield of inclusive charged particles, obtained as a function of momentum, are plotted in Fig. 9 (top) as a function of centrality for the two extreme $|\eta|$ intervals. One observes a significant $\eta$ dependence of pion and proton fractions for $p<10 \mathrm{GeV} / c$.

The extracted fractions as a function of transverse momentum are obtained bin by bin using a weighting procedure

$$
f_{\mathrm{id}}\left(\left\langle p_{\mathrm{T}}\right\rangle_{i}\right)=\sum_{j} f_{\mathrm{id}}^{\prime}\left(\langle p\rangle_{j}\right) \mathrm{R}\left(\langle p\rangle_{i},\left\langle p_{\mathrm{T}}\right\rangle_{j}\right)
$$

where $f_{\text {id }}\left(f_{\mathrm{id}}^{\prime}\right)$ is given in bins of $p_{\mathrm{T}}(p)$ and $\mathrm{R}$ is a response matrix reflecting the relation between $p$ and $p_{\mathrm{T}}$ bins. This averaging introduces some smoothing of the fractions as neighboring $p_{\mathrm{T}}$ fractions have contributions from the same $p$ fractions, but the analysis is done in narrow $|\eta|$ intervals so only a few momentum bins contribute and the fractions depend only weakly on $p$; therefore, we consider the systematic effect of this procedure negligible. The fractions $f_{\text {id }}$ are shown in the bottom panel of Fig. 9. The transformation has little effect for $|\eta|<0.2$, as expected, but we now observe that for $0.6 \leqslant|\eta|<0.8$ the results are consistent 
TABLE II. Summary of the systematic uncertainties for the charged pion, kaon, and (anti)proton spectra and for the particle ratios. The different contributions are (a) event and track selection, (b) feed-down correction, (c) correction for muons, (d) parametrization of Bethe-Bloch and resolution curves, and (e) efficiency correction (same for all systems). Note that $K / \pi=\left(K^{+}+K^{-}\right) /\left(\pi^{+}+\pi^{-}\right)$and $p / \pi=(p+\bar{p}) /\left(\pi^{+}+\pi^{-}\right)$. All values in percents.

\begin{tabular}{|c|c|c|c|c|c|c|c|c|c|c|}
\hline \multirow[b]{2}{*}{$p_{\mathrm{T}}(\mathrm{GeV} / c)$} & \multicolumn{2}{|c|}{$\pi^{+}+\pi^{-}$} & \multicolumn{2}{|c|}{$K^{+}+K^{-}$} & \multicolumn{2}{|c|}{$p+\bar{p}$} & \multicolumn{2}{|c|}{$K / \pi$} & \multicolumn{2}{|c|}{$p / \pi$} \\
\hline & 2.0 & 10 & 3.0 & 10 & 3.0 & 10 & 3.0 & 10 & 3.0 & 10 \\
\hline (a) & 8.4 & 8.1 & 8.2 & 8.1 & 8.2 & 8.1 & & & & \\
\hline (b) & \multicolumn{2}{|c|}{$<0.1$} & \multicolumn{2}{|c|}{-} & 2.1 & 1.5 & \multicolumn{2}{|c|}{$<0.1$} & 2.1 & 1.5 \\
\hline (c) & 0.1 & 1.7 & & & & & 0.6 & 1.7 & 0.6 & 1.7 \\
\hline
\end{tabular}

Pb-Pb collisions (5\%-10\%)

\begin{tabular}{|c|c|c|c|c|c|c|c|c|c|c|}
\hline (a) & 8.4 & 8.2 & 8.2 & 8.2 & 8.2 & 8.2 & \multicolumn{4}{|c|}{-} \\
\hline (d) & 1.4 & 2.2 & 16 & 8.0 & 9.5 & 16 & 18 & 10 & 9.8 & 15 \\
\hline
\end{tabular}

\begin{tabular}{|c|c|c|c|c|c|c|c|c|c|c|}
\hline (a) & 8.3 & 8.1 & 8.2 & 8.1 & 8.2 & 8.1 & \multicolumn{4}{|c|}{-} \\
\hline (d) & 1.5 & 2.3 & 16 & 8.9 & 10 & 20 & 16 & 11 & 9.2 & 18 \\
\hline
\end{tabular}

\begin{tabular}{|c|c|c|c|c|c|c|c|c|c|c|}
\hline (a) & 8.4 & 8.2 & 8.2 & 8.2 & 8.2 & 8.2 & \multicolumn{4}{|c|}{-} \\
\hline (d) & 1.5 & 2.2 & 15 & 8.4 & 10 & 17 & 16 & 11 & 10 & 17 \\
\hline
\end{tabular}

\begin{tabular}{|c|c|c|c|c|c|c|c|c|c|c|}
\hline (a) & 8.7 & 8.5 & 8.6 & 8.5 & 8.6 & 8.5 & \multicolumn{4}{|c|}{-} \\
\hline (b) & \multicolumn{2}{|c|}{$<0.1$} & & & 1.9 & 1.6 & & & 1.9 & 1.6 \\
\hline (d) & 1.4 & 2.1 & 14 & 8.0 & 11 & 17 & 15 & 10 & 11 & 17 \\
\hline
\end{tabular}

\begin{tabular}{|c|c|c|c|c|c|c|c|c|c|c|}
\hline (a) & 10 & 9.7 & 9.8 & 9.7 & 9.8 & 9.7 & \multicolumn{4}{|c|}{ - } \\
\hline (b) & \multicolumn{2}{|c|}{$\leqslant 0.1$} & & & 2.0 & 1.8 & & & 2.0 & 1.8 \\
\hline (d) & 1.4 & 2.4 & 16 & 7.1 & 20 & 29 & 16 & 8.9 & 18 & 22 \\
\hline
\end{tabular}

\begin{tabular}{|c|c|c|c|c|c|c|c|c|c|c|}
\hline (a) & 7.4 & 7.6 & 7.4 & 7.6 & 7.4 & 7.6 & \multicolumn{4}{|c|}{-} \\
\hline (b) & \multicolumn{2}{|c|}{$\leqslant 0.1$} & & & 2.0 & 1.8 & \multicolumn{2}{|c|}{$\leqslant 0.1$} & 2.0 & 1.8 \\
\hline (d) & 1.1 & 1.7 & 16 & 5.7 & 24 & 17 & 16 & 6.8 & 25 & 13 \\
\hline
\end{tabular}

with particle ratios being constant at midrapidity. We find that all four pseudorapidity intervals are consistent and the final fractions used to obtain the spectra in the next section are computed as the weighted average of the four pseudorapidity intervals.

\section{Spectra}

The invariant yields are obtained from the particle fractions using the relation

$$
\frac{d^{2} N_{\mathrm{id}}}{d p_{\mathrm{T}} d y}=J_{\mathrm{id}} \frac{\varepsilon_{\mathrm{ch}}}{\varepsilon_{\mathrm{id}}} f_{\mathrm{id}} \times \frac{d^{2} N_{\mathrm{ch}}}{d p_{\mathrm{T}} d \eta} .
$$



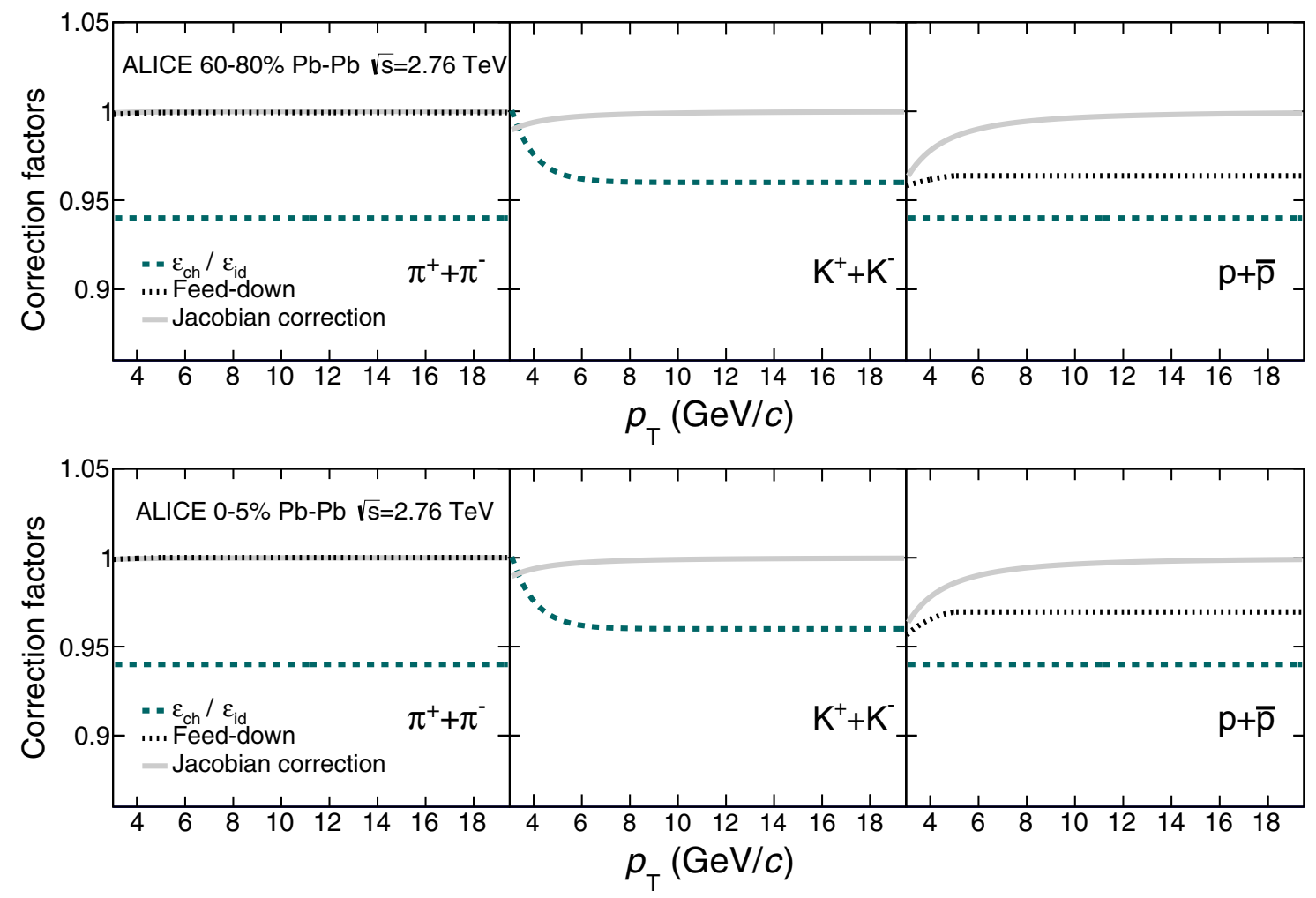

FIG. 10. Correction factors as a function of $p_{\mathrm{T}}$. These are applied to the fractions of pions (left column), kaons (middle column), and protons (right column). Results are presented for peripheral (top row) and central (bottom row) $\mathrm{Pb}-\mathrm{Pb}$ collisions. The correction to the pion fraction owing to the muon contamination is not drawn, but is $\leqslant 1 \%$. Only pions and protons are corrected for feed-down.

The first expression on the right-hand side is the input from the PID analysis, where $\left(\varepsilon_{\mathrm{ch}}\right) \varepsilon_{\text {id }}$ is the efficiency for (inclusive) identified charged particles and $J_{\text {id }}$ is the Jacobian correction (from pseudorapidity $\eta$ to rapidity $y$ ) and $f_{\text {id }}$ is the fractional yield. The second expression is the fully corrected transverse momentum spectrum of inclusive charged particles that has already been published by ALICE [16].

The relative efficiency correction, $\varepsilon_{\mathrm{ch}} / \varepsilon_{\mathrm{id}}$, was found to be consistent within $\pm 3 \%$ for all centrality classes and $p p$ collisions, and for event generators: PYTHIA [52], PHOJET [53], and HIJING [54]. Thus, an average correction was used and a systematic uncertainty of $3 \%$ was assigned. At high $p_{\mathrm{T}}$ the correction is nearly constant and on the order of 0.95 . It is below 1 because the inclusive charged-particle spectra contain weakly decaying baryons such as $\Sigma^{+}$that are not reconstructed with the charged-particle selection for primary particles. The proton and pion spectra have been corrected for feed-down from weak decays using MC simulations for the relative fraction of secondaries scaled to those extracted from distance-of-closest-approach MC template fits to data [44]. For $p_{\mathrm{T}} \approx 2(3) \mathrm{GeV} / c$, the correction is approximately $0.3 \%$ (4\%) for the pion (proton) yield and decreasing with increasing $p_{\mathrm{T}}$. Scaling between data and MC has a limited precision and could be different at higher $p_{\mathrm{T}}$. To be conservative, half of the correction is therefore assigned as a systematic uncertainty. This contribution to the systematic uncertainty is still small, as shown in Table II.
The efficiency and feed-down corrections are plotted in Fig. 10 as a function of $p_{\mathrm{T}}$ for central and peripheral $\mathrm{Pb}-\mathrm{Pb}$ collisions. The Jacobian correction from $\eta$ to $y$, which has to be included for the lower $p_{\mathrm{T}}$ bins, is also shown and the largest effect is observed for protons, as expected. At $p_{\mathrm{T}} \approx 3 \mathrm{GeV} / c$, the correction is $\approx 5 \%, \approx 1 \%$, and $\ll 1 \%$ for protons, kaons, and pions, respectively.

\section{Systematic uncertainties}

The systematic uncertainty on the invariant yields has three main components: event and track selection, efficiency correction of the fractions, and the fraction extraction. Contributions from the event and track selection are taken directly from the inclusive charged particle result [16]. The systematic uncertainties for the corrections have been covered in the previous sections and are summarized in Table II.

The systematic uncertainty on the fractions is mainly attributable to the uncertainties in the parametrization of the Bethe-Bloch and resolution curves used to constrain the fits. This systematic uncertainty can be attributable to calibration effects such that, for example, the $\langle d E / d x\rangle$ does not depend on $\beta \gamma$ alone; it can be related to the parametrizations not being able to describe the data properly, or it can be attributable to the statistical precision of the external PID data sets. To evaluate the uncertainty owing to these effects, the deviation of the fitted curves from the actual measured means and widths of 

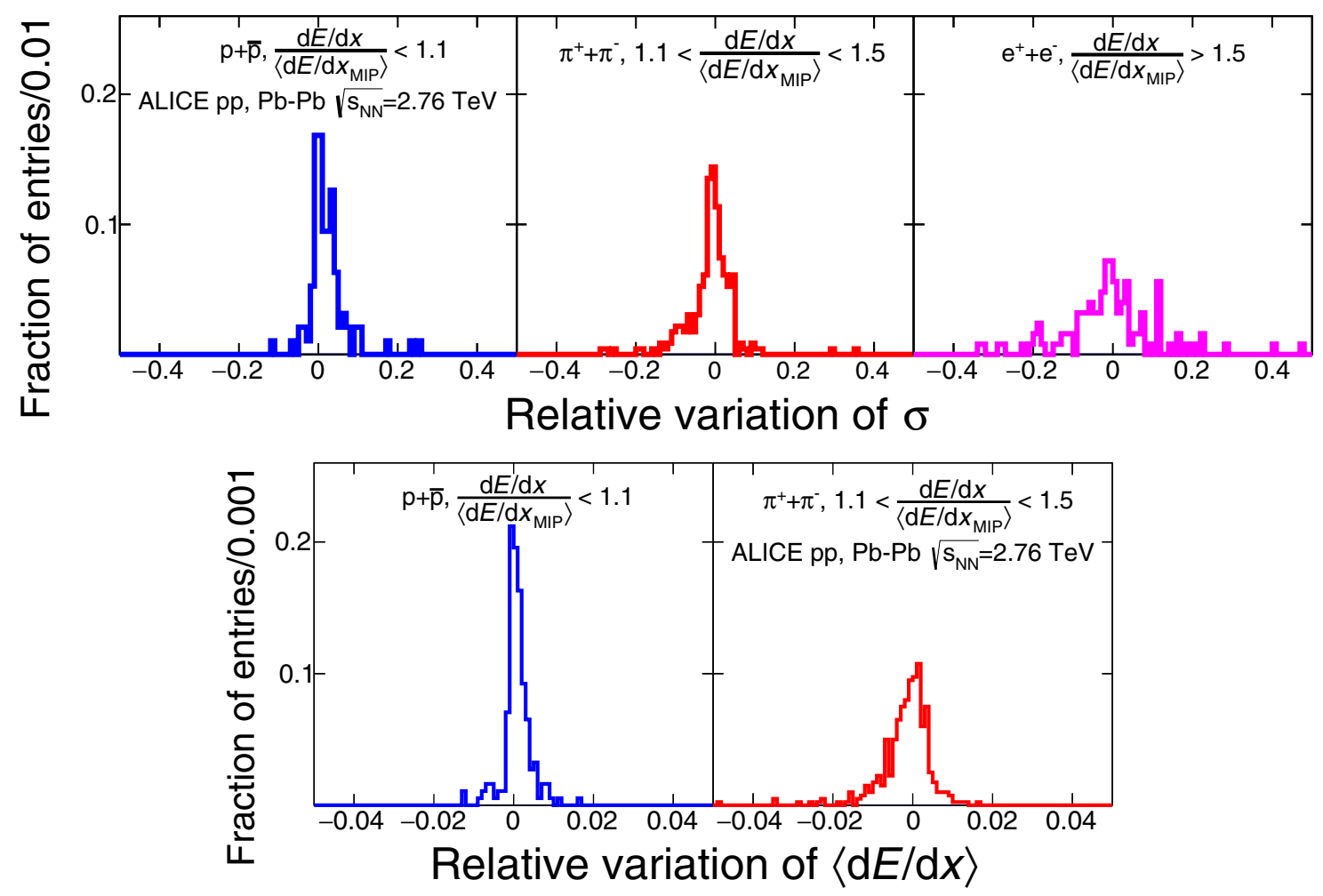

FIG. 11. (Top) Relative variation of the width parametrizations with respect to the measured values in different $d E / d x /\left\langle d E / d x_{\mathrm{MIP}}\right\rangle$ intervals. (Bottom) Relative variation of the Bethe-Bloch $\langle d E / d x\rangle$ parametrization with respect to the measured values in different $d E / d x /\left\langle d E / d x_{\mathrm{MIP}}\right\rangle$ intervals. The distributions were constructed using all the available data, six centrality classes, and $p p$ collisions with four subsamples ( $|\eta|$ intervals) each.

the $d E / d x$ spectra obtained from the analysis of the external pion, proton, and electron samples are used. Figure 11 shows the relative variations; all the available data were used for constructing the distributions; i.e., each of the six centrality classes and $p p$ collisions have four subsamples of tracks at different $|\eta|$. It was found that the precision of all these data sets is similar, so the final variation in systematic uncertainties for the same observable for different centrality classes and $p p$ collisions is caused by the different separation power shown in Fig. 2. The results for the width (Fig. 11, top) are shown for $p+\bar{p}, \pi^{+}+\pi^{-}$, and $e^{+}+e^{-}$, corresponding to the different samples and covering different $\langle d E / d x\rangle /\left\langle d E / d x_{\mathrm{MIP}}\right\rangle$ ranges. In a given $\langle d E / d x\rangle /\left\langle d E / d x_{\mathrm{MIP}}\right\rangle$ interval, the standard deviation of the distribution was taken as the systematic uncertainty associated with the extraction of the widths. An analogous analysis was done for the Bethe-Bloch curve, an example of which is shown in the bottom panel of Fig. 11.

In peripheral collisions, an additional contribution originating from the statistical uncertainty in the fits to the external PID data has to be taken into account for the Bethe-Bloch curve. The total systematic uncertainty is assigned as the quadratic sum of both contributions and is the band shown around the parametrizations in Fig. 8.

The propagation of the uncertainties to the particle fractions is done by refitting the $d E / d x$ spectra, while randomly varying the constrained parameters, $\langle d E / d x\rangle$ and $\sigma$, within the uncertainty for the parametrizations assuming a Gaussian variation centered at the nominal value. For each $p_{\mathrm{T}}$ bin, all the $\langle d E / d x\rangle$ and $\sigma$ values are randomly varied and refitted 1000 times, resulting in fraction distributions like those shown in Fig. 12. The systematic uncertainties assigned to the particle fractions are the standard deviation of the associated distributions. By using the same method for the particle ratios (Fig. 12, bottom), the correlation in the fit between the extracted yields for the two different particle species are directly taken into account. At high $p_{\mathrm{T}}$, the variation becomes dominated by statistical fluctuations owing to the limited amount of data. However, as the fractions are nearly constant there (see Fig. 9) and the separation is also nearly constant (see Fig. 2), a constant absolute systematic uncertainty is assigned for $p_{\mathrm{T}}>8 \mathrm{GeV} / c$.

A summary of the different contributions to the systematic uncertainty is shown in Table II for all centrality classes and for two representative $p_{\mathrm{T}}$ regions. For pions, the dominant contribution comes from the event and track selection, which amounts to $7 \%-8 \%$ over the whole $p_{\mathrm{T}}$ range, while the PID systematic uncertainty stays between $1 \%-2 \%$. For kaons and protons, the PID systematic uncertainty is the largest. The systematic uncertainty decreases with increasing separation and is smaller where the fractions are larger; see Fig. 9. For protons at $p_{\mathrm{T}}=3 \mathrm{GeV} / c$, the two effects largely compensate (the fractional yields increase for more central collisions) to keep the systematic uncertainty nearly constant. For kaons, 

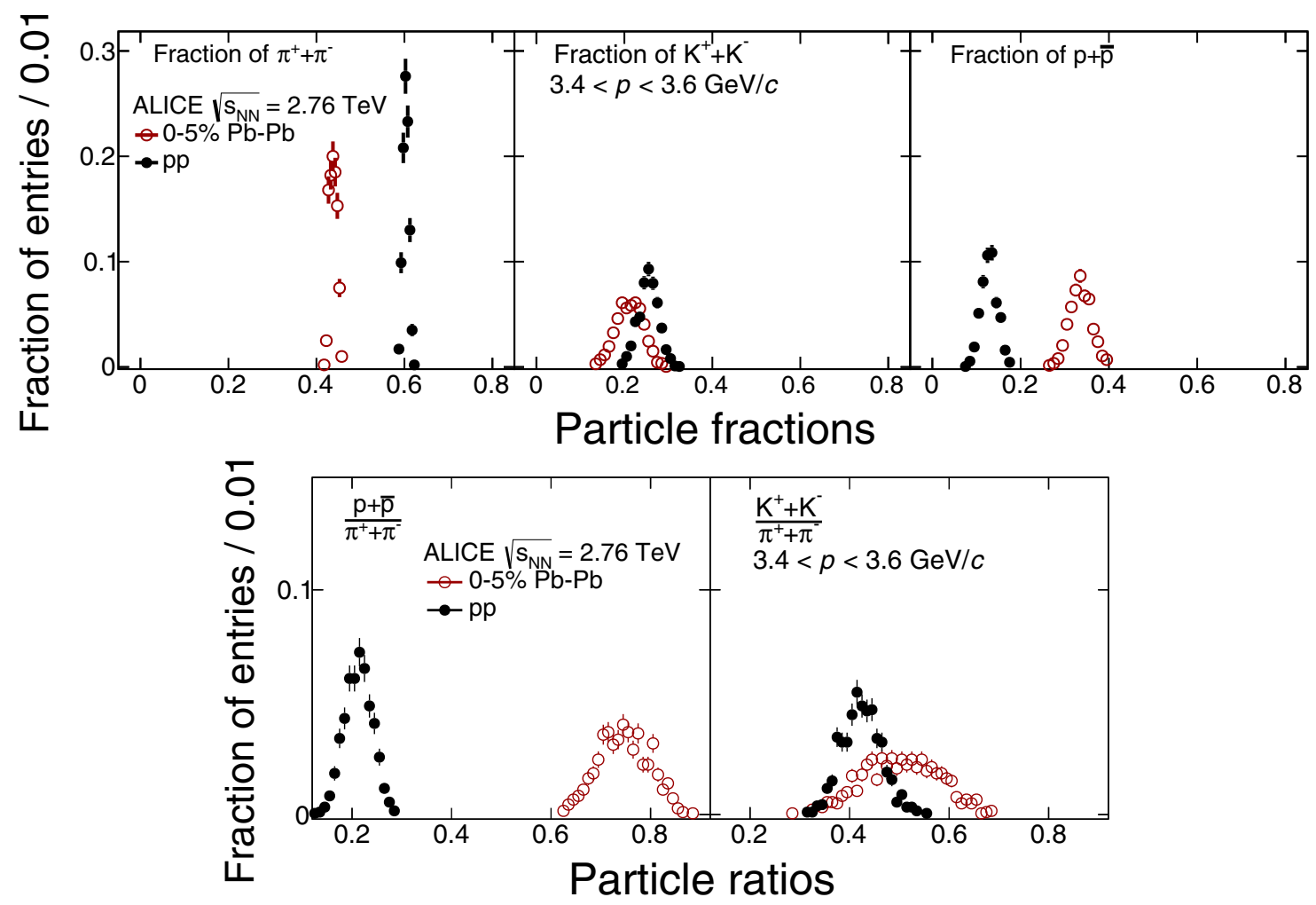

FIG. 12. An example of the systematic uncertainty estimation in $0 \%-5 \% \mathrm{~Pb}-\mathrm{Pb}$ and $p p$ collisions for $3.4<p \leqslant 3.6 \mathrm{GeV} / c$. (Top) The variation of extracted fractional yields for pions (left), kaons (middle), and protons (right) when the fixed values for the $\langle d E / d x\rangle$ and the resolution are randomly varied. (Bottom) The corresponding variation of the particle ratios.

at the same $p_{\mathrm{T}}$, there is a strong centrality dependence because the fractional yields also are lower for more central collisions. For the lower multiplicity intervals ( $p p$ and $60 \%-$ $80 \%$ centrality) this trend is broken because of the significant statistical uncertainty in the parametrized curves.

At high $p_{\mathrm{T}}(\approx 10 \mathrm{GeV} / c)$ the PID systematic uncertainty for kaons stays between $7 \%$ and $8 \%$ for $\mathrm{Pb}-\mathrm{Pb}$ collisions and is around $5 \%$ for $p p$ collisions. For protons, the contribution is $16 \%-20 \%$ (except for $60 \%-80 \% \mathrm{~Pb}-\mathrm{Pb}$ collisions, where it is $29 \%$ owing to a much larger statistical uncertainty in the fits to the external PID data).

\section{B. HMPID analysis of $\mathrm{Pb}-\mathrm{Pb}$ data}

The HMPID is used to constrain the uncertainty of the charged pion, kaon, and (anti)proton measurements in the transition region between the TOF and TPC relativistic rise methods (in the region around $p_{\mathrm{T}}=3 \mathrm{GeV} / c$ ). Thus, it both improves the precision of the measurement and validates the other methods in the region where they have the worst PID separation.

The HMPID [55] detector consists of seven identical proximity-focusing RICH (ring-imaging Cherenkov) counters. Photon and charged-particle detection is provided by a multiwire proportional chamber (MWPC) coupled to a CsI photocathode segmented into pads of size $0.8 \times 0.84 \mathrm{~cm}^{2}$ (the probability to obtain an amplified signals for an incident photon, the quantum efficiency, is $\approx 25 \%$ for $\lambda_{\text {ph }}=175 \mathrm{~nm}$ ). The amplification gas is $\mathrm{CH}_{4}$ at atmospheric pressure with an anode-cathode gap of $2 \mathrm{~mm}$; the operational voltage is $2050 \mathrm{~V}$, corresponding to a gain of $\approx 4 \times 10^{4}$. It is located at about $5 \mathrm{~m}$ from the beam axis, covering a limited acceptance of $|\eta|<0.5$ and $1.2^{\circ}<\varphi<58.5^{\circ}$.

The HMPID analysis uses the $2011 \mathrm{~Pb}-\mathrm{Pb}$ data with around $7.8 \times 10^{6}$ central triggered events $(0 \%-10 \%$ centrality) and $5 \times 10^{6}$ semicentral triggered events $\left(10 \%-50 \%\right.$ centrality $\left.^{4}\right)$. The event and track selection is similar to the one described in Sec. II A 1, but in addition it is required that the tracks are propagated and matched to a primary ionization cluster in the MWPC gap of the HMPID detector (denoted matched cluster in the following). The matching efficiency, including spurious matches, is $\approx 95 \%$ (see $\varepsilon_{\text {match }}$ below). The matching criteria are tightened to reject the fake cluster-track matches, which account for $\approx 30 \%-40 \%$ (see $C_{\text {distance }}$ later), so that only tracks matched with their corresponding primary ionization cluster are identified. The PID in the HMPID is done by measuring the Cherenkov angle, $\theta_{\mathrm{Ch}}[55]$, given by

$$
\cos \theta_{\mathrm{Ch}}=\frac{1}{n \beta} \Rightarrow \theta_{\mathrm{Ch}}=\arccos \left(\frac{\sqrt{p^{2}+m^{2}}}{n p}\right),
$$

where $n$ is the refractive index of the radiator used (liquid $\mathrm{C}_{6} \mathrm{~F}_{14}$ with $n=1.29$ at temperature $T=20^{\circ} \mathrm{C}$ for photons

\footnotetext{
${ }^{4}$ To match centrality classes with the high- $p_{\mathrm{T}}$ analysis, only spectra for $0 \%-40 \%$ are shown in this paper. Results for $20 \%-30 \%, 30 \%-$ $40 \%$, and $40 \%-50 \%$ are available on HepData.
} 


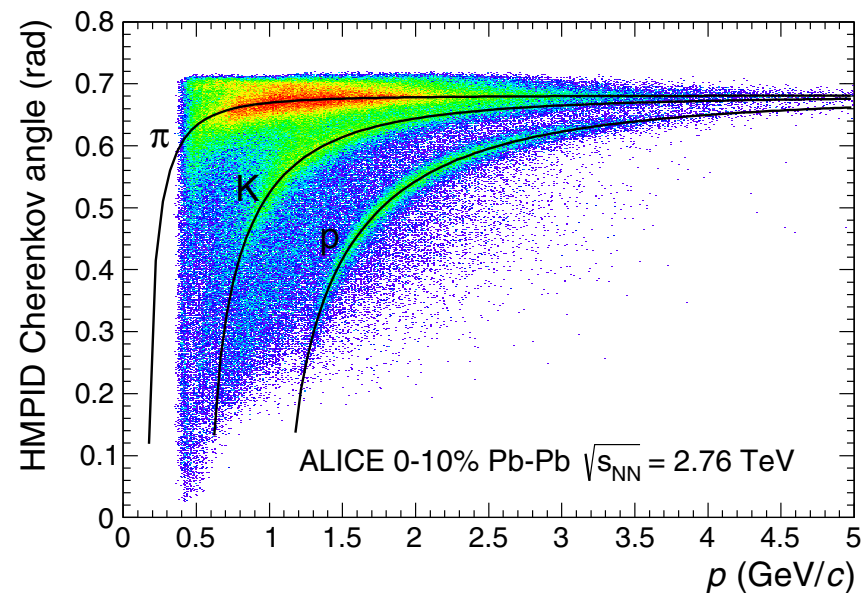

FIG. 13. Cherenkov angle measured in the HMPID as a function of the momentum $p$ in $0 \%-10 \%$ central $\mathrm{Pb}-\mathrm{Pb}$ collisions. The solid lines represent the theoretical curves for each particle species. The $z$ axis indicated by the color scale is logarithmic.

with energy $6.68 \mathrm{eV}$ ). Figure 13 shows the Cherenkov angle as a function of the momentum for central $\mathrm{Pb}-\mathrm{Pb}$ collisions.

The measurement of the single photon $\theta_{\mathrm{Ch}}$ angle in the HMPID requires knowledge of the track impact position and angle. These are estimated from the track extrapolation from the central tracking devices up to the radiator volume, where the Cherenkov photons are emitted. Only one matched cluster is associated with each extrapolated track, selected as the closest cluster to the extrapolated track point on the cathode plane, with a charge above $\approx 120 \sigma_{\text {noise }}$. The cut on the charge excludes clusters from electronic noise and photons. The matching efficiency is defined for tracks extrapolated to the HMPID acceptance as

$$
\varepsilon_{\text {match }}=\frac{N(\text { extrapolated with matched cluster })}{N(\text { extrapolated })} .
$$

This efficiency is $\approx 95 \%$ and independent of momentum, particle species, and event multiplicity.

In Fig. 14, the residuals distribution between the track extrapolation and the matched cluster position in local chamber coordinates, $X$ and $Y$, for tracks with $p_{\mathrm{T}}>1.5 \mathrm{GeV} / c$ is shown. The distributions have a resolution of $\sigma_{\text {res }} \approx 2 \mathrm{~cm}$. To reject fake cluster-match associations in the detector, mainly the situation when there is no correct signal to match, as for example the particle, was absorbed or deflected in the material between the TPC and the HMPID detector, a selection on the distance, $\sqrt{X^{2}+Y^{2}}$, computed on the cathode plane between the track extrapolation and the matched cluster is applied. This distance has to be less than $5 \mathrm{~cm}$. This represents the best compromise between the loss of statistics and the probability of an incorrect association, where the latter becomes negligible $(<0.1 \%)$ even in the most central collisions, as estimated from MC simulations. The distance cut leads to a correction

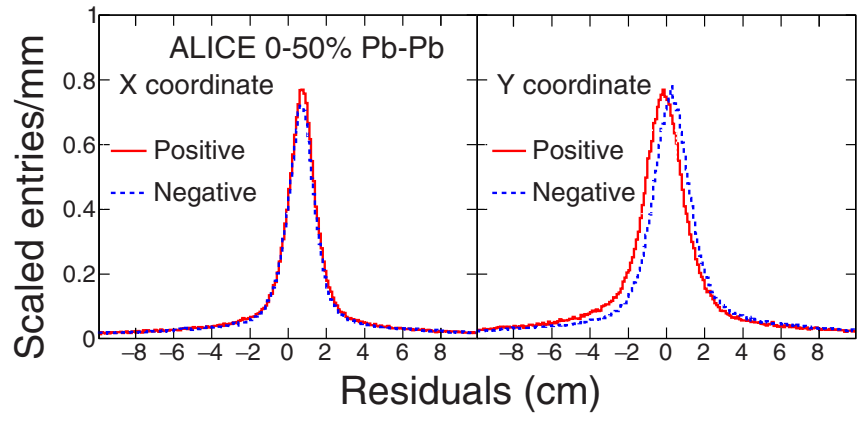

FIG. 14. Distribution of the $X$ (left) and $Y$ (right) residuals between the matched cluster position and the closest extrapolated track point at the HMPID chamber plane (HMPID module 2), for positive and negative tracks with $p_{\mathrm{T}}>1.5 \mathrm{GeV} / c$ in $\mathrm{Pb}-\mathrm{Pb}$ collisions ( $0 \%-50 \%$ centrality). The histograms have been scaled to have a similar maximum value. The small shift between positive and negative tracks in the $Y$ residuals is attributable to a radial residual misalignment and an imperfect estimate of the energy loss in the material traversed by the track and is not corrected for in the calculation of the residual distance.

factor

$$
\begin{aligned}
& C_{\text {distance }} \\
& \quad=\frac{N(\text { extrapolated with matched cluster distance }<5 \mathrm{~cm})}{N(\text { extrapolated with matched cluster })},
\end{aligned}
$$

for each momentum bin and does not depend on event multiplicity. Figure 15 shows this correction factor as a function of $p_{\mathrm{T}}$ for positive and negative tracks integrated over the centrality classes $(0 \%-50 \%)$.

Starting from the photon cluster coordinates on the photocathode, a backtracking algorithm calculates the corresponding emission angle. The Cherenkov photons are selected by the Hough transform method (HTM) [56], which for each track transforms the coordinates of photon hits into emission angles. The angle interval with the most hit candidates is selected and

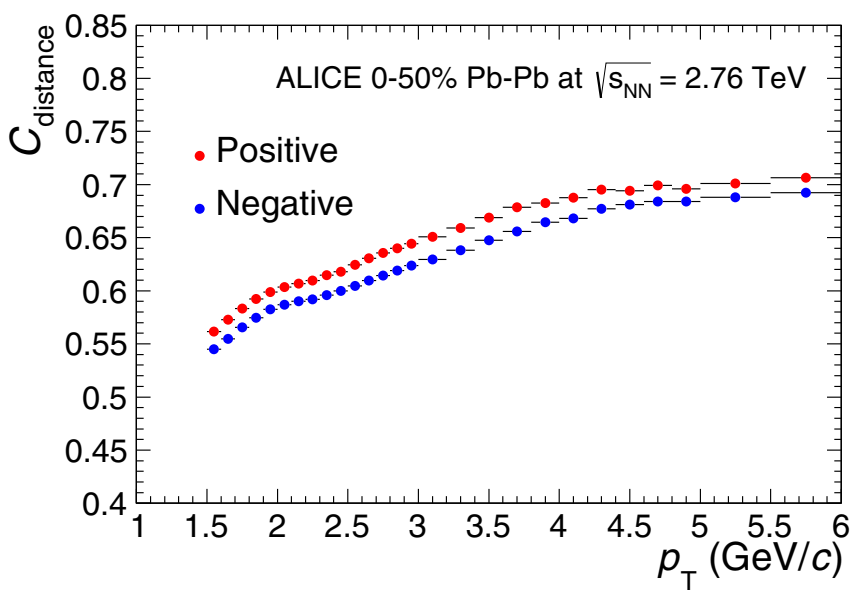

FIG. 15. The distance cut correction, $C_{\text {distance, }}$, as a function of $p_{\mathrm{T}}$ for positive (red) and negative (blue) tracks, respectively, in $\mathrm{Pb}-\mathrm{Pb}$ collisions ( $0 \%-50 \%$ centrality). 


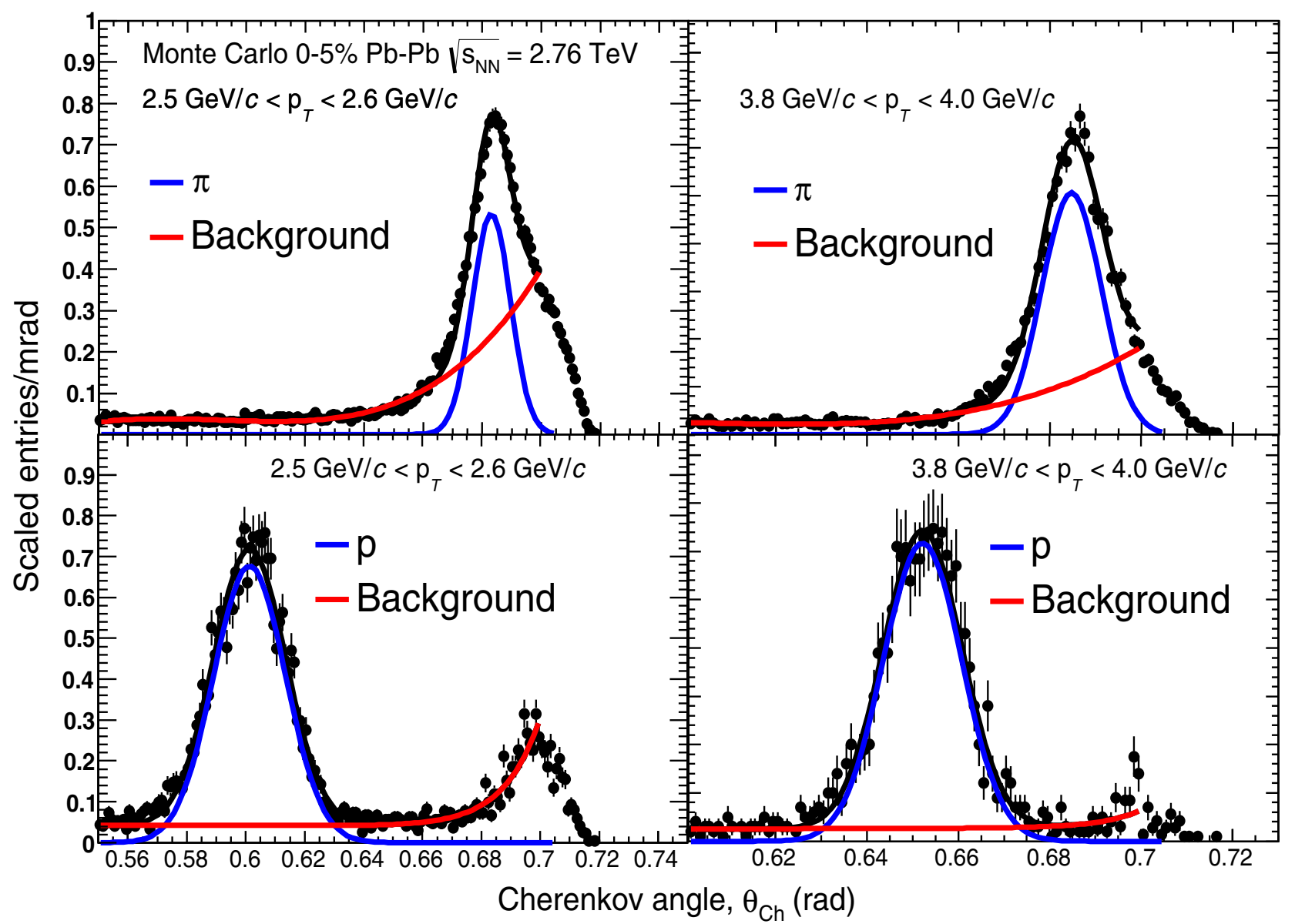

FIG. 16. Fit to the $\theta_{\mathrm{Ch}}$ distributions of pions (top) and protons (bottom) obtained in MC simulations for two different momentum bins. The histograms have been scaled to have a similar maximum value.

$\theta_{\mathrm{Ch}}$ is computed as the weighted mean of the single photon angles. In central $\mathrm{Pb}-\mathrm{Pb}$ collisions, where the total number of signals in the HMPID chambers is large, it is possible that the angle is constructed based on hits not corresponding to the Cherenkov photons associated with the track. This results in a significant reduction of the PID efficiency in the most central collisions. Figure 16 gives an example of the same effect in MC simulations. The response function consists of a Gaussian distribution for correctly assigned rings (signal) plus a distribution strongly increasing with the Cherenkov angle for incorrectly assigned rings (background). The signals from other tracks and photons in the same event are uniformly distributed on the chamber plane, and so the background rises with $\theta_{\mathrm{Ch}}$ because the probability of finding background clusters increases. The background contribution decreases with increasing track momentum because higher-momentum tracks give rise to a larger number of Cherenkov photons and have a smaller inclination angle, producing rings that are more likely to be fully contained inside the acceptance. As a result of this, the probability of incorrectly associating an angle computed from background clusters to the track decreases. The shoulder in the distribution starting at $0.7 \mathrm{rad}$ is a boundary effect owing to the finite geometrical acceptance of the chamber.
Figure 17 gives examples of the reconstructed Cherenkov angle distributions in two narrow $p_{\mathrm{T}}$ intervals for different centrality classes; the reconstructed angle distribution is fitted with a sum of three Gaussian distributions, corresponding to the signals from pions, kaons, and protons, plus a distribution associated with the misidentified tracks that is modeled with a sixth-degree polynomial function that minimizes the reduced $\chi^{2}$ of the fit.

The fitting is performed in two steps. In the first step, the initial parameters are based on the expected values. For the signal, the means $\left\langle\theta_{\mathrm{Ch}}\right\rangle_{i}$ are obtained from Eq. (6), tuning the refractive index to match the observed Cherenkov angles, and the $\sigma$ values $\sigma_{i}$ are taken from the MC distribution in the given transverse momentum bin. The initial shape of the sixth-degree polynomial background is taken from MC simulations. Furthermore, the signal parameters are constrained to the ranges $\left[\left\langle\theta_{\mathrm{Ch}}\right\rangle_{i}-\sigma_{i},\left\langle\theta_{\mathrm{Ch}}\right\rangle_{i}+\sigma_{i}\right]$ for the means and $\left[\sigma_{i}-0.1 \sigma_{i}, \sigma_{i}+0.1 \sigma_{i}\right]$ for the widths. After this first step, the $p_{\mathrm{T}}$ dependence of each parameter is fitted with a continuous function. In the second step, the fitting is repeated with only the yields as free parameters and constraining the mean and $\sigma$ values to the continuous functions. The means and widths constrained in this way are all found to be independent of centrality, as shown in Fig. 18 for $0 \%-5 \%$ and $40 \%-50 \%$ 

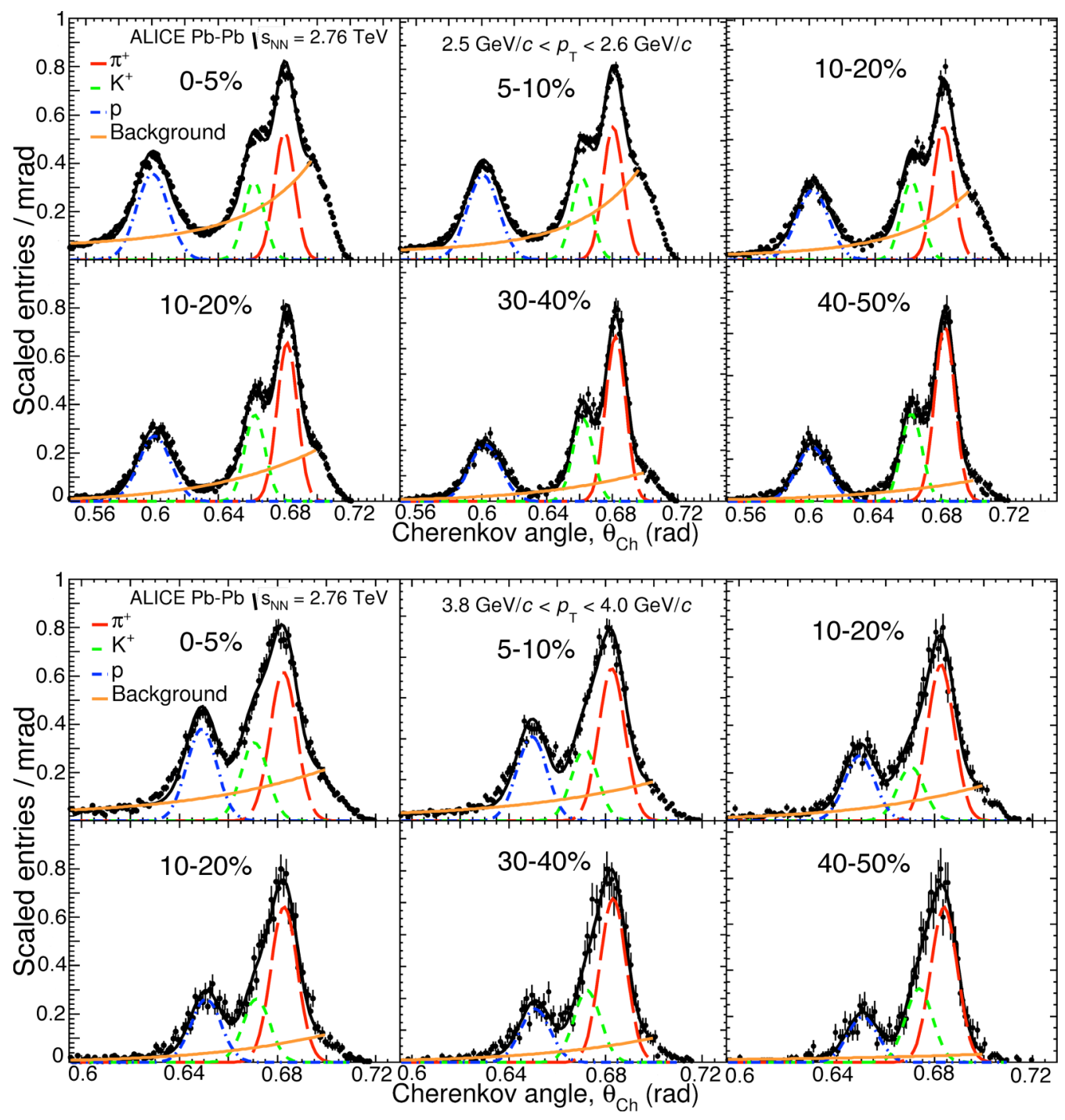

FIG. 17. Distributions of the Cherenkov angle measured in the HMPID for positive tracks having $p_{\mathrm{T}}$ in the range $2.6-2.7 \mathrm{GeV} / c$ (top) and in the range 3.8-4.0 GeV/c (bottom), for six different centrality classes: $0 \%-5 \%, 5 \%-10 \%, 10 \%-20 \%, 20 \%-30 \%, 30 \%-40 \%$, and $40 \%-50 \%$. The histograms have been scaled to have a similar maximum value. The shoulder in the distributions starting at 0.7 rad is a boundary effect owing to the finite chamber geometrical acceptance.

centrality classes. In Fig. 19, a comparison is shown between the mean values of the Cherenkov angle obtained from the fitting procedure with those obtained using a clean sample of protons and pions identified from $\Lambda$ and $K_{S}^{0}$ decays.

To correct for the incorrectly assigned Cherenkov rings, a PID efficiency is used. This efficiency has to be derived from a data set containing identified particles of a single species, so one can use MC or $V^{0}$ daughters. For such a clean set of particles that passes the distance cut, e.g., MC pions as in Fig. 16, the PID efficiency is

$$
\varepsilon_{\mathrm{PID}}=\frac{N(\text { signal })}{N(\text { signal and background })},
$$

where the signal is the integral of the Gaussian fit function. The PID efficiency has been evaluated from MC simulations that reproduce the background observed in the data well. A data-driven cross-check of the efficiency has been performed using a clean sample of $V^{0}$ daughter tracks. The comparison between data and $\mathrm{MC}$ is shown in Fig. 20 for $0 \%-5 \%$ and 40\%-50\% centrality classes and shows good agreement. We also observe that, as expected, the efficiency decreases for more central collisions owing to the occupancy effects mentioned above. The maximum value of the PID efficiency is $\approx 80 \%$ at $p_{\mathrm{T}} \sim 6 \mathrm{GeV} / c$ in the $40 \%-50 \%$ centrality class. As an additional check of the PID efficiency, the ratio between the raw yields extracted from the fit (signal) corrected by the PID efficiency and the total entries in the original histogram (signal and background) has been evaluated for each $p_{\mathrm{T}}$ bin for all centralities. The ratio is consistent with unity when the systematic uncertainties listed in Table III are taken into account.

The systematic uncertainty for the HMPID analysis has contributions from tracking and PID. These uncertainties have been estimated by individually changing the track selection cuts and the parameters of the fit function used to extract the raw yields. The means of the Gaussian functions have been changed by $\pm \sigma$. Similarly, the widths of the Gaussian 

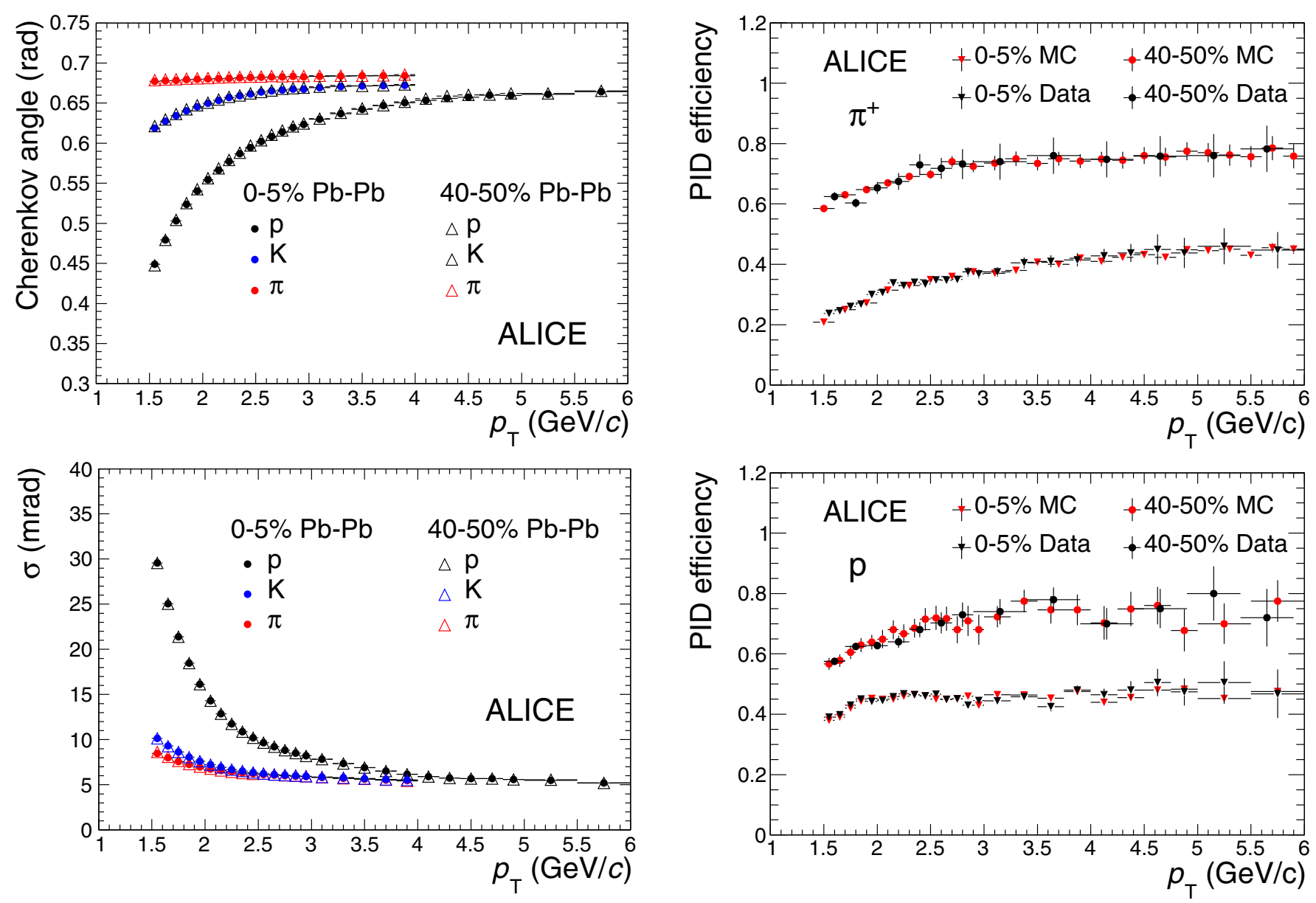

FIG. 18. Mean Cherenkov angle (top) and standard deviation (bottom) values for pions, kaons, and protons obtained by the three-Gaussian fitting procedure as a function of $p_{\mathrm{T}}$ for $0 \%-5 \%$ and $40 \%-50 \%$ centrality $\mathrm{Pb}-\mathrm{Pb}$ collisions. The data points from the two different centrality classes overlap such that the difference is smaller than the size of the symbols used.

functions have been varied by $\pm 10 \%$, accounting for the maximum expected variation of the resolution as a result of the different running conditions of the detector during data acquisition that can have an impact on the performance. When the means are changed, the widths are fixed to the default

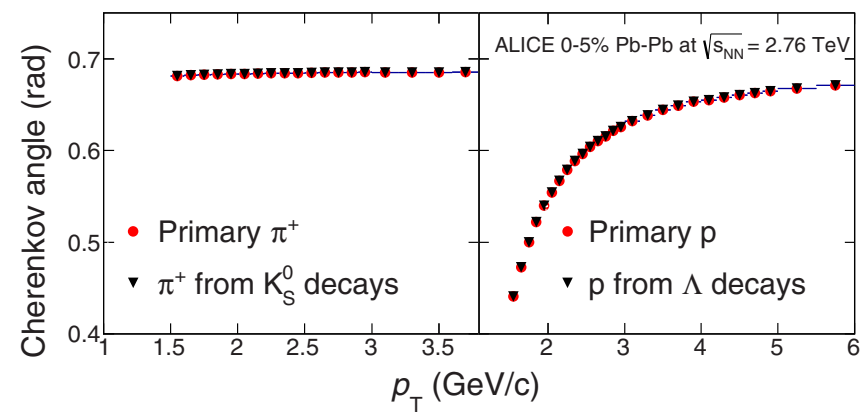

FIG. 19. Comparison of the mean Cherenkov angle values obtained by the three-Gaussian fitting procedure and those evaluated from the $\mathrm{V}^{0} \mathrm{~s}$ study for pions (left) and protons (right) in the most central $\mathrm{Pb}-\mathrm{Pb}$ collisions.

FIG. 20. Identification efficiency for pions (top) and protons (bottom) selected exploiting $\mathrm{V}^{0}$ decay properties, compared with the MC results for primary tracks for $0 \%-5 \%$ and $40 \%-50 \%$ centrality classes.

value, and vice versa. The parameter variation is performed for all three particles species. In addition, the uncertainty on the association of the track to the matched cluster is obtained by varying the value of the distance cut required for the match by $\pm 1 \mathrm{~cm}$. These contributions do not vary with the collision centrality. To estimate the uncertainty owing to the incomplete knowledge of the shape of the background distribution, an

TABLE III. Main sources of systematic uncertainties for the HMPID $\mathrm{Pb}-\mathrm{Pb}$ analysis.

\begin{tabular}{|c|c|c|c|c|c|c|}
\hline \multirow{2}{*}{$\begin{array}{l}\text { Effect } \\
p_{\mathrm{T}} \text { range }(\mathrm{GeV} / c)\end{array}$} & \multicolumn{2}{|c|}{$\pi^{ \pm}$} & \multicolumn{2}{|c|}{$K^{ \pm}$} & \multicolumn{2}{|c|}{$p$ and $\bar{p}$} \\
\hline & 2.5 & 4 & 2.5 & 4 & 2.5 & 4 \\
\hline PID $(\%)$ & 6 & 12 & 6 & 12 & 4 & 5 \\
\hline Tracking efficiency $(\%)$ & \multicolumn{2}{|c|}{6} & \multicolumn{2}{|c|}{6} & & \\
\hline Distance cut correction $(\%)$ & 6 & 2 & 6 & 2 & 4 & 2 \\
\hline Background (Pb-Pb 0\%-5\%) (\%) & 10 & 4 & 5 & 3 & 5 & \\
\hline Background (Pb-Pb 5\%-10\%) (\%) & 7 & 4 & 3 & 2 & 3 & \\
\hline Background (Pb-Pb 10\%-20\%) (\%) & 6 & 4 & 3 & 2 & 3 & \\
\hline Background ( $\mathrm{Pb}-\mathrm{Pb} 20 \%-30 \%)(\%)$ & 5 & 3 & 3 & 2 & 2 & \\
\hline Background (Pb-Pb 30\%-40\%) (\%) & 3 & 1 & 2 & 1 & 2 & \\
\hline Background (Pb-Pb 40\%-50\%) (\%) & 2 & 1 & 2 & 1 & 2 & 1 \\
\hline
\end{tabular}


TABLE IV. The $p_{\mathrm{T}}$ ranges $(\mathrm{GeV} / c)$ used in the combination of the most central results. In $p p$ and peripheral $\mathrm{Pb}-\mathrm{Pb}$ collisions the separation power is different and in some cases the $p_{\mathrm{T}}$ ranges therefore change a little.

\begin{tabular}{lccc}
\hline \hline & ITS + TPC + TOF & HMPID & TPC $d E / d x$ rel. rise \\
\hline$\pi^{ \pm}$ & $0.1-3.0$ & $1.5-4.0$ & $2.0-20.0$ \\
$K^{ \pm}$ & $0.2-3.0$ & $1.5-4.0$ & $3.0-20.0$ \\
$p(\bar{p})$ & $0.3-4.6$ & $1.5-6.0$ & $3.0-20.0$ \\
$K / \pi$ & $0.2-3.0$ & $1.5-4.0$ & $3.0-20.0$ \\
$p / \pi$ & $0.3-3.0$ & $1.5-4.0$ & $3.0-20.0$ \\
\hline \hline
\end{tabular}

alternative background function, depending on $\tan (\theta)$ and derived from geometrical considerations in case of orthogonal tracks [55], has been used,

$$
f(\theta)=a+b \times \tan \theta+c \times\left[\tan \theta\left(1+\tan ^{2} \theta\right)\right]^{d},
$$

where $a, b, c$, and $d$ are free parameters. The corresponding systematic uncertainty reaches a maximum value at low momenta for the most central collisions $(\approx 15 \%$ for pions and $\approx 8 \%$ for kaons and protons). The systematic uncertainty decreases with $p_{\mathrm{T}}$ because, as previously explained, the background contribution decreases with increasing track momentum. A summary of the different contributions to the systematic uncertainty for the HMPID Pb-Pb analysis is given in Table III.

\section{RESULTS AND DISCUSSION}

The measurement of charged pion, kaon, and (anti)proton transverse momentum spectra has been performed via several independent analyses, each one focusing on a subrange of the total $p_{\mathrm{T}}$ distribution, using individual detectors and specific techniques to optimize the signal extraction (see Table IV). The results were combined in the overlapping ranges using a weighted average with the independent systematic uncertainties as weights (a 3\% common systematic uncertainty owing to the TPC tracking is added directly to the combined spectrum). The statistical uncertainties are much smaller and therefore neglected in the combination weights. For $p_{\mathrm{T}}>4 \mathrm{GeV} / c$ only the TPC $d E / d x$ relativistic rise analysis is used for all species. Figure 21 shows the ratio of individual spectra to the combined spectrum for the $0 \%-5 \%, 20 \%-40 \%$, and $60 \%-80 \%$ central $\mathrm{Pb}-\mathrm{Pb}$ data, illustrating the compatibility between the different analyses. In the centrality intervals where the HMPID measurements are available, they improve the systematic uncertainty of the kaon and proton yields by approximately a factor of two in the $p_{\mathrm{T}}$ region where it is later observed that the peaks of the kaon-to-pion and the proton-to-pion ratios are located (see Figs. 25 and 26). We note that the final charged-pion spectra are consistent with the neutral-pion spectra scaled by a factor of two within statistical and systematic uncertainties [57].

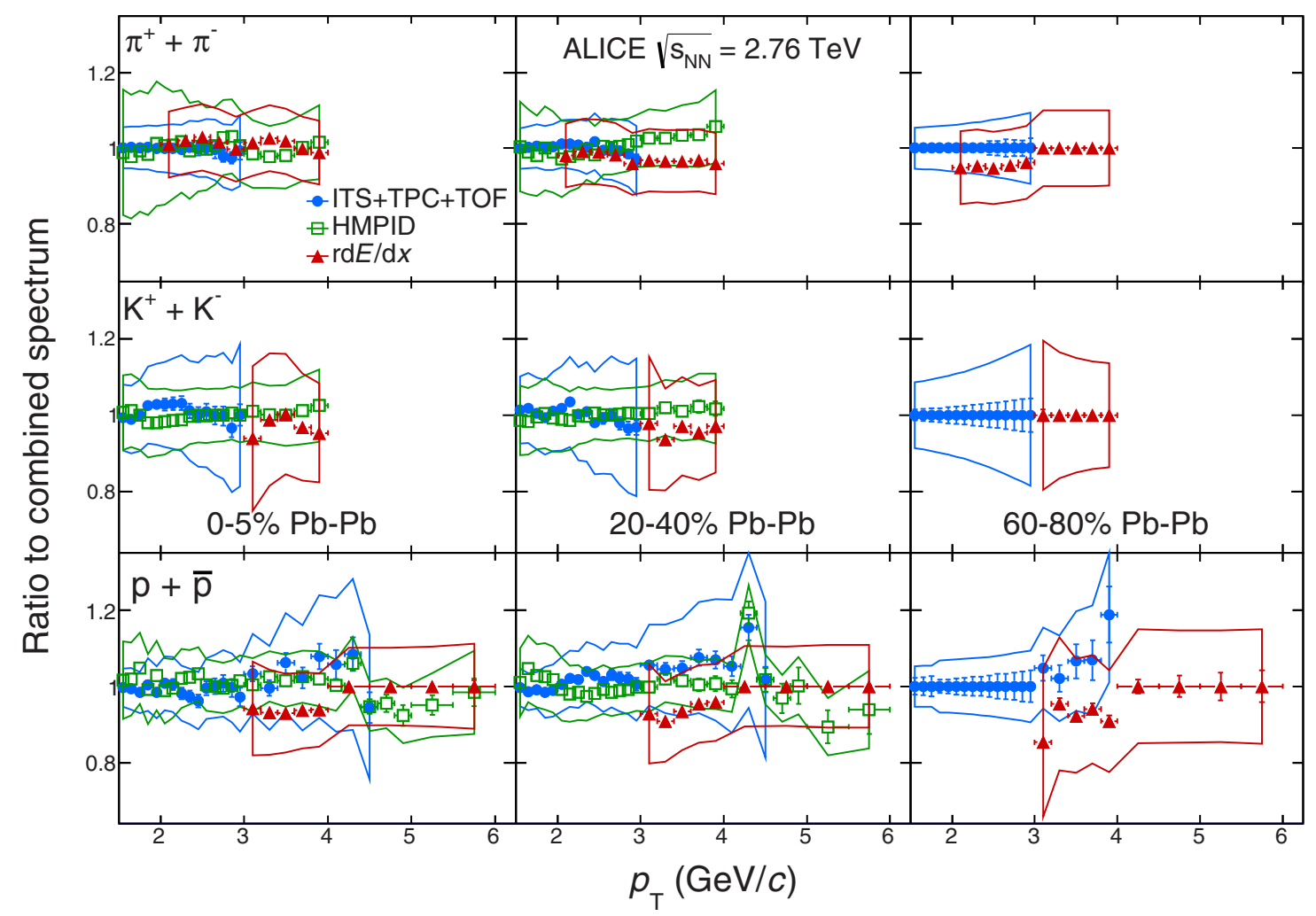

FIG. 21. The ratio of individual spectra to the combined spectrum as a function of $p_{\mathrm{T}}$ for pions (top row), kaons (middle row), and protons (bottom row). From left to right the columns show $0 \%-5 \%, 20 \%-40 \%$, and $60 \%-80 \%$ (where there are no HMPID results). Only the $p_{\mathrm{T}}$ range where the analyses overlap is shown. For $p_{\mathrm{T}}>4 \mathrm{GeV} / c$, no combination is done and the TPC $d E / d x$ relativistic rise results are used directly, which gives rise to a small discontinuity for protons at this $p_{\mathrm{T}}$. The ITS + TPC + TOF spectra are the results published in Ref. [44]. The statistical and independent systematic uncertainties are shown as vertical error bars and as a band, respectively, and only include those on the individual spectra. 


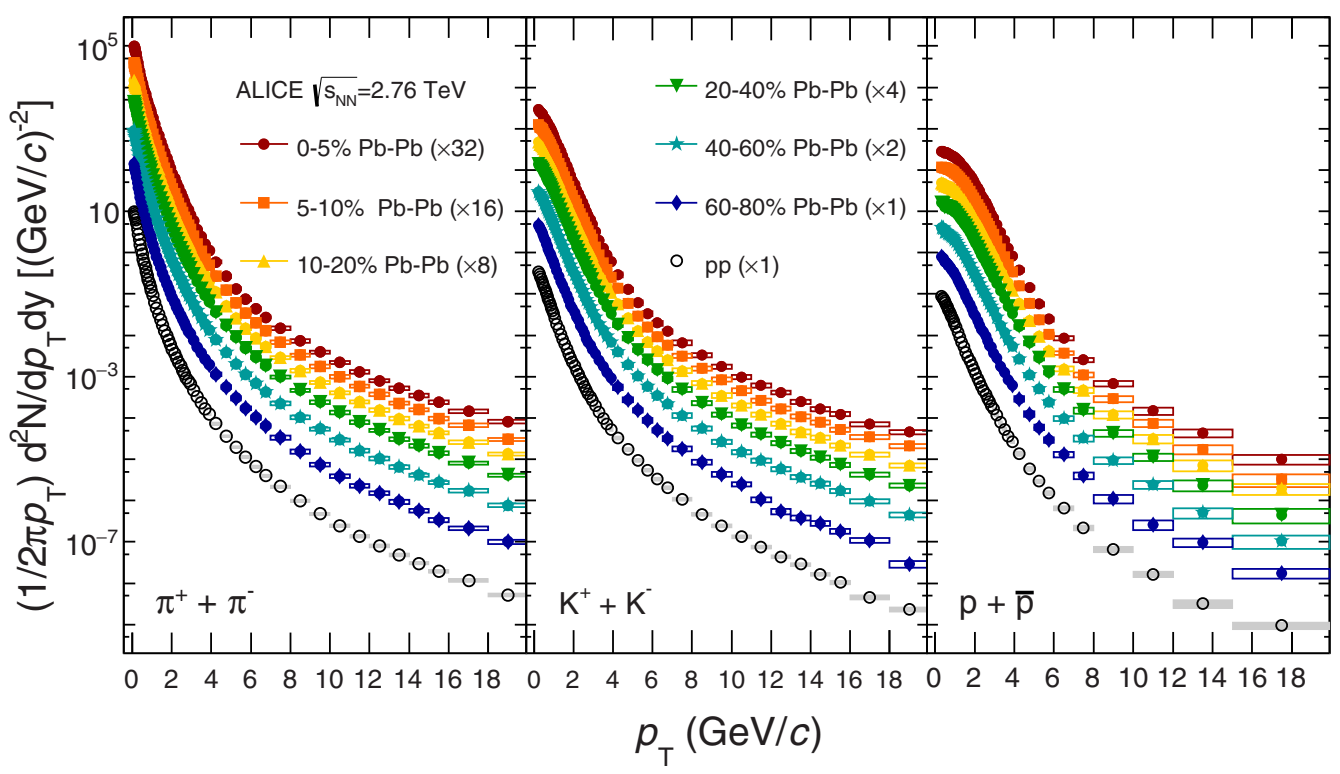

FIG. 22. Transverse momentum spectra of charged pions (left), kaons (middle), and (anti)protons (right) measured in $\mathrm{Pb}-\mathrm{Pb}$ and $p p$ collisions at $\sqrt{s_{N N}}=2.76 \mathrm{TeV}$. The systematic and statistical error are plotted as color boxes and vertical error bars (hard to see), respectively. The spectra have been scaled by the factors listed in the legend for clarity.

The final combined transverse momentum distributions for the three particle species are shown in Fig. 22. For $p_{\mathrm{T}}<3 \mathrm{GeV} / c$, a hardening of the spectra is observed going from peripheral to central events. This effect is mass dependent and is characteristic of hydrodynamic flow, as discussed in Ref. [44]. For high $p_{\mathrm{T}}(>10 \mathrm{GeV} / c)$ the spectra follow a power-law shape, as expected from perturbative QCD (pQCD) calculations. In the following, the high- $p_{\mathrm{T}}$ results are first discussed before going on to the intermediate $p_{\mathrm{T}}$ region.

\section{A. The high- $p_{\mathrm{T}}$ results}

To study jet quenching at high $p_{\mathrm{T}}$, the nuclear modification factor, $R_{A A}$, is constructed. The $R_{A A}$ is

$$
R_{A A}=\frac{d^{2} N_{\mathrm{id}}^{A A} / d y d p_{\mathrm{T}}}{\left\langle T_{A A}\right\rangle d^{2} \sigma_{\mathrm{id}}^{\mathrm{pp}} / d y d p_{\mathrm{T}}},
$$

where $N_{\mathrm{id}}^{A A}$ and $\sigma_{\mathrm{id}}^{p p}$ are the charged-particle yield in nucleusnucleus $(A-A)$ collisions and the cross section in $p p$ collisions,

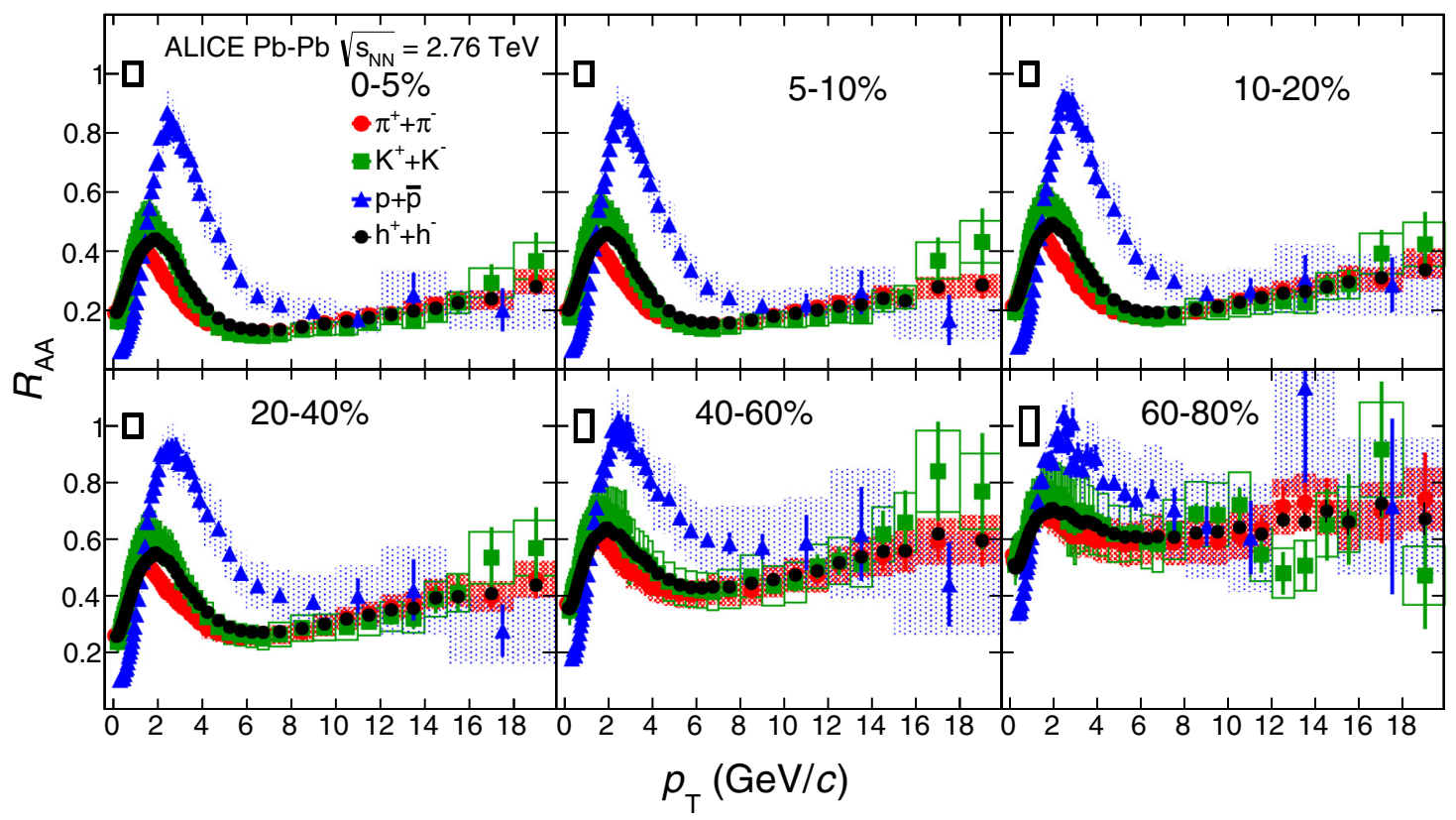

FIG. 23. The nuclear modification factor $R_{A A}$ as a function of $p_{\mathrm{T}}$ for different particle species. Results for different collision centralities are shown. Statistical and PID systematic uncertainties are plotted as vertical error bars and boxes around the points, respectively. The total normalization uncertainty ( $p p$ and $\mathrm{Pb}-\mathrm{Pb}$ ) is indicated in each panel by the vertical scale of the box centered at $p_{\mathrm{T}}=1 \mathrm{GeV} / c$ and $R_{A A}=1[16]$. 


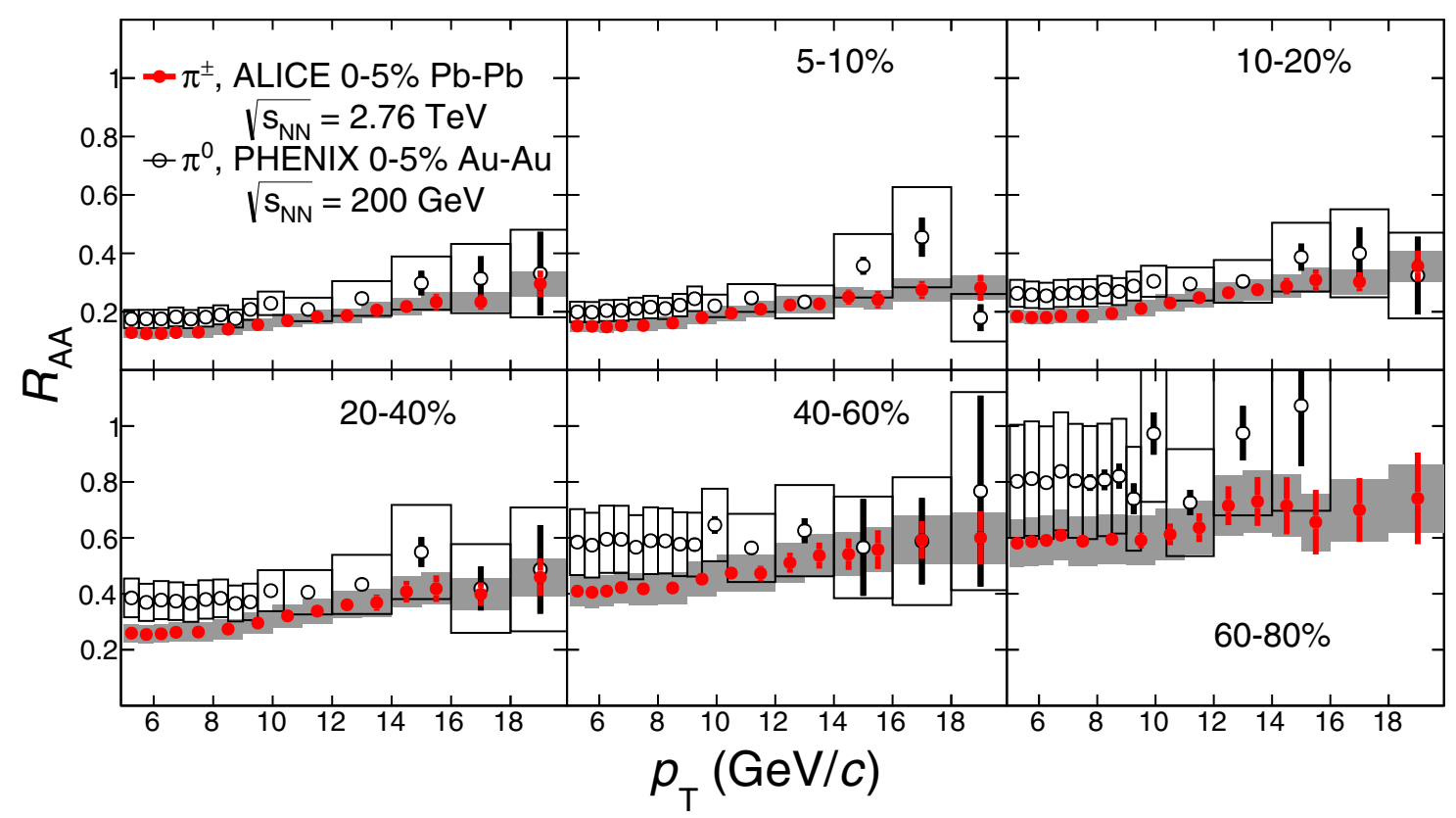

FIG. 24. The nuclear modification factor $R_{A A}$ as a function of $p_{\mathrm{T}}$ for charged pions, compared with PHENIX results for neutral pions [65]. Results for different collision centralities are shown. Statistical and PID systematic uncertainties are plotted as vertical error bars and boxes around the points, respectively.

respectively, and $\left\langle T_{A A}\right\rangle$ is the nuclear overlap function. The latter is obtained from a Glauber model [58] and is related to the average number of binary nucleon-nucleon collisions $\left(N_{\text {coll }}\right)$ and the inelastic nucleon-nucleon cross section as $\left\langle T_{A A}\right\rangle=$ $\left\langle N_{\text {coll }}\right\rangle / \sigma_{\text {inel }}^{N N}$.

Figure 23 shows the $R_{A A}$ for all centrality classes. The results show that for all centrality classes any particle species dependence of the nuclear modification for $p_{\mathrm{T}}>10 \mathrm{GeV} / c$ is small, compared with the large suppression $\left(R_{A A} \ll 1\right)$. This suggests that jet quenching does not produce signatures that affect the particle species composition for the leading particles. The results presented in this paper are all done at the particle level, while for some models that motivated these studies the predictions are done for jets, e.g., the Sapeta-Wiedemann model [22]. It is not obvious how to compare the results presented here with such calculations. In the following we therefore discuss how inclusive $p_{\mathrm{T}}$ spectra compare with inclusive jet $p_{\mathrm{T}}$ spectra. In particular, it is examined if the results are likely to be affected by a quenched jet fragmentation bias (if quenched jets emit less high- $p_{\mathrm{T}}$ particles than unquenched ones) or a surface bias (if unquenched jets from the surface dominate).

At the LHC, by studying dijets in $\mathrm{Pb}-\mathrm{Pb}$ collisions and selecting on the dijet asymmetry, one can study samples with large asymmetries where one knows, based on comparisons with $p p$ results, that at least the subleading jet has suffered a large energy loss [8,9]. The study of the fragmentation functions (FFs) for these quenched jets has shown that for charged tracks with $p_{\mathrm{T}}>4 \mathrm{GeV} / c$ they are similar to those observed in $p p$ collisions for subleading jets with $p_{\mathrm{T}, \mathrm{jet}}>$ $50 \mathrm{GeV} / c$ [59], in agreement with what one also finds for inclusive jets [60]. This rules out a large fragmentation bias (for lower jet $p_{\mathrm{T}}$ see below) and suggests that any surface bias is the same as for inclusive jets. To understand the jet $p_{\mathrm{T}}$ covered by the results presented here, one can now, thanks to the similarity of the FFs in $p p$ and $\mathrm{Pb}-\mathrm{Pb}$ collisions, rely on NLO pQCD calculations for $p p$ collisions. The FFs found to best describe the inclusive charged particle spectra [61] are the Kretzer distributions [62]. NLO pQCD calculations using the Kretzer FFs suggest that more than half of the particles with $p_{\mathrm{T}}$ between 10 and $20 \mathrm{GeV} / c$ are from gluon jets and that the typical jet $p_{\mathrm{T}}$ is roughly a factor of 2-3 larger than the hadron $p_{\mathrm{T}}\left(\langle z\rangle=p_{\mathrm{T}, \text { hadron }} / p_{\mathrm{T}, \mathrm{jet}} \approx 0.4\right)$ [61]. ${ }^{5}$ The conclusions for jets with $p_{\mathrm{T}, \text { jet }}>50 \mathrm{GeV} / c$ are therefore expected to be directly applicable also for the highest- $p_{\mathrm{T}}$ particles studied here. ALICE has studied charged jets in $\mathrm{Pb}-\mathrm{Pb}$ collisions where it was found that requiring a minimum one track with $p_{\mathrm{T}}>10 \mathrm{GeV} / c$ in a jet gives the same fragmentation bias of the jet reconstruction efficiency in $\mathrm{Pb}-\mathrm{Pb}$ collisions as in PYTHIA for $20<p_{\mathrm{T} \text {,ch. jet }}<110 \mathrm{GeV} / c$ [63], so there is no evidence even for lower $p_{\mathrm{T}}$ jets that there is a different fragmentation bias in $\mathrm{Pb}-\mathrm{Pb}$ collisions than in $p p$ collisions. The results in Fig. 23 therefore indicate that for jets with final $p_{\mathrm{T}}$ of order 25 to $50 \mathrm{GeV} / c$, jet quenching does not produce large particle-species-dependent effects in the hard core of the jet where leading particle production mainly occurs.

To be able to set stronger constraints, one needs theoretical modeling. As the $R_{A A}$ for charged pions, kaons, and protons reported here for $p_{\mathrm{T}}>10 \mathrm{GeV} / c$ are all compatible to the $R_{A A}$ for inclusive charged particles [16] and neutral pions [57],

\footnotetext{
${ }^{5}$ The publication contains only calculations for $\sqrt{s}=900 \mathrm{GeV}$ and $\sqrt{s}=7 \mathrm{TeV}$ that have been averaged as an approximate estimate for the energy of $\sqrt{s}=2.76 \mathrm{TeV}$ shown here because the energy dependence is not that strong.
} 

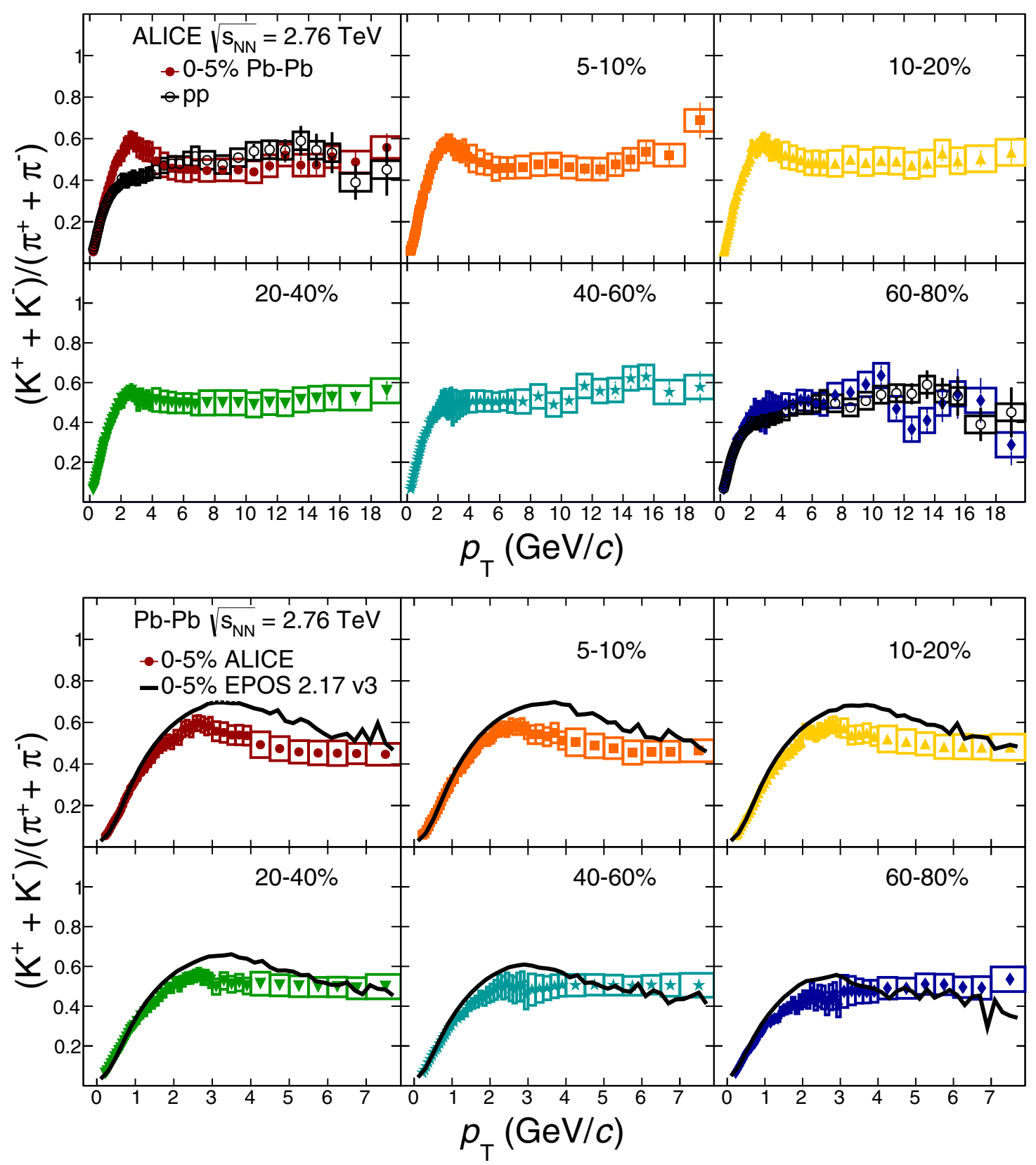

FIG. 25. Charged-kaon to charged-pion ratio as a function of transverse momentum (solid markers). The top panels show the full $p p$ with the $p p$ results (open markers) overlaid in the most central and the most peripheral centrality class. In the bottom panels the $\mathrm{Pb}-\mathrm{Pb}$ results for $p_{\mathrm{T}}<8 \mathrm{GeV} / c$ are compared with EPOS model 2.17-3 (line). The systematic and statistical error are plotted as color boxes and vertical error bars, respectively.

we refer to these papers for comparisons with models without large particle-species-dependent effects. When compared with models which include large particle-species-dependent effects, the results indicate that the jet-quenching mechanism does not involve direct exchange of quantum numbers with the medium, and there are also no indications of a modified color structure of the fragmentation [22] or that the probe is excited to other color states [23]. Models in which the hadronization of jet fragments occurs in the medium also appear to be ruled out [24]. It seems that the medium quenches the jet as a whole rather than directly interacting with its fragments. Such a picture has recently been proposed [64], arguing that the medium typically cannot resolve the structure inside the hard core of the jet such that all fragments lose energy coherently.

In Fig. 24, the $R_{A A}$ for charged pions, the most precise measurement in this work and the one least sensitive to radial flow, is compared with the $R_{A A}$ for neutral pions measured by PHENIX [65] at the RHIC. ${ }^{6}$ The ALICE results are systematically below the PHENIX values for $p_{\mathrm{T}}<10 \mathrm{GeV} / c$

\footnotetext{
${ }^{6}$ The results have been obtained from the tables at the PHENIX web site and the 5\%-10\% data set has been constructed from the $0 \%-5 \%$ and $0 \%-10 \%$ data sets.
} 

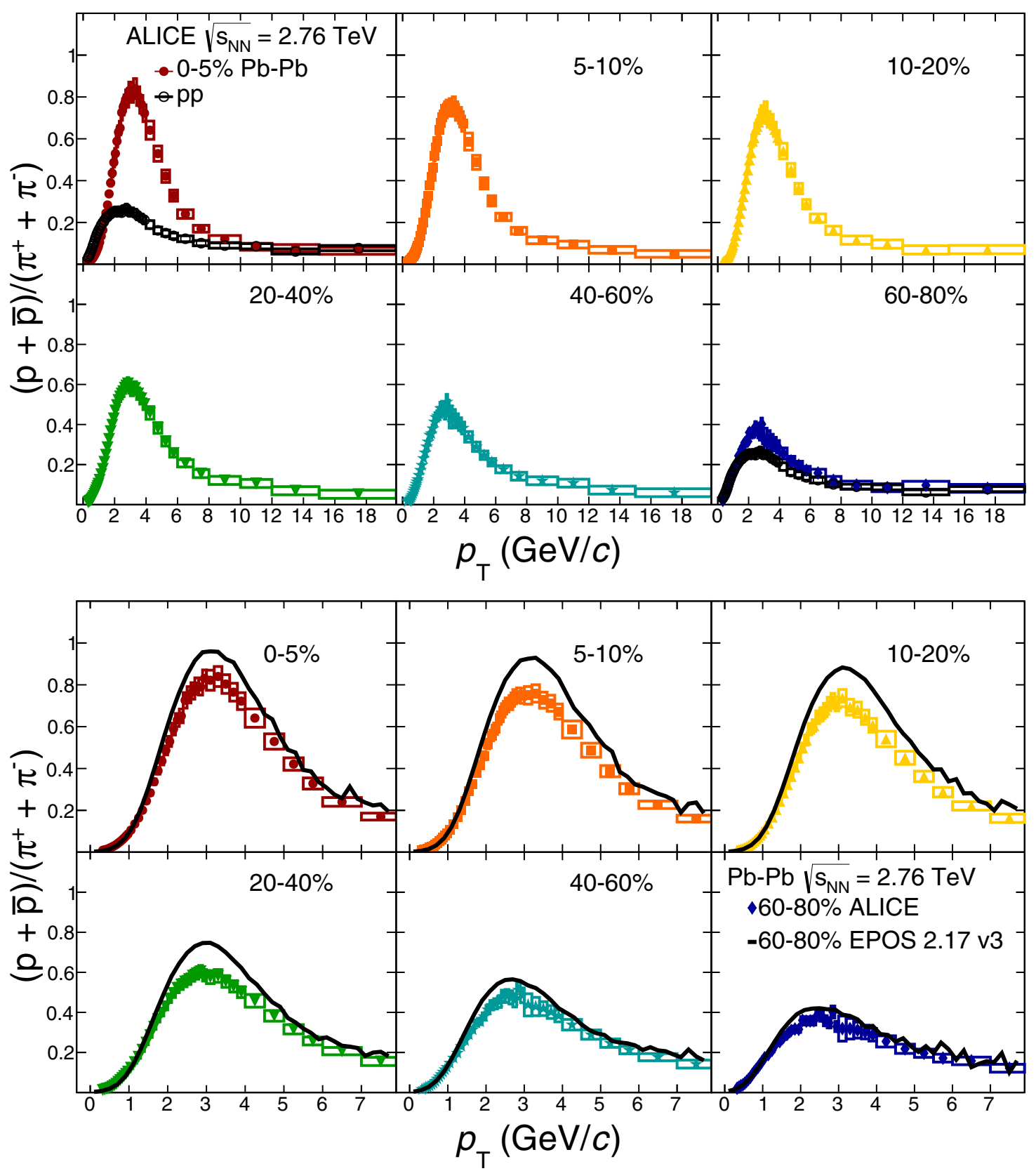

FIG. 26. (Anti)proton to charged pion ratio as a function of transverse momentum (solid markers). The top panels show the full $p p$ with the $p p$ results (open markers) overlaid in the most central and the most peripheral centrality class. In the bottom panels the $\mathrm{Pb}-\mathrm{Pb}$ results for $p_{\mathrm{T}}<8 \mathrm{GeV} / c$ are compared with EPOS model 2.17-3 (line). The systematic and statistical error are plotted as color boxes and vertical error bars, respectively.

but consistent within systematic uncertainties for larger $p_{\mathrm{T}}$. We note that the relative centrality evolution is similar at the two center-of-mass energies. In Ref. [66], a simple study of the $R_{A A}$ at $p_{\mathrm{T}}=10 \mathrm{GeV} / c$ found that the energy loss is $\approx 40 \%$ larger at the LHC than at the RHIC in all centrality classes (it scales as $\sqrt{d N / d \eta}$ for a fixed initial geometry).

The proton-to-pion and the kaon-to-pion ratios as a function of $p_{\mathrm{T}}$ are shown in Figs. 25 and 26. The similarity at high $p_{\mathrm{T}}$ for the $R_{A A}$ implies that the particle ratios there are also the same in $p p$ and $\mathrm{Pb}-\mathrm{Pb}$ collisions. Because the particle ratios are independent of $p_{\mathrm{T}}$ in this region, we use the integrated particle ratios for $p_{\mathrm{T}}>10 \mathrm{GeV} / c$ to elucidate the precision with which the suppression of pions, kaons, and protons is similar; see Fig. 27. The advantage of using particle ratios is that the results for heavy-ion collisions can be shown separately from the $p p$ results. Furthermore, in the ratios the systematic uncertainty associated with the inclusive charged-particle $p_{\mathrm{T}}$ spectra normalization cancels. All the steps in the high- $p_{\mathrm{T}}$ $d E / d x$ analysis discussed in Sec. II A are done independently for each centrality class (using disjunct data sets) so one does not expect any direct correlations of the results. We conclude that all kaon-to-pion (proton-to-pion) ratios as a function 


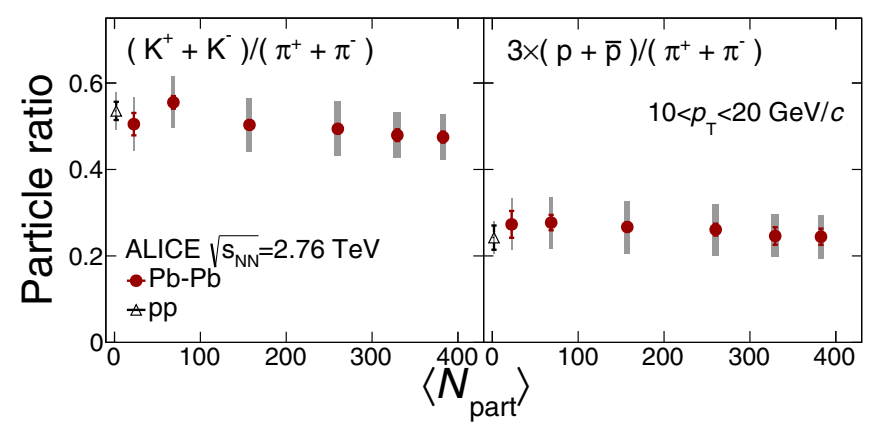

FIG. 27. The integrated particle ratios for $p_{\mathrm{T}}>10 \mathrm{GeV} / c$ in $p p$ and $\mathrm{Pb}-\mathrm{Pb}$ collisions as a function of the number of participants. (Left) The kaon-to-pion ratio. (Right) The proton-to-pion ratio scaled by a factor of 3 for clarity. Statistical and PID systematic uncertainties are plotted as vertical error bars and boxes around the points, respectively. Note that this kaon-to-pion (proton-to-pion) "high- $p_{\mathrm{T}}$ " ratio is $\approx 4$ $(\approx 2)$ times larger than the bulk ratio [44].

of $N_{\text {part }}$ are consistent within the systematic uncertainty of $\approx 10 \%(\approx 20 \%)$. Measurements with improved precision using Run 2 and Run 3 LHC data could reveal possible subtle particle-species differences.

\section{B. The intermediate $p_{\mathrm{T}}$ results}

In the following, the intermediate $p_{\mathrm{T}}$ regions in Figs. 25 and 26, where the proton-to-pion and the kaon-to-pion ratios are enhanced, are discussed.

The observation of the large proton-to-pion ratio at intermediate $p_{\mathrm{T}}$ at the RHIC generated numerous speculations that the degrees of freedom in the medium are constituent quarklike and that they recombine when hadronizing to give rise to distinct meson and baryon properties. As the $\phi$ meson has a mass similar to that of a proton, it is crucial in testing these ideas and results at the RHIC indeed seemed to confirm this picture [67], while at LHC the picture appears to be more complicated $[68,69]$. Some of the models developed to describe results at the RHIC have been extended to the LHC energies. One can, in general, separate recombination models into two classes. In soft models, recombination only occurs for soft thermal radially flowing partons. In Ref. [68] ALICE showed calculations for such a model [31] and the prediction is that at the LHC energies the particle ratios in central collisions are similar to those measured at the highest RHIC energy. In hard recombination models, jet fragments can recombine with both partons from the medium and other jets. At LHC energies, the minijet activity is much larger than at RHIC energies, which motivated predictions for central collisions of particle ratios an order of magnitude larger $(p / \pi \sim 10-20)$ than the peak values reported here and persisting out to much higher $p_{\mathrm{T}}$ [34]. The failure of hard recombination is in qualitative agreement with the picture where the jet interacts with the medium as a whole so that the hard fragments of the jet cannot recombine with partons in the medium or in another jet.

EPOS [70] is a full MC generator which contains both soft and hard physics. It incorporates a hydrodynamical phase and additional hadronization processes at intermediate $p_{\mathrm{T}}$ where the interaction between bulk matter and quenched jets is

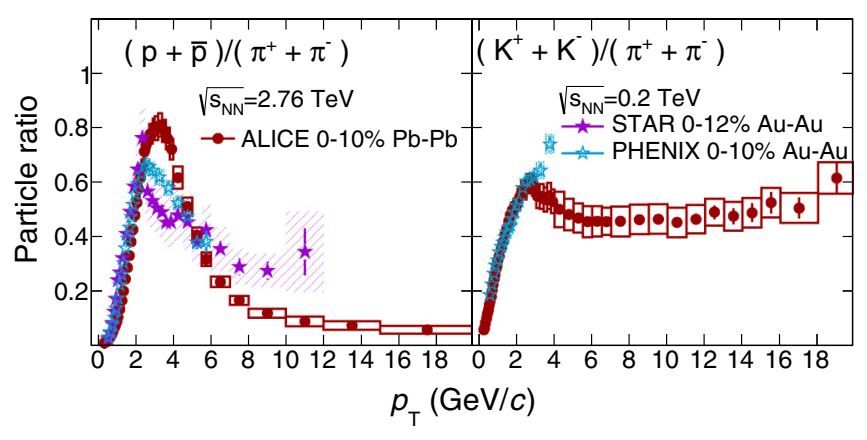

FIG. 28. ALICE (circles) results from $\sqrt{s_{N N}}=2.76 \mathrm{TeV} \mathrm{Pb}-\mathrm{Pb}$ collisions compared with STAR and PHENIX results for $\sqrt{s_{N N}}=$ $200 \mathrm{GeV} \mathrm{Au}$-Au collisions. (Left) The proton-to-pion ratio. (Right) The kaon-to-pion ratio.

considered [71]. This interaction introduces a baryon-meson effect, where fully quenched jets are allowed to hadronize with flowing medium quarks. When we study the full set of ratios at all centralities (Figs. 25 and 26) EPOS generally reproduces the centrality dependence well, even for very peripheral events, where it is known that pure hydrodynamical calculations fail to describe the data [44]. However, EPOS overpredicts the magnitude of both the proton-to-pion and the kaon-to-pion peak; it is therefore critical to understand how important the additional hadronization processes are, relative to the hydrodynamic flow, when all parameters have been tuned.

Figure 28 shows a comparison of particle ratios with results from STAR [25] and PHENIX [27] at the RHIC measured in Au-Au collision at $\sqrt{s_{N N}}=200 \mathrm{GeV}$. In both cases, the results have been averaged for both charge signs for pions and protons. We use the STAR feed-down-corrected data for this comparison. ${ }^{7}$ The proton-to-pion peak at the LHC is approximately $20 \%$ larger than at the RHIC, which is consistent with an increased average radial flow velocity. At high $p_{\mathrm{T}}$, the systematic uncertainties of the STAR data are very large and it was noted in a later publication that they might even be underestimated [26]. Interestingly, there is no evidence for a peak in the kaon-to-pion ratio measured by PHENIX, which is similar to the ALICE data points for $p_{\mathrm{T}} \leqslant 3 \mathrm{GeV} / c$, but continues to rise in the few data points above this $p_{\mathrm{T}}$.

Careful modeling of $p_{\mathrm{T}}$ spectra and azimuthal flow is needed to answer the question of whether there are additional hadronization processes such as soft recombination at the LHC. ${ }^{8}$ Because the multiplicity evolution of particle ratios in $p-\mathrm{Pb}$ collisions is similar to what is observed for $\mathrm{Pb}-\mathrm{Pb}$ collisions [37], it would be interesting to include those results in the modeling, in particular, because there is no indication of jet quenching [73], which conceptually simplifies the problem.

\footnotetext{
${ }^{7}$ Values taken from https://drupal.star.bnl.gov/STAR/files/ starpublications/65/data.html for protons and a similar feed-down correction has been assumed for antiprotons.

${ }^{8}$ We note that in a recent preprint it is shown that soft recombination together with pQCD + quenching can give a good description of pion, kaon, and (anti)proton spectra in central heavy-ion collisions at both the RHIC and the LHC for $1.5<p_{\mathrm{T}}<10 \mathrm{GeV} / c$ [72].
} 


\section{CONCLUSION}

We have reported the centrality-dependent measurement of charged pions, kaons, and (anti)protons at large transverse momenta in $\mathrm{Pb}-\mathrm{Pb}$ collisions at the LHC. When combined with previously published data at lower $p_{\mathrm{T}}$, the new results provide a comprehensive data set of pion, kaon, and (anti)proton $p_{\mathrm{T}}$ spectra with unprecedented systematic precision and $p_{\mathrm{T}}$ reach. The spectra are sensitive to physics mechanisms that differentiate between baryons and mesons, strange and nonstrange, or heavy and light hadrons.

At high $p_{\mathrm{T}}\left(p_{\mathrm{T}}>10 \mathrm{GeV} / c\right)$, particle ratios and nuclear modification factors allow the study of effects related to jet quenching. The measurements in this $p_{\mathrm{T}}$ range do not show any difference in the nuclear modification factor for pions, kaons, and protons. A comparison of the present results with jet measurements and theoretical calculations establishes that jet quenching does not introduce large species-dependent modifications for leading particles. Instead, at high $p_{\mathrm{T}}$, for all six centrality classes and the $p p$ data analyzed here, the same kaon-to-pion and proton-to-pion ratios are obtained within a systematic precision of $\approx 10 \%-20 \%$.

At intermediate $p_{\mathrm{T}}$, calculations are needed to determine whether models containing only hydrodynamics and jet quenching can provide a good description across many observables of the available experimental results or if additional processes such as recombination are needed. Because the initial geometry of the collision directly affects both the flow and the energy loss, the centrality dependence presented in this paper is important for constraining both the low- $p_{\mathrm{T}}$ hydrodynamics and the high- $p_{\mathrm{T}}$ jet quenching in the calculations.

The results in this paper, taken together with the wealth of other high $p_{\mathrm{T}}$ and jet results from the LHC, point toward a need for further development of a microscopic QCD-based picture that explains in detail the relation between the jet, the medium, and the energy loss.

\section{ACKNOWLEDGMENTS}

The ALICE Collaboration would like to thank all its engineers and technicians for their invaluable contributions to the construction of the experiment and the CERN accelerator teams for the outstanding performance of the LHC complex. The ALICE Collaboration gratefully acknowledges the resources and support provided by all Grid centres and the Worldwide LHC Computing Grid (WLCG) collaboration. The ALICE Collaboration acknowledges the following funding agencies for their support in building and running the ALICE detector: State Committee of Science, World Federation of Scientists (WFS) and Swiss Fonds Kidagan, Armenia, Conselho Nacional de Desenvolvimento Científico e Tecnológico (CNPq), Financiadora de Estudos e Projetos (FINEP), Fundação de Amparo à Pesquisa do Estado de São Paulo (FAPESP); National Natural Science Foundation of
China (NSFC), the Chinese Ministry of Education (CMOE) and the Ministry of Science and Technology of China (MSTC); Ministry of Education and Youth of the Czech Republic; Danish Natural Science Research Council, the Carlsberg Foundation and the Danish National Research Foundation; The European Research Council under the European Community's Seventh Framework Programme; Helsinki Institute of Physics and the Academy of Finland; French CNRS-IN2P3, the "Region Pays de Loire," "Region Alsace," "Region Auvergne," and CEA, France; German Bundesministerium fur Bildung, Wissenschaft, Forschung und Technologie (BMBF) and the Helmholtz Association; General Secretariat for Research and Technology, Ministry of Development, Greece; Hungarian Orszagos Tudomanyos Kutatasi Alappgrammok (OTKA) and National Office for Research and Technology (NKTH); Department of Atomic Energy and Department of Science and Technology of the Government of India; Istituto Nazionale di Fisica Nucleare (INFN) and Centro Fermi-Museo Storico della Fisica e Centro Studi e Ricerche "Enrico Fermi," Italy; MEXT Grant-in-Aid for Specially Promoted Research, Japan; Joint Institute for Nuclear Research, Dubna; National Research Foundation of Korea (NRF); Consejo Nacional de Cienca y Tecnologia (CONACYT), Direccion General de Asuntos del Personal Academico(DGAPA), México, Amerique Latine Formation academique-European Commission (ALFA-EC) and the EPLANET Program (European Particle Physics Latin American Network); Stichting voor Fundamenteel Onderzoek der Materie (FOM) and the Nederlandse Organisatie voor Wetenschappelijk Onderzoek (NWO), Netherlands; Research Council of Norway (NFR); National Science Centre, Poland; Ministry of National Education/Institute for Atomic Physics and National Council of Scientific Research in Higher Education (CNCSI-UEFISCDI), Romania; Ministry of Education and Science of Russian Federation, Russian Academy of Sciences, Russian Federal Agency of Atomic Energy, Russian Federal Agency for Science and Innovations and The Russian Foundation for Basic Research; Ministry of Education of Slovakia; Department of Science and Technology, South Africa; Centro de Investigaciones Energeticas, Medioambientales y Tecnologicas (CIEMAT), E-Infrastructure shared between Europe and Latin America (EELA), Ministerio de Economía y Competitividad (MINECO) of Spain, Xunta de Galicia (Consellería de Educación), Centro de Aplicaciones Tecnolgicas y Desarrollo Nuclear (CEADEN), Cubaenergía, Cuba, and IAEA (International Atomic Energy Agency); Swedish Research Council (VR) and Knut \& Alice Wallenberg Foundation (KAW); Ukraine Ministry of Education and Science; United Kingdom Science and Technology Facilities Council (STFC); The United States Department of Energy, the United States National Science Foundation, the State of Texas, and the State of Ohio; Ministry of Science, Education and Sports of Croatia and Unity through Knowledge Fund, Croatia; Council of Scientific and Industrial Research (CSIR), New Delhi, India.
[1] I. Arsene et al. (BRAHMS Collaboration), Quark gluon plasma and color glass condensate at RHIC? The Perspective from the BRAHMS experiment, Nucl. Phys. A 757, 1 (2005).

[2] K. Adcox et al. (PHENIX Collaboration), Formation of dense partonic matter in relativistic nucleus-nucleus collisions at
RHIC: Experimental evaluation by the PHENIX collaboration, Nucl. Phys. A 757, 184 (2005).

[3] B. Back, M. Baker, M. Ballintijn, D. Barton, B. Becker et al., The PHOBOS perspective on discoveries at RHIC, Nucl. Phys. A 757, 28 (2005). 
[4] J. Adams et al. (STAR Collaboration), Experimental and theoretical challenges in the search for the quark gluon plasma: The STAR Collaboration's critical assessment of the evidence from RHIC collisions, Nucl. Phys. A 757, 102 (2005).

[5] K. Aamodt et al. (ALICE Collaboration), Elliptic Flow of Charged Particles in $\mathrm{Pb}-\mathrm{Pb}$ Collisions at $\sqrt{s_{N N}}=2.76 \mathrm{TeV}$, Phys. Rev. Lett. 105, 252302 (2010).

[6] K. Aamodt et al. (ALICE Collaboration), Suppression of charged particle production at large transverse momentum in central Pb-Pb collisions at $\sqrt{s_{N N}}=2.76 \mathrm{TeV}$, Phys. Lett. B 696, 30 (2011).

[7] B. Abelev et al. (ALICE Collaboration), $J / \psi$ Suppression at Forward Rapidity in $\mathrm{Pb}-\mathrm{Pb}$ Collisions at $\sqrt{s_{N N}}=2.76 \mathrm{TeV}$, Phys. Rev. Lett. 109, 072301 (2012).

[8] G. Aad et al. (ATLAS Collaboration), Observation of a Centrality-Dependent Dijet Asymmetry in Lead-Lead Collisions at $\sqrt{s_{N N}}=2.77 \mathrm{TeV}$ with the ATLAS Detector at the LHC, Phys. Rev. Lett. 105, 252303 (2010).

[9] S. Chatrchyan et al. (CMS Collaboration), Observation and studies of jet quenching in $\mathrm{PbPb}$ collisions at nucleon-nucleon center-of-mass energy $\sqrt{s_{N N}}=2.76 \mathrm{TeV}$, Phys. Rev. C 84, 024906 (2011).

[10] M. Gyulassy and M. Plumer, Jet quenching in dense matter, Phys. Lett. B 243, 432 (1990).

[11] M. Gyulassy and X.-n. Wang, Multiple collisions and induced gluon Bremsstrahlung in QCD, Nucl. Phys. B 420, 583 (1994).

[12] E. Wang and X.-N. Wang, Jet Tomography of Dense and Nuclear Matter, Phys. Rev. Lett. 89, 162301 (2002).

[13] K. Adcox et al. (PHENIX Collaboration), Suppression of Hadrons with Large Transverse Momentum in Central $\mathrm{Au}+\mathrm{Au}$ Collisions at $\sqrt{s_{N N}}=130-\mathrm{GeV}$, Phys. Rev. Lett. 88, 022301 (2002).

[14] C. Adler et al. (STAR Collaboration), Centrality Dependence of High $p_{T}$ Hadron Suppression in Au+Au Collisions at $\sqrt{s}_{N N}=$ 130-GeV, Phys. Rev. Lett. 89, 202301 (2002).

[15] J. Adams et al. (STAR Collaboration), Transverse Momentum and Collision Energy Dependence of High $\mathrm{p}(\mathrm{T})$ Hadron Suppression in $\mathrm{Au}+\mathrm{Au}$ Collisions at Ultrarelativistic Energies, Phys. Rev. Lett. 91, 172302 (2003).

[16] B. Abelev et al. (ALICE Collaboration), Centrality dependence of charged particle production at large transverse momentum in $\mathrm{Pb}-\mathrm{Pb}$ collisions at $\sqrt{s_{N N}}=2.76 \mathrm{TeV}$, Phys. Lett. B 720, 52 (2013).

[17] S. Chatrchyan et al. (CMS Collaboration), Study of high-pT charged particle suppression in $\mathrm{PbPb}$ compared to pp collisions at $\sqrt{s_{N N}}=2.76 \mathrm{TeV}$, Eur. Phys. J. C 72, 1945 (2012).

[18] G. Aad et al. (ATLAS Collaboration), Measurement of chargedparticle spectra in $\mathrm{Pb}+\mathrm{Pb}$ collisions at $\sqrt{s_{N N}}=2.76 \mathrm{TeV}$ with the ATLAS detector at the LHC, JHEP 09 (2015) 050.

[19] X.-N. Wang, Effect of jet quenching on high $p_{\mathrm{T}}$ hadron spectra in high-energy nuclear collisions, Phys. Rev. C 58, 2321 (1998).

[20] T. Renk and K. J. Eskola, Proton-antiproton suppression in 200A GeV Au-Au collisions, Phys. Rev. C 76, 027901 (2007).

[21] P. Abreu et al. (DELPHI Collaboration), Identified charged particles in quark and gluon jets, Eur. Phys. J. C 17, 207 (2000).

[22] S. Sapeta and U. A. Wiedemann, Jet hadrochemistry as a characteristics of jet quenching, Eur. Phys. J. C 55, 293 (2008).

[23] P. Aurenche and B. Zakharov, Jet color chemistry and anomalous baryon production in AA-collisions, Eur. Phys. J. C 71, 1829 (2011).
[24] R. Bellwied and C. Markert, In-medium hadronization in the deconfined matter at RHIC and LHC, Phys. Lett. B 691, 208 (2010).

[25] B. Abelev et al. (STAR Collaboration), Identified Baryon and Meson Distributions at Large Transverse Momenta from $\mathrm{Au}+\mathrm{Au}$ Collisions at $\sqrt{s_{N N}}=200$, Phys. Rev. Lett. 97, 152301 (2006).

[26] G. Agakishiev et al. (STAR Collaboration), Identified Hadron Compositions in $\mathrm{p}+\mathrm{p}$ and $\mathrm{Au}+\mathrm{Au}$ Collisions at High Transverse Momenta at $\sqrt{s_{N N}}=200 \mathrm{GeV}$, Phys. Rev. Lett. 108, 072302 (2012).

[27] A. Adare et al. (PHENIX Collaboration), Spectra and ratios of identified particles in $\mathrm{Au}+\mathrm{Au}$ and $\mathrm{d}+\mathrm{Au}$ collisions at $\sqrt{s_{N N}}=$ $200 \mathrm{GeV}$, Phys. Rev. C 88, 024906 (2013).

[28] K. Adcox et al. (PHENIX Collaboration), Centrality Dependence of $\pi^{+} / \pi^{-}, \mathrm{K}^{+} / \mathrm{K}^{-}$, p and anti-p production from $\sqrt{s_{N N}}=$ $130 \mathrm{GeV} \mathrm{Au+Au} \mathrm{collisions} \mathrm{at} \mathrm{RHIC,} \mathrm{Phys.} \mathrm{Rev.} \mathrm{Lett.} \mathrm{88,}$ 242301 (2002).

[29] S. Adler et al. (PHENIX Collaboration), Scaling Properties of Proton and Anti-Proton Production in $\sqrt{s_{N N}}=200 \mathrm{GeV} \mathrm{Au}+\mathrm{Au}$ Collisions, Phys. Rev. Lett. 91, 172301 (2003).

[30] J. Adams et al. (STAR Collaboration), Particle Type Dependence of Azimuthal Anisotropy and Nuclear Modification of Particle Production in $\mathrm{Au}+\mathrm{Au}$ Collisions at $\sqrt{s_{N N}}=200 \mathrm{GeV}$, Phys Rev. Lett. 92, 052302 (2004).

[31] R. J. Fries, B. Müller, C. Nonaka, and S. A. Bass, Hadron production in heavy ion collisions: Fragmentation and recombination from a dense parton phase, Phys. Rev. C 68, 044902 (2003).

[32] V. Topor Pop, M. Gyulassy, J. Barrette, C. Gale, X. Wang et al., Baryon junction loops in HIJING / B anti-B v2.0 and the baryon/meson anomaly at RHIC, Phys. Rev. C 70, 064906 (2004).

[33] S. J. Brodsky and A. Sickles, The baryon anomaly: Evidence for color transparency and direct hadron production at RHIC, Phys. Lett. B 668, 111 (2008).

[34] R. C. Hwa and C. Yang, Proton Enhancement at Large $p_{\mathrm{T}}$ at LHC Without Structure in Associated-Particle Distribution, Phys. Rev. Lett. 97, 042301 (2006).

[35] B. Abelev et al. (ALICE Collaboration), Production of charged pions, kaons and protons at large transverse momenta in pp and $\mathrm{Pb}-\mathrm{Pb}$ collisions at $\sqrt{s_{N N}}=2.76 \mathrm{TeV}$, Phys. Lett. B 736, 196 (2014).

[36] B. Abelev et al. (ALICE Collaboration), Long-range angular correlations of $\pi, \mathrm{K}$ and $\mathrm{p}$ in $\mathrm{p}-\mathrm{Pb}$ collisions at $\sqrt{s_{N N}}=5.02 \mathrm{TeV}$, Phys. Lett. B 726, 164 (2013).

[37] B. Abelev et al. (ALICE Collaboration), Multiplicity dependence of pion, kaon, proton and lambda production in $\mathrm{p}$ $\mathrm{Pb}$ collisions at $\sqrt{s_{N N}}=5.02 \mathrm{TeV}$, Phys. Lett. B 728, 25 (2014).

[38] V. Khachatryan et al. (CMS Collaboration), Long-range twoparticle correlations of strange hadrons with charged particles in $\mathrm{pPb}$ and $\mathrm{PbPb}$ collisions at LHC energies, Phys. Lett. B 742, 200 (2015).

[39] E. Shuryak and I. Zahed, High-multiplicity pp and pA collisions: Hydrodynamics at its edge, Phys. Rev. C 88, 044915 (2013).

[40] K. Werner, M. Bleicher, B. Guiot, I. Karpenko, and T. Pierog, Evidence for Flow from Hydrodynamic Simulations of $p-\mathrm{Pb}$ Collisions at 5.02 TeV from $v_{2}$ Mass Splitting, Phys. Rev. Lett. 112, 232301 (2014). 
[41] P. Bozek, W. Broniowski, and G. Torrieri, Mass Hierarchy in Identified Particle Distributions in Proton-Lead Collisions, Phys. Rev. Lett. 111, 172303 (2013).

[42] K. Aamodt et al. (ALICE Collaboration), The ALICE experiment at the CERN LHC, J. Instrum. 3, S08002 (2008).

[43] B. Abelev et al. (ALICE Collaboration), Performance of the ALICE Experiment at the CERN LHC, Int. J. Mod. Phys. A 29, 1430044 (2014).

[44] B. Abelev et al. (ALICE Collaboration), Centrality dependence of $\pi, \mathrm{K}, \mathrm{p}$ production in $\mathrm{Pb}-\mathrm{Pb}$ collisions at $\sqrt{s_{N N}}=2.76 \mathrm{TeV}$, Phys. Rev. C 88, 044910 (2013).

[45] E. Abbas et al. (ALICE Collaboration), Performance of the ALICE VZERO system, J. Instrum. 8, P10016 (2013).

[46] J. Alme, Y. Andres, H. Appelshauser, S. Bablok, N. Bialas et al., The ALICE TPC, a large 3-dimensional tracking device with fast readout for ultra-high multiplicity events, Nucl. Instrum. Methods A 622, 316 (2010).

[47] B. Abelev et al. (ALICE Collaboration), Energy dependence of the transverse momentum distributions of charged particles in pp collisions measured by ALICE, Eur. Phys. J. C 73, 2662 (2013).

[48] H. Bichsel, A method to improve tracking and particle identification in TPCs and silicon detectors, Nucl. Instrum. Methods A 562, 154 (2006).

[49] D. Buskulic et al. (ALEPH Collaboration), Performance of the ALEPH detector at LEP, Nucl. Instrum. Methods A 360, 481 (1995).

[50] P. Christiansen et al., The influence of detector effects on TPC performance, Nucl. Instrum. Methods A 609, 149 (2009).

[51] B. Abelev et al. (ALICE Collaboration), $K_{S}^{0}$ and $\Lambda$ Production in Pb-Pb Collisions at $\sqrt{s_{N N}}=2.76 \mathrm{TeV}$, Phys. Rev. Lett. 111, 222301 (2013).

[52] T. Sjostrand, S. Mrenna, and P. Z. Skands, PYTHIA 6.4 Physics and Manual, J. High Energy Phys. 05 (2006) 026.

[53] F. W. Bopp, R. Engel, and J. Ranft, Rapidity gaps and the PHOJET Monte Carlo, arXiv:hep-ph/9803437 [hep-ph].

[54] X.-N. Wang and M. Gyulassy, Hijing: A monte carlo model for multiple jet production in pp, pa, and aa collisions, Phys. Rev. D 44, 3501 (1991).

[55] ALICE Collaboration, ALICE Technical Design Report of the High Momentum Particle Identification Detector, CERN/LHCC 98-19, ALICE TDR 1 (1998).

[56] D. Cozza, D. Di Bari, D. Elia, E. Nappi, A. Di Mauro et al., Recognition of Cherenkov patterns in high multiplicity environments, Nucl. Instrum. Methods A 482, 226 (2002).

[57] B. Abelev et al. (ALICE Collaboration), Neutral pion production at midrapidity in pp and $\mathrm{Pb}-\mathrm{Pb}$ collisions at $\sqrt{s_{N N}}=2.76 \mathrm{TeV}$, Eur. Phys. J. C 74, 3108 (2014).

[58] B. Abelev et al. (ALICE Collaboration), Centrality determination of $\mathrm{Pb}-\mathrm{Pb}$ collisions at $\sqrt{s_{N N}}=2.76 \mathrm{TeV}$ with ALICE, Phys. Rev. C 88, 044909 (2013).
[59] S. Chatrchyan et al. (CMS Collaboration), Measurement of jet fragmentation into charged particles in $p p$ and $\mathrm{PbPb}$ collisions at $\sqrt{s_{N N}}=2.76 \mathrm{TeV}$, J. High Energy Phys. 10 (2012) 087.

[60] S. Chatrchyan et al. (CMS Collaboration), Measurement of jet fragmentation in $\mathrm{PbPb}$ and pp collisions at $\sqrt{s_{N N}}=2.76 \mathrm{TeV}$, Phys. Rev. C 90, 024908 (2014).

[61] D. d'Enterria, K. J. Eskola, I. Helenius, and H. Paukkunen, Confronting current NLO parton fragmentation functions with inclusive charged-particle spectra at hadron colliders, Nucl. Phys. B 883, 615 (2014).

[62] S. Kretzer, Fragmentation functions from flavor inclusive and flavor tagged e+ e- annihilations, Phys. Rev. D 62, 054001 (2000).

[63] B. Abelev et al. (ALICE Collaboration), Measurement of charged jet suppression in $\mathrm{Pb}-\mathrm{Pb}$ collisions at $\sqrt{s_{N N}}=2.76 \mathrm{TeV}$, J. High Energy Phys. 03 (2014) 013.

[64] J. Casalderrey-Solana, Y. Mehtar-Tani, C. A. Salgado, and K. Tywoniuk, New picture of jet quenching dictated by color coherence, Phys. Lett. B 725, 357 (2013).

[65] A. Adare et al. (PHENIX Collaboration), Neutral pion production with respect to centrality and reaction plane in $\mathrm{Au}+\mathrm{Au}$ collisions at $\sqrt{s_{N N}}=200 \mathrm{GeV}$, Phys. Rev. C 87, 034911 (2013).

[66] P. Christiansen, K. Tywoniuk, and V. Vislavicius, Universal scaling dependence of QCD energy loss from data driven studies, Phys. Rev. C 89, 034912 (2014).

[67] B. Abelev et al. (STAR Collaboration), Partonic Flow and PhiMeson Production in $\mathrm{Au}+\mathrm{Au}$ Collisions at $\sqrt{s_{N N}}=200 \mathrm{GeV}$, Phys. Rev. Lett. 99, 112301 (2007).

[68] B. Abelev et al. (ALICE Collaboration), $K^{*}(892)^{0}$ and $\Phi(1020)$ production in $\mathrm{Pb}-\mathrm{Pb}$ collisions at $\sqrt{s_{N N}}=2.76 \mathrm{TeV}$, Phys. Rev. C 91, 024609 (2015).

[69] B. Abelev et al. (ALICE Collaboration), Elliptic flow of identified hadrons in $\mathrm{Pb}-\mathrm{Pb}$ collisions at $\sqrt{s_{\mathrm{NN}}}=2.76 \mathrm{TeV}$, J. High Energy Phys. 06 (2015) 190.

[70] K. Werner, I. Karpenko, M. Bleicher, T. Pierog, and S. Porteboeuf-Houssais, Jets, bulk matter, and their interaction in heavy ion collisions at several tev, Phys. Rev. C 85, 064907 (2012).

[71] K. Werner, Lambda-To-Kaon Ratio Enhancement in Heavy Ion Collisions at Several TeV, Phys. Rev. Lett. 109, 102301 (2012).

[72] V. Minissale, F. Scardina, and V. Greco, Hadrons from coalescence plus fragmentation in AA collisions at energies available at the BNL Relativistic Heavy Ion Collider to the CERN Large Hadron Collider, Phys. Rev. C 92, 054904 (2015).

[73] B. Abelev et al. (ALICE Collaboration), Transverse Momentum Distribution and Nuclear Modification Factor of Charged Particles in $p$-Pb Collisions at $\sqrt{s_{N N}}=5.02 \mathrm{TeV}$, Phys. Rev. Lett. 110, 082302 (2013).

J. Adam, ${ }^{40}$ D. Adamová, ${ }^{83}$ M. M. Aggarwal, ${ }^{87}$ G. Aglieri Rinella, ${ }^{36}$ M. Agnello, ${ }^{111}$ N. Agrawal, ${ }^{48}$ Z. Ahammed, ${ }^{132}$ S. U. Ahn, ${ }^{68}$ I. Aimo, ${ }^{94,111}$ S. Aiola, ${ }^{137}$ M. Ajaz, ${ }^{16}$ A. Akindinov, ${ }^{58}$ S. N. Alam, ${ }^{132}$ D. Aleksandrov, ${ }^{100}$ B. Alessandro, ${ }^{111}$ D. Alexandre, ${ }^{102}$ R. Alfaro Molina, ${ }^{64}$ A. Alici, ${ }^{105,12}$ A. Alkin, ${ }^{3}$ J. R. M. Almaraz, ${ }^{119}$ J. Alme, ${ }^{38}$ T. Alt, ${ }^{43}$ S. Altinpinar, ${ }^{18}$ I. Altsybeev, ${ }^{131}$ C. Alves Garcia Prado, ${ }^{120}$ C. Andrei, ${ }^{78}$ A. Andronic, ${ }^{97}$ V. Anguelov, ${ }^{93}$ J. Anielski, ${ }^{54}$ T. Antičić,${ }^{98}$ F. Antinori, ${ }^{108}$ P. Antonioli, ${ }^{105}$ L. Aphecetche, ${ }^{113}$ H. Appelshäuser, ${ }^{53}$ S. Arcelli, ${ }^{28}$ N. Armesto, ${ }^{17}$ R. Arnaldi, ${ }^{111}$ I. C. Arsene, ${ }^{22}$ M. Arslandok, ${ }^{53}$ 
B. Audurier, ${ }^{113}$ A. Augustinus, ${ }^{36}$ R. Averbeck, ${ }^{97}$ M. D. Azmi, ${ }^{19}$ M. Bach, ${ }^{43}$ A. Badalà, ${ }^{107}$ Y. W. Baek, ${ }^{44}$ S. Bagnasco, ${ }^{111}$ R. Bailhache, ${ }^{53}$ R. Bala, ${ }^{90}$ A. Baldisseri, ${ }^{15}$ F. Baltasar Dos Santos Pedrosa, ${ }^{36}$ R. C. Baral, ${ }^{61}$ A. M. Barbano, ${ }^{11}$ R. Barbera, ${ }^{29}$ F. Barile, ${ }^{33}$ G. G. Barnaföldi, ${ }^{136}$ L. S. Barnby, ${ }^{102}$ V. Barret, ${ }^{70}$ P. Bartalini, ${ }^{7}$ K. Barth, ${ }^{36}$ J. Bartke, ${ }^{17}$ E. Bartsch, ${ }^{53}$ M. Basile, ${ }^{28}$ N. Bastid, ${ }^{70}$ S. Basu, ${ }^{132}$ B. Bathen, ${ }^{54}$ G. Batigne, ${ }^{113}$ A. Batista Camejo, ${ }^{70}$ B. Batyunya, ${ }^{66}$ P. C. Batzing, ${ }^{22}$ I. G. Bearden, ${ }^{80}$ H. Beck, ${ }^{53}$ C. Bedda, ${ }^{111}$ N. K. Behera, ${ }^{48,49}$ I. Belikov, ${ }^{55}$ F. Bellini, ${ }^{28}$ H. Bello Martinez, ${ }^{2}$ R. Bellwied, ${ }^{122}$ R. Belmont, ${ }^{135}$ E. Belmont-Moreno, ${ }^{64}$ V. Belyaev,${ }^{76}$ G. Bencedi, ${ }^{136}$ S. Beole,${ }^{27}$ I. Berceanu, ${ }^{78}$ A. Bercuci, ${ }^{78}$ Y. Berdnikov, ${ }^{85}$ D. Berenyi, ${ }^{136}$

R. A. Bertens, ${ }^{57}$ D. Berzano, ${ }^{36,27}$ L. Betev,${ }^{36}$ A. Bhasin, ${ }^{90}$ I. R. Bhat, ${ }^{90}$ A. K. Bhati, ${ }^{87}$ B. Bhattacharjee, ${ }^{45}$ J. Bhom, ${ }^{128}$

L. Bianchi, ${ }^{122}$ N. Bianchi, ${ }^{72}$ C. Bianchin, ${ }^{135,57}$ J. Bielčík, ${ }^{40}$ J. Bielčíková, ${ }^{83}$ A. Bilandzic, ${ }^{80}$ R. Biswas, ${ }^{4}$ S. Biswas, ${ }^{79}$ S. Bjelogrlic, ${ }^{57}$ J. T. Blair, ${ }^{118}$ F. Blanco, ${ }^{10}$ D. Blau, ${ }^{100}$ C. Blume, ${ }^{53}$ F. Bock,,${ }^{93,74}$ A. Bogdanov, ${ }^{76}$ H. Bøggild, ${ }^{80}$ L. Boldizsár, ${ }^{136}$ M. Bombara, ${ }^{41}$ J. Book, ${ }^{53}$ H. Borel, ${ }^{15}$ A. Borissov, ${ }^{96}$ M. Borri, ${ }^{82}$ F. Bossú, ${ }^{65}$ E. Botta, ${ }^{27}$ S. Böttger, ${ }^{52}$ P. Braun-Munzinger, ${ }^{97}$

M. Bregant, ${ }^{120}$ T. Breitner, ${ }^{52}$ T. A. Broker, ${ }^{53}$ T. A. Browning, ${ }^{95}$ M. Broz, ${ }^{40}$ E. J. Brucken, ${ }^{46}$ E. Bruna, ${ }^{111}$ G. E. Bruno, ${ }^{33}$

D. Budnikov, ${ }^{99}$ H. Buesching, ${ }^{53}$ S. Bufalino, ${ }^{111}$ P. Buncic, ${ }^{36}$ O. Busch, ${ }^{93,128}$ Z. Buthelezi, ${ }^{65}$ J. B. Butt, ${ }^{16}$ J. T. Buxton, ${ }^{20}$

D. Caffarri, ${ }^{36}$ X. Cai, ${ }^{7}$ H. Caines, ${ }^{137}$ L. Calero Diaz,${ }^{72}$ A. Caliva, ${ }^{57}$ E. Calvo Villar, ${ }^{103}$ P. Camerini, ${ }^{26}$ F. Carena, ${ }^{36}$ W. Carena, ${ }^{36}$

F. Carnesecchi, ${ }^{28}$ J. Castillo Castellanos, ${ }^{15}$ A. J. Castro, ${ }^{125}$ E. A. R. Casula, ${ }^{25}$ C. Cavicchioli, ${ }^{36}$ C. Ceballos Sanchez, ${ }^{9}$

J. Cepila, ${ }^{40}$ P. Cerello, ${ }^{111}$ J. Cerkala, ${ }^{115}$ B. Chang, ${ }^{123}$ S. Chapeland, ${ }^{36}$ M. Chartier, ${ }^{124}$ J. L. Charvet, ${ }^{15}$ S. Chattopadhyay, ${ }^{132}$

S. Chattopadhyay, ${ }^{101}$ V. Chelnokov,${ }^{3}$ M. Cherney, ${ }^{86}$ C. Cheshkov, ${ }^{130}$ B. Cheynis, ${ }^{130}$ V. Chibante Barroso, ${ }^{36}$ D. D. Chinellato, ${ }^{121}$

P. Chochula ${ }^{36}$ K. Choi, ${ }^{96}$ M. Chojnacki, ${ }^{80}$ S. Choudhury, ${ }^{132}$ P. Christakoglou, ${ }^{81}$ C. H. Christensen, ${ }^{80}$ P. Christiansen, ${ }^{34}$

T. Chujo, ${ }^{128}$ S. U. Chung, ${ }^{96}$ Z. Chunhui, ${ }^{57}$ C. Cicalo, ${ }^{106}$ L. Cifarelli, ${ }^{12,28}$ F. Cindolo, ${ }^{105}$ J. Cleymans, ${ }^{89}$ F. Colamaria, ${ }^{33}$

D. Colella, ${ }^{36,33,59}$ A. Collu, ${ }^{25}$ M. Colocci, ${ }^{28}$ G. Conesa Balbastre, ${ }^{71}$ Z. Conesa del Valle, ${ }^{51}$ M. E. Connors, ${ }^{137}$

J. G. Contreras, ${ }^{11,40}$ T. M. Cormier, ${ }^{84}$ Y. Corrales Morales, ${ }^{27}$ I. Cortés Maldonado, ${ }^{2}$ P. Cortese, ${ }^{32}$ M. R. Cosentino, ${ }^{120}$

F. Costa, ${ }^{36}$ P. Crochet,${ }^{70}$ R. Cruz Albino, ${ }^{11}$ E. Cuautle, ${ }^{63}$ L. Cunqueiro, ${ }^{36}$ T. Dahms, ${ }^{92,37}$ A. Dainese, ${ }^{108}$ A. Danu, ${ }^{62}$ D. Das, ${ }^{101}$

I. Das, ${ }^{51,101}$ S. Das, ${ }^{4}$ A. Dash, ${ }^{121}$ S. Dash, ${ }^{48}$ S. De, ${ }^{120}$ A. De Caro, ${ }^{31,12}$ G. de Cataldo, ${ }^{104}$ J. de Cuveland, ${ }^{43}$ A. De Falco, ${ }^{25}$

D. De Gruttola, ${ }^{12,31}$ N. De Marco, ${ }^{111}$ S. De Pasquale, ${ }^{31}$ A. Deisting, ${ }^{97,93}$ A. Deloff, ${ }^{77}$ E. Dénes, ${ }^{136}$ G. D'Erasmo, ${ }^{33}$ D. Di Bari, ${ }^{33}$

A. Di Mauro, ${ }^{36}$ P. Di Nezza, ${ }^{72}$ M. A. Diaz Corchero, ${ }^{10}$ T. Dietel, ${ }^{89}$ P. Dillenseger, ${ }^{53}$ R. Divià, ${ }^{36} \varnothing$. Djuvsland, ${ }^{18}$ A. Dobrin, ${ }^{57,81}$

T. Dobrowolski, ${ }^{77, *}$ D. Domenicis Gimenez, ${ }^{120}$ B. Dönigus, ${ }^{53}$ O. Dordic, ${ }^{22}$ T. Drozhzhova, ${ }^{53}$ A. K. Dubey, ${ }^{132}$ A. Dubla, ${ }^{57}$

L. Ducroux, ${ }^{130}$ P. Dupieux, ${ }^{70}$ R. J. Ehlers, ${ }^{137}$ D. Elia, ${ }^{104}$ H. Engel, ${ }^{52}$ B. Erazmus, ${ }^{36,113}$ I. Erdemir, ${ }^{53}$ F. Erhardt, ${ }^{129}$

D. Eschweiler, ${ }^{43}$ B. Espagnon, ${ }^{51}$ M. Estienne, ${ }^{113}$ S. Esumi, ${ }^{128}$ J. Eum, ${ }^{96}$ D. Evans, ${ }^{102}$ S. Evdokimov, ${ }^{112}$ G. Eyyubova, ${ }^{40}$

L. Fabbietti, ${ }^{37,92}$ D. Fabris, ${ }^{108}$ J. Faivre, ${ }^{71}$ A. Fantoni, ${ }^{72}$ M. Fasel, ${ }^{74}$ L. Feldkamp,${ }^{54}$ D. Felea, ${ }^{62}$ A. Feliciello, ${ }^{111}$ G. Feofilov, ${ }^{131}$

J. Ferencei, ${ }^{83}$ A. Fernández Téllez, ${ }^{2}$ E. G. Ferreiro, ${ }^{17}$ A. Ferretti, ${ }^{27}$ A. Festanti, ${ }^{30}$ V. J. G. Feuillard, ${ }^{70,15}$ J. Figiel, ${ }^{117}$

M. A. S. Figueredo, ${ }^{124,120}$ S. Filchagin, ${ }^{99}$ D. Finogeev, ${ }^{56}$ E. M. Fiore, ${ }^{33}$ M. G. Fleck, ${ }^{93}$ M. Floris, ${ }^{36}$ S. Foertsch, ${ }^{65}$ P. Foka, ${ }^{97}$ S. Fokin, ${ }^{100}$ E. Fragiacomo, ${ }^{10}$ A. Francescon, ${ }^{30,36}$ U. Frankenfeld, ${ }^{97}$ U. Fuchs, ${ }^{36}$ C. Furget,${ }^{71}$ A. Furs, ${ }^{56}$ M. Fusco Girard, ${ }^{31}$ J. J. Gaardhøje, ${ }^{80}$ M. Gagliardi, ${ }^{27}$ A. M. Gago, ${ }^{103}$ M. Gallio, ${ }^{27}$ D. R. Gangadharan, ${ }^{74}$ P. Ganoti, ${ }^{88}$ C. Gao, ${ }^{7}$ C. Garabatos, ${ }^{97}$

E. Garcia-Solis, ${ }^{13}$ C. Gargiulo, ${ }^{36}$ P. Gasik, ${ }^{92,37}$ M. Germain, ${ }^{113}$ A. Gheata, ${ }^{36}$ M. Gheata, ${ }^{62,36}$ P. Ghosh, ${ }^{132}$ S. K. Ghosh, P. Gianotti, ${ }^{72}$ P. Giubellino, ${ }^{36,111}$ P. Giubilato, ${ }^{30}$ E. Gladysz-Dziadus, ${ }^{117}$ P. Glässel,,${ }^{93}$ D. M. Goméz Coral, ${ }^{64}$

A. Gomez Ramirez, ${ }^{52}$ P. González-Zamora, ${ }^{10}$ S. Gorbunov, ${ }^{43}$ L. Görlich, ${ }^{117}$ S. Gotovac, ${ }^{116}$ V. Grabski, ${ }^{64}$

L. K. Graczykowski, ${ }^{134}$ K. L. Graham, ${ }^{12}$ A. Grelli, ${ }^{57}$ A. Grigoras, ${ }^{36}$ C. Grigoras, ${ }^{36}$ V. Grigoriev, ${ }^{76}$ A. Grigoryan, ${ }^{1}$

S. Grigoryan, ${ }^{66}$ B. Grinyov, ${ }^{3}$ N. Grion, ${ }^{110}$ J. F. Grosse-Oetringhaus, ${ }^{36}$ J.-Y. Grossiord, ${ }^{130}$ R. Grosso, ${ }^{36}$ F. Guber, ${ }^{56}$

R. Guernane, ${ }^{71}$ B. Guerzoni, ${ }^{28}$ K. Gulbrandsen, ${ }^{80}$ H. Gulkanyan, ${ }^{1}$ T. Gunji, ${ }^{127}$ A. Gupta, ${ }^{90}$ R. Gupta, ${ }^{90}$ R. Haake, ${ }^{54}$

Ø. Haaland, ${ }^{18}$ C. Hadjidakis, ${ }^{51}$ M. Haiduc, ${ }^{62}$ H. Hamagaki, ${ }^{127}$ G. Hamar, ${ }^{136}$ A. Hansen, ${ }^{80}$ J. W. Harris, ${ }^{137}$ H. Hartmann, ${ }^{43}$

A. Harton, ${ }^{13}$ D. Hatzifotiadou, ${ }^{105}$ S. Hayashi, ${ }^{127}$ S. T. Heckel, ${ }^{53}$ M. Heide,${ }^{54}$ H. Helstrup, ${ }^{38}$ A. Herghelegiu, ${ }^{78}$

G. Herrera Corral, ${ }^{11}$ B. A. Hess, ${ }^{35}$ K. F. Hetland, ${ }^{38}$ T. E. Hilden, ${ }^{46}$ H. Hillemanns, ${ }^{36}$ B. Hippolyte, ${ }^{55}$ R. Hosokawa, ${ }^{128}$

P. Hristov, ${ }^{36}$ M. Huang, ${ }^{18}$ T. J. Humanic, ${ }^{20}$ N. Hussain, ${ }^{45}$ T. Hussain, ${ }^{19}$ D. Hutter, ${ }^{43}$ D. S. Hwang, ${ }^{21}$ R. Ilkaev, ${ }^{99}$ I. Ilkiv, ${ }^{77}$

M. Inaba, ${ }^{128}$ M. Ippolitov, ${ }^{76,100}$ M. Irfan, ${ }^{19}$ M. Ivanov, ${ }^{97}$ V. Ivanov, ${ }^{85}$ V. Izucheev, ${ }^{112}$ P. M. Jacobs ${ }^{74} \mathrm{~S}$. Jadlovska, ${ }^{115}$

C. Jahnke, ${ }^{120}$ H. J. Jang, ${ }^{68}$ M. A. Janik, ${ }^{134}$ P. H. S. Y. Jayarathna, ${ }^{122}$ C. Jena, ${ }^{30}$ S. Jena, ${ }^{122}$ R. T. Jimenez Bustamante, ${ }^{97}$

P. G. Jones, ${ }^{102}$ H. Jung, ${ }^{44}$ A. Jusko, ${ }^{102}$ P. Kalinak, ${ }^{59}$ A. Kalweit, ${ }^{36}$ J. Kamin, ${ }^{53}$ J. H. Kang, ${ }^{138}$ V. Kaplin, ${ }^{76}$ S. Kar, ${ }^{132}$

A. Karasu Uysal, ${ }^{6}$ O. Karavichev, ${ }^{56}$ T. Karavicheva, ${ }^{56}$ L. Karayan, ${ }^{93,97}$ E. Karpechev, ${ }^{56}$ U. Kebschull, ${ }^{52}$ R. Keidel, ${ }^{139}$

D. L. D. Keijdener, ${ }^{57}$ M. Keil,${ }^{36}$ K. H. Khan, ${ }^{16}$ M. M. Khan, ${ }^{19}$ P. Khan, ${ }^{101}$ S. A. Khan, ${ }^{132}$ A. Khanzadeev, ${ }^{85}$ Y. Kharlov, ${ }^{112}$

B. Kileng, ${ }^{38}$ B. Kim, ${ }^{138}$ D. W. Kim,${ }^{68,44}$ D. J. Kim, ${ }^{123}$ H. Kim, ${ }^{138}$ J. S. Kim, ${ }^{44}$ M. Kim, ${ }^{44}$ M. Kim, ${ }^{138}$ S. Kim, ${ }^{21}$ T. Kim, ${ }^{138}$

S. Kirsch, ${ }^{43}$ I. Kisel, ${ }^{43}$ S. Kiselev, ${ }^{58}$ A. Kisiel, ${ }^{134}$ G. Kiss, ${ }^{136}$ J. L. Klay, ${ }^{6}$ C. Klein, ${ }^{53}$ J. Klein, ${ }^{36,93}$ C. Klein-Bösing, ${ }^{54}$

A. Kluge ${ }^{36}$ M. L. Knichel, ${ }^{93}$ A. G. Knospe, ${ }^{118}$ T. Kobayashi, ${ }^{128}$ C. Kobdaj, ${ }^{114}$ M. Kofarago, ${ }^{36}$ T. Kollegger, ${ }^{43,97}$

A. Kolojvari, ${ }^{131}$ V. Kondratiev, ${ }^{131}$ N. Kondratyeva, ${ }^{76}$ E. Kondratyuk, ${ }^{112}$ A. Konevskikh, ${ }^{56}$ M. Kopcik, ${ }^{115}$ M. Kour, ${ }^{90}$

C. Kouzinopoulos, ${ }^{36}$ O. Kovalenko, ${ }^{77}$ V. Kovalenko, ${ }^{131}$ M. Kowalski, ${ }^{117}$ G. Koyithatta Meethaleveedu, ${ }^{48}$ J. Kral, ${ }^{123}$ I. Králik,${ }^{59}$ A. Kravčáková, ${ }^{41}$ M. Krelina, ${ }^{40}$ M. Kretz, ${ }^{43}$ M. Krivda, ${ }^{102,59}$ F. Krizek, ${ }^{83}$ E. Kryshen, ${ }^{36}$ M. Krzewicki, ${ }^{43}$ A. M. Kubera, ${ }^{20}$

V. Kučera, ${ }^{83}$ T. Kugathasan, ${ }^{36}$ C. Kuhn, ${ }^{55}$ P. G. Kuijer, ${ }^{81}$ I. Kulakov, ${ }^{43}$ A. Kumar, ${ }^{90}$ J. Kumar, ${ }^{48}$ L. Kumar, ${ }^{79,87}$ P. Kurashvili, ${ }^{77}$

A. Kurepin, ${ }^{56}$ A. B. Kurepin, ${ }^{56}$ A. Kuryakin, ${ }^{99}$ S. Kushpil, ${ }^{83}$ M. J. Kweon, ${ }^{50}$ Y. Kwon, ${ }^{138}$ S. L. La Pointe, ${ }^{111}$ P. La Rocca, ${ }^{29}$

C. Lagana Fernandes, ${ }^{120}$ I. Lakomov, ${ }^{36}$ R. Langoy, ${ }^{42}$ C. Lara, ${ }^{52}$ A. Lardeux, ${ }^{15}$ A. Lattuca, ${ }^{27}$ E. Laudi, ${ }^{36}$ R. Lea, ${ }^{26}$ L. Leardini, ${ }^{93}$ G. R. Lee, ${ }^{102}$ S. Lee, ${ }^{138}$ I. Legrand, ${ }^{36}$ F. Lehas, ${ }^{81}$ R. C. Lemmon, ${ }^{82}$ V. Lenti, ${ }^{104}$ E. Leogrande, ${ }^{57}$ I. León Monzón, ${ }^{119}$ M. Leoncino, ${ }^{27}$ P. Lévai, ${ }^{136} \mathrm{~S} . \mathrm{Li},{ }^{7,70} \mathrm{X} . \mathrm{Li},{ }^{14} \mathrm{~J}$. Lien, ${ }^{42}$ R. Lietava, ${ }^{102} \mathrm{~S}$. Lindal, ${ }^{22} \mathrm{~V}$. Lindenstruth, ${ }^{43} \mathrm{C}$. Lippmann, ${ }^{97}$ 
M. A. Lisa, ${ }^{20}$ H. M. Ljunggren, ${ }^{34}$ D. F. Lodato, ${ }^{57}$ P. I. Loenne, ${ }^{18}$ V. Loginov,${ }^{76}$ C. Loizides,${ }^{74}$ X. Lopez,${ }^{70}$ E. López Torres, ${ }^{9}$ A. Lowe, ${ }^{136}$ P. Luettig, ${ }^{53}$ M. Lunardon, ${ }^{30}$ G. Luparello, ${ }^{26}$ P. H. F. N. D. Luz, ${ }^{120}$ A. Maevskaya ${ }^{56}$ M. Mager, ${ }^{36}$ S. Mahajan, ${ }^{90}$ S. M. Mahmood, ${ }^{22}$ A. Maire, ${ }^{55}$ R. D. Majka, ${ }^{137}$ M. Malaev,${ }^{85}$ I. Maldonado Cervantes, ${ }^{63}$ L. Malinina $,{ }^{66},{ }^{\dagger}$ D. Mal'Kevich,${ }^{58}$ P. Malzacher ${ }^{97}$ A. Mamonov, ${ }^{99}$ V. Manko, ${ }^{100}$ F. Manso,${ }^{70}$ V. Manzari, ${ }^{36,104}$ M. Marchisone,${ }^{27}$ J. Mareš, ${ }^{60}$ G. V. Margagliotti,${ }^{26}$ A. Margotti, ${ }^{105}$ J. Margutti, ${ }^{57}$ A. Marín,,${ }^{97}$ C. Markert, ${ }^{118}$ M. Marquard, ${ }^{53}$ N. A. Martin, ${ }^{97}$ J. Martin Blanco,,${ }^{113}$ P. Martinengo, ${ }^{36}$ M. I. Martínez, ${ }^{2}$ G. Martínez García, ${ }^{113}$ M. Martinez Pedreira, ${ }^{36}$ Y. Martynov,${ }^{3}$ A. Mas, ${ }^{120}$ S. Masciocchi, ${ }^{97}$ M. Masera, ${ }^{27}$ A. Masoni, ${ }^{106}$ L. Massacrier, ${ }^{113}$ A. Mastroserio, ${ }^{33}$ H. Masui, ${ }^{128}$ A. Matyja, ${ }^{117}$ C. Mayer, ${ }^{117}$ J. Mazer,${ }^{125}$ M. A. Mazzoni, ${ }^{109}$ D. Mcdonald, ${ }^{122}$ F. Meddi, ${ }^{24}$ Y. Melikyan, ${ }^{76}$ A. Menchaca-Rocha,${ }^{64}$ E. Meninno,${ }^{31}$ J. Mercado Pérez, ${ }^{93}$ M. Meres,${ }^{39}$ Y. Miake, ${ }^{128}$ M. M. Mieskolainen, ${ }^{46}$ K. Mikhaylov, ${ }^{58,66}$ L. Milano, ${ }^{36}$ J. Milosevic, ${ }^{22,}, 133$ L. M. Minervini, ${ }^{104,23}$ A. Mischke, ${ }^{57}$ A. N. Mishra, ${ }^{49}$ D. Miśkowiec, ${ }^{97}$ J. Mitra, ${ }^{132}$ C. M. Mitu, ${ }^{62}$ N. Mohammadi, ${ }^{57}$ B. Mohanty,${ }^{132,79}$ L. Molnar,${ }^{55}$

L. Montaño Zetina, ${ }^{11}$ E. Montes,${ }^{10}$ M. Morando ${ }^{30}$ D. A. Moreira De Godoy,${ }^{113,54}$ S. Moretto, ${ }^{30}$ A. Morreale,,${ }^{113}$ A. Morsch,${ }^{36}$ V. Muccifora, ${ }^{72}$ E. Mudnic, ${ }^{116}$ D. Mühlheim, ${ }^{54}$ S. Muhuri, ${ }^{132}$ M. Mukherjee,,${ }^{132}$ J. D. Mulligan,,${ }^{137}$ M. G. Munhoz, ${ }^{120}$ S. Murray ${ }^{65}$ L. Musa, ${ }^{36}$ J. Musinsky, ${ }^{59}$ B. K. Nandi, ${ }^{48}$ R. Nania, ${ }^{105}$ E. Nappi, ${ }^{104}$ M. U. Naru ${ }^{16}$ C. Nattrass, ${ }^{125}$ K. Nayak,${ }^{79}$ T. K. Nayak, ${ }^{132}$ S. Nazarenko, ${ }^{99}$ A. Nedosekin, ${ }^{58}$ L. Nellen, ${ }^{63}$ F. Ng, ${ }^{122}$ M. Nicassio, ${ }^{97}$ M. Niculescu, ${ }^{62,36}$ J. Niedziela, ${ }^{36}$ B. S. Nielsen ${ }^{80}$ S. Nikolaev, ${ }^{100}$ S. Nikulin, ${ }^{100}$ V. Nikulin, ${ }^{85}$ F. Noferini, ${ }^{105,12}$ P. Nomokonov, ${ }^{66}$ G. Nooren, ${ }^{57}$ J. C. C. Noris, ${ }^{2}$ J. Norman, ${ }^{124}$ A. Nyanin, ${ }^{100}$ J. Nystrand, ${ }^{18}$ H. Oeschler, ${ }^{93}$ S. Oh, ${ }^{137}$ S. K. Oh, ${ }^{67}$ A. Ohlson, ${ }^{36}$ A. Okatan, ${ }^{69}$ T. Okubo, ${ }^{47}$ L. Olah, ${ }^{136}$ J. Oleniacz, ${ }^{134}$ A. C. Oliveira Da Silva ${ }^{120}$ M. H. Oliver, ${ }^{137}$ J. Onderwaater, ${ }^{97}$ C. Oppedisano, ${ }^{111}$ R. Orava, ${ }^{46}$ A. Ortiz Velasquez, ${ }^{63}$ A. Oskarsson, ${ }^{34}$ J. Otwinowski, ${ }^{117}$ K. Oyama, ${ }^{93}$ M. Ozdemir, ${ }^{53}$ Y. Pachmayer,${ }^{93}$ P. Pagano, ${ }^{31}$ G. Paić,, 33 C. Pajares,${ }^{17}$ S. K. Pal, ${ }^{132}$ J. Pan, ${ }^{135}$ A. K. Pandey ${ }^{48}$ D. Pant, ${ }^{48}$ P. Papcun, ${ }^{115}$ V. Papikyan, ${ }^{1}$ G. S. Pappalardo, ${ }^{107}$ P. Pareek, ${ }^{49}$ W. J. Park, ${ }^{97}$ S. Parmar, ${ }^{87}$ A. Passfeld,,${ }^{54}$ V. Paticchio, ${ }^{104}$ R. N. Patra, ${ }^{132}$ B. Paul, ${ }^{101}$ T. Peitzmann,,${ }^{57}$ H. Pereira Da Costa, ${ }^{15}$

E. Pereira De Oliveira Filho, ${ }^{120}$ D. Peresunko, ${ }^{100,76}$ C. E. Pérez Lara, ${ }^{81}$ E. Perez Lezama, ${ }^{53}$ V. Peskov, ${ }^{53}$ Y. Pestov, ${ }^{5}$ V. Petráček, ${ }^{40}$ V. Petrov, ${ }^{112}$ M. Petrovici, ${ }^{78}$ C. Petta, ${ }^{29}$ S. Piano, ${ }^{110}$ M. Pikna, ${ }^{39}$ P. Pillot, ${ }^{13}$ O. Pinazza, ${ }^{105}, 36$ L. Pinsky, ${ }^{122}$ D. B. Piyarathna, ${ }^{122}$ M. Płoskoń, ${ }^{74}$ M. Planinic, ${ }^{129}$ J. Pluta, ${ }^{134}$ S. Pochybova ${ }^{136}$ P. L. M. Podesta-Lerma,,${ }^{119}$ M. G. Poghosyan ${ }^{86,84}$ B. Polichtchouk, ${ }^{112}$ N. Poljak, ${ }^{129}$ W. Poonsawat, ${ }^{114}$ A. Pop, ${ }^{78}$ S. Porteboeuf-Houssais ${ }^{70}$ J. Porter ${ }^{74}$ J. Pospisil,${ }^{83}$ S. K. Prasad, ${ }^{4}$ R. Preghenella ${ }^{36,105}$ F. Prino, ${ }^{111}$ C. A. Pruneau, ${ }^{135}$ I. Pshenichnov ${ }^{56}$ M. Puccio, ${ }^{111}$ G. Puddu, ${ }^{25}$

P. Pujahari, ${ }^{135}$ V. Punin, ${ }^{99}$ J. Putschke, ${ }^{135}$ H. Qvigstad, ${ }^{22}$ A. Rachevski, ${ }^{110}$ S. Raha,${ }^{4}$ S. Rajput, ${ }^{90}$ J. Rak,,${ }^{123}$

A. Rakotozafindrabe, ${ }^{15}$ L. Ramello, ${ }^{32}$ R. Raniwala ${ }^{91}$ S. Raniwala, ${ }^{91}$ S. S. Räsänen, ${ }^{46}$ B. T. Rascanu, ${ }^{53}$ D. Rathee, ${ }^{87}$

K. F. Read, ${ }^{125}$ J. S. Real, ${ }^{71}$ K. Redlich, ${ }^{77}$ R. J. Reed, ${ }^{135}$ A. Rehman, ${ }^{18}$ P. Reichelt,${ }^{53}$ F. Reidt,${ }^{93,36}$ X. Ren, ${ }^{7}$ R. Renfordt, ${ }^{53}$ A. R. Reolon, ${ }^{72}$ A. Reshetin, ${ }^{56}$ F. Rettig, ${ }^{43}$ J.-P. Revol, ${ }^{12}$ K. Reygers, ${ }^{93}$ V. Riabov, ${ }^{85}$ R. A. Ricci, ${ }^{73}$ T. Richert, ${ }^{34}$ M. Richter, ${ }^{22}$ P. Riedler, ${ }^{36}$ W. Riegler, ${ }^{36}$ F. Riggi, ${ }^{29}$ C. Ristea, ${ }^{62}$ A. Rivetti, ${ }^{111}$ E. Rocco,${ }^{57}$ M. Rodríguez Cahuantzi, ${ }^{2}$ A. Rodriguez Manso, ${ }^{81}$ K. Røed, ${ }^{22}$ E. Rogochaya, ${ }^{66}$ D. Rohr ${ }^{43}$ D. Röhrich, ${ }^{18}$ R. Romita, ${ }^{124}$ F. Ronchetti, ${ }^{72}$ L. Ronflette, ${ }^{113}$ P. Rosnet, ${ }^{70}$ A. Rossi, ${ }^{30,36}$ F. Roukoutakis,${ }^{88}$ A. Roy, ${ }^{49}$ C. Roy ${ }^{55}$ P. Roy ${ }^{101}$ A. J. Rubio Montero, ${ }^{10}$ R. Rui ${ }^{26}$ R. Russo, ${ }^{27}$ E. Ryabinkin,,${ }^{100}$ Y. Ryabov ${ }^{85}$ A. Rybicki, ${ }_{117}$ S. Sadovsky, ${ }^{112}$ K. Šafařík ${ }^{36}$ B. Sahlmuller, ${ }^{53}$ P. Sahoo, ${ }^{49}$ R. Sahoo ${ }^{49}$ S. Sahoo, ${ }^{61}$ P. K. Sahu, ${ }^{61}$ J. Saini, ${ }^{132}$ S. Sakai, ${ }^{72}$ M. A. Saleh, ${ }^{135}$ C. A. Salgado, ${ }^{17}$ J. Salzwedel, ${ }^{20}$ S. Sambyal, ${ }^{90}$ V. Samsonov, ${ }^{85}$ X. Sanchez Castro, ${ }^{55}$ L. Šándor, ${ }^{59}$ A. Sandoval, ${ }^{64}$ M. Sano, ${ }^{128}$ D. Sarkar, ${ }^{132}$ E. Scapparone,,${ }^{105}$ F. Scarlassara, ${ }^{30}$ R. P. Scharenberg, ${ }^{95}$ C. Schiaua ${ }^{78}$ R. Schicker,${ }^{93}$ C. Schmidt, ${ }^{97}$ H. R. Schmidt, ${ }^{35}$ S. Schuchmann, ${ }^{53}$ J. Schukraft, ${ }^{36}$ M. Schulc,,${ }^{40}$ T. Schuster, ${ }^{137}$ Y. Schutz, ${ }^{13,36}$ K. Schwarz, ${ }^{97}$ K. Schweda, ${ }^{97}$ G. Scioli, ${ }^{28}$ E. Scomparin,, 11 R. Scott, ${ }^{125}$ J. E. Seger, ${ }^{86}$ Y. Sekiguchi, ${ }^{127}$ D. Sekihata, ${ }^{47}$ I. Selyuzhenkov, ${ }^{97}$ K. Senosi, ${ }^{65}$ J. Seo, ${ }^{96,67}$ E. Serradilla ${ }^{64,10}$ A. Sevcenco, ${ }^{62}$ A. Shabanov, ${ }^{56}$ A. Shabetai, ${ }^{113}$ O. Shadura, ${ }^{3}$ R. Shahoyan, ${ }^{36}$ A. Shangaraev, ${ }^{112}$ A. Sharma, ${ }^{90}$ M. Sharma,${ }^{90}$ M. Sharma, ${ }^{90}$ N. Sharma, ${ }^{125,61}$ K. Shigaki, ${ }^{47}$ K. Shtejer, ${ }^{9,27}$ Y. Sibiriak, ${ }^{100}$ S. Siddhanta, ${ }^{106}$ K. M. Sielewicz, ${ }^{36}$ T. Siemiarczuk, ${ }^{77}$ D. Silvermyr, ${ }^{84,34}$ C. Silvestre, ${ }^{71}$ G. Simatovic, ${ }^{129}$ G. Simonetti, ${ }^{36}$ R. Singaraju, ${ }^{132}$ R. Singh,${ }^{79}$ S. Singha, ${ }^{132,79}$ V. Singhal, ${ }^{132}$ B. C. Sinha, ${ }^{132}$ T. Sinha, ${ }^{101}$ B. Sitar, ${ }^{39}$ M. Sitta, ${ }^{32}$ T. B. Skaali, ${ }^{22}$ M. Slupecki, ${ }^{123}$ N. Smirnov, ${ }^{137}$ R. J. M. Snellings, ${ }^{57}$ T. W. Snellman, ${ }^{123}$ C. Søgaard, ${ }^{34}$ R. Soltz, ${ }^{75}$ J. Song, ${ }^{96}$ M. Song, ${ }^{138}$ Z. Song, ${ }^{7}$ F. Soramel, ${ }^{30}$ S. Sorensen, ${ }^{125}$ M. Spacek, ${ }^{40}$ E. Spiriti ${ }^{72}$ I. Sputowska, ${ }^{117}$ M. Spyropoulou-Stassinaki, ${ }^{88}$ B. K. Srivastava, ${ }^{95}$ J. Stachel, ${ }^{93}$ I. Stan, ${ }^{62}$ G. Stefanek, ${ }^{77}$ M. Steinpreis, ${ }^{20}$ E. Stenlund, ${ }^{34}$ G. Steyn,,${ }^{65}$ J. H. Stiller ${ }^{93}$ D. Stocco,,${ }^{113}$ P. Strmen, ${ }^{39}$ A. A. P. Suaide, ${ }^{120}$ T. Sugitate, ${ }^{47}$ C. Suire, ${ }^{51}$ M. Suleymanov, ${ }^{16}$ R. Sultanov, ${ }^{58}$ M. Sumbera, ${ }^{83}$ T. J. M. Symons,${ }^{74}$ A. Szabo, ${ }^{39}$ A. Szanto de Toledo, ${ }^{120, *}$ I. Szarka, ${ }^{39}$ A. Szczepankiewicz, ${ }^{36}$ M. Szymanski, ${ }^{134}$ J. Takahashi, ${ }^{121}$ G. J. Tambave, ${ }^{18}$ N. Tanaka, ${ }^{128}$ M. A. Tangaro, ${ }^{33}$ J. D. Tapia Takaki, ${ }^{51,+}$ A. Tarantola Peloni, ${ }^{53}$ M. Tarhini, ${ }^{51}$ M. Tariq, ${ }^{19}$ M. G. Tarzila, ${ }^{78}$ A. Tauro, ${ }^{36}$ G. Tejeda Muñoz, ${ }^{2}$ A. Telesca, ${ }^{36}$ K. Terasaki, ${ }^{127}$ C. Terrevoli,,${ }^{30,25}$ B. Teyssier, ${ }^{130}$ J. Thäder,${ }^{74,97}$ D. Thomas, ${ }^{118}$ R. Tieulent, ${ }^{130}$ A. R. Timmins, ${ }^{122}$ A. Toia, ${ }^{53}$ S. Trogolo, ${ }^{111}$ V. Trubnikov, ${ }^{3}$ W. H. Trzaska, ${ }^{123}$ T. Tsuji, ${ }^{127}$ A. Tumkin, ${ }^{99}$ R. Turrisi, ${ }^{108}$ T. S. Tveter, ${ }^{22}$ K. Ullaland, ${ }^{18}$ A. Uras, ${ }^{130}$ G. L. Usai, ${ }^{25}$ A. Utrobicic, ${ }^{129}$ M. Vajzer, ${ }^{83}$ M. Vala, ${ }^{59}$ L. Valencia Palomo, ${ }^{70}$ S. Vallero, ${ }^{27}$ J. Van Der Maarel,${ }^{57}$ J. W. Van Hoorne, ${ }^{36}$ M. van Leeuwen, ${ }^{57}$ T. Vanat, ${ }^{83}$ P. Vande Vyvre, ${ }^{36}$ D. Varga, ${ }^{136}$ A. Vargas, ${ }^{2}$ M. Vargyas, ${ }^{123}$ R. Varma,${ }^{48}$ M. Vasileiou, ${ }^{88}$ A. Vasiliev, ${ }^{100}$ A. Vauthier,${ }^{71}$ V. Vechernin,${ }^{131}$

A. M. Veen, ${ }^{57}$ M. Veldhoen, ${ }^{57}$ A. Velure, ${ }^{18}$ M. Venaruzzo, ${ }^{73}$ E. Vercellin, ${ }^{27}$ S. Vergara Limón, ${ }^{2}$ R. Vernet,${ }^{8}$ M. Verweij, ${ }^{135,36}$ L. Vickovic, ${ }^{116}$ G. Viesti, ${ }^{30,}$ J. Viinikainen, ${ }^{123}$ Z. Vilakazi, ${ }^{126}$ O. Villalobos Baillie, ${ }^{102}$ A. Vinogradov, ${ }^{100}$ L. Vinogradov, ${ }^{131}$ Y. Vinogradov, ${ }^{99, *}$ T. Virgili, ${ }^{31}$ V. Vislavicius,${ }^{34}$ Y. P. Viyogi, ${ }^{132}$ A. Vodopyanov, ${ }^{66}$ M. A. Völkl, ${ }^{93}$ K. Voloshin,${ }^{58}$ S. A. Voloshin, ${ }^{135}$ G. Volpe, ${ }^{136,36}$ B. von Haller, ${ }^{36}$ I. Vorobyev, ${ }^{37,92}$ D. Vranic, ${ }^{36,97}$ J. Vrláková, ${ }^{41}$ B. Vulpescu, ${ }^{70}$ A. Vyushin, ${ }^{99}$ B. Wagner, ${ }^{18}$ J. Wagner,${ }^{97}$ H. Wang, ${ }^{57}$ M. Wang, ${ }^{7,113}$ Y. Wang,${ }^{93}$ D. Watanabe, ${ }^{128}$ Y. Watanabe, ${ }^{127}$ M. Weber, ${ }^{36}$ S. G. Weber, ${ }^{97}$ J. P. Wessels, ${ }^{54}$ U. Westerhoff, ${ }^{54}$ J. Wiechula, ${ }^{35}$ J. Wikne, ${ }^{22}$ M. Wilde, ${ }^{54}$ G. Wilk, ${ }^{77}$ J. Wilkinson,,${ }^{93}$ 
M. C. S. Williams, ${ }^{105}$ B. Windelband, ${ }^{93}$ M. Winn, ${ }^{93}$ C. G. Yaldo, ${ }^{135}$ H. Yang, ${ }^{57}$ P. Yang, ${ }^{7}$ S. Yano, ${ }^{47}$ Z. Yin, ${ }^{7}$ H. Yokoyama, ${ }^{128}$ I.-K. Yoo, ${ }^{96}$ V. Yurchenko, ${ }^{3}$ I. Yushmanov, ${ }^{100}$ A. Zaborowska, ${ }^{134}$ V. Zaccolo, ${ }^{80}$ A. Zaman, ${ }^{16}$ C. Zampolli, ${ }^{105}$ H. J. C. Zanoli, ${ }^{120}$ S. Zaporozhets, ${ }^{66}$ N. Zardoshti, ${ }^{102}$ A. Zarochentsev, ${ }^{131}$ P. Závada ${ }^{60}$ N. Zaviyalov, ${ }^{99}$ H. Zbroszczyk, ${ }^{134}$ I. S. Zgura, ${ }^{62}$

M. Zhalov, ${ }^{85}$ H. Zhang, ${ }^{18,7}$ X. Zhang, ${ }^{74}$ Y. Zhang, ${ }^{7}$ C. Zhao, ${ }^{22}$ N. Zhigareva, ${ }^{58}$ D. Zhou, ${ }^{7}$ Y. Zhou, ${ }^{80,57}$ Z. Zhou, ${ }^{18}$ H. Zhu, ${ }^{18,7}$ J. Zhu, ${ }^{113,7}$ X. Zhu, ${ }^{7}$ A. Zichichi, ${ }^{12,28}$ A. Zimmermann, ${ }^{93}$ M. B. Zimmermann, ${ }^{54,36}$ G. Zinovjev, ${ }^{3}$ and M. Zyzak ${ }^{43}$

(ALICE Collaboration)

\footnotetext{
${ }^{1}$ A.I. Alikhanyan National Science Laboratory (Yerevan Physics Institute) Foundation, Yerevan, Armenia

${ }^{2}$ Benemérita Universidad Autónoma de Puebla, Puebla, Mexico

${ }^{3}$ Bogolyubov Institute for Theoretical Physics, Kiev, Ukraine

${ }^{4}$ Bose Institute, Department of Physics and Centre for Astroparticle Physics and Space Science (CAPSS), Kolkata, India

${ }^{5}$ Budker Institute for Nuclear Physics, Novosibirsk, Russia

${ }^{6}$ California Polytechnic State University, San Luis Obispo, California, USA

${ }^{7}$ Central China Normal University, Wuhan, China

${ }^{8}$ Centre de Calcul de l'IN2P3, Villeurbanne, France

${ }^{9}$ Centro de Aplicaciones Tecnológicas y Desarrollo Nuclear (CEADEN), Havana, Cuba

${ }^{10}$ Centro de Investigaciones Energéticas Medioambientales y Tecnológicas (CIEMAT), Madrid, Spain

${ }^{11}$ Centro de Investigación y de Estudios Avanzados (CINVESTAV), Mexico City and Mérida, Mexico

${ }^{12}$ Centro Fermi - Museo Storico della Fisica e Centro Studi e Ricerche "Enrico Fermi", Rome, Italy

${ }^{13}$ Chicago State University, Chicago, Illinois, USA

${ }^{14}$ China Institute of Atomic Energy, Beijing, China

${ }^{15}$ Commissariat à l'Energie Atomique, IRFU, Saclay, France

${ }^{16}$ COMSATS Institute of Information Technology (CIIT), Islamabad, Pakistan

${ }^{17}$ Departamento de Física de Partículas and IGFAE, Universidad de Santiago de Compostela, Santiago de Compostela, Spain

${ }^{18}$ Department of Physics and Technology, University of Bergen, Bergen, Norway

${ }^{19}$ Department of Physics, Aligarh Muslim University, Aligarh, India

${ }^{20}$ Department of Physics, Ohio State University, Columbus, Ohio, USA

${ }^{21}$ Department of Physics, Sejong University, Seoul, South Korea

${ }^{22}$ Department of Physics, University of Oslo, Oslo, Norway

${ }^{23}$ Dipartimento di Elettrotecnica ed Elettronica del Politecnico, Bari, Italy

${ }^{24}$ Dipartimento di Fisica dell'Università 'La Sapienza' and Sezione INFN Rome, Italy

${ }^{25}$ Dipartimento di Fisica dell'Università and Sezione INFN, Cagliari, Italy

${ }^{26}$ Dipartimento di Fisica dell'Università and Sezione INFN, Trieste, Italy

${ }^{27}$ Dipartimento di Fisica dell'Università and Sezione INFN, Turin, Italy

${ }^{28}$ Dipartimento di Fisica e Astronomia dell'Università and Sezione INFN, Bologna, Italy

${ }^{29}$ Dipartimento di Fisica e Astronomia dell'Università and Sezione INFN, Catania, Italy

${ }^{30}$ Dipartimento di Fisica e Astronomia dell'Università and Sezione INFN, Padova, Italy

${ }^{31}$ Dipartimento di Fisica 'E.R. Caianiello' dell'Università and Gruppo Collegato INFN, Salerno, Italy

${ }^{32}$ Dipartimento di Scienze e Innovazione Tecnologica dell'Università del Piemonte Orientale and Gruppo Collegato INFN, Alessandria, Italy

${ }^{33}$ Dipartimento Interateneo di Fisica 'M. Merlin' and Sezione INFN, Bari, Italy

${ }^{34}$ Division of Experimental High Energy Physics, University of Lund, Lund, Sweden

${ }^{35}$ Eberhard Karls Universität Tübingen, Tübingen, Germany

${ }^{36}$ European Organization for Nuclear Research (CERN), Geneva, Switzerland

${ }^{37}$ Excellence Cluster Universe, Technische Universität München, Munich, Germany

${ }^{38}$ Faculty of Engineering, Bergen University College, Bergen, Norway

${ }^{39}$ Faculty of Mathematics, Physics and Informatics, Comenius University, Bratislava, Slovakia

${ }^{40}$ Faculty of Nuclear Sciences and Physical Engineering, Czech Technical University in Prague, Prague, Czech Republic

${ }^{41}$ Faculty of Science, P.J. Šafárik University, Košice, Slovakia

${ }^{42}$ Faculty of Technology, Buskerud and Vestfold University College, Vestfold, Norway

${ }^{43}$ Frankfurt Institute for Advanced Studies, Johann Wolfgang Goethe-Universität Frankfurt, Frankfurt, Germany

${ }^{44}$ Gangneung-Wonju National University, Gangneung, South Korea

${ }^{45}$ Gauhati University, Department of Physics, Guwahati, India

${ }^{46}$ Helsinki Institute of Physics (HIP), Helsinki, Finland

${ }^{47}$ Hiroshima University, Hiroshima, Japan

${ }^{48}$ Indian Institute of Technology Bombay (IIT), Mumbai, India

${ }^{49}$ Indian Institute of Technology Indore, Indore (IITI), India

${ }^{50}$ Inha University, Incheon, South Korea
} 
${ }^{51}$ Institut de Physique Nucléaire d'Orsay (IPNO), Université Paris-Sud, CNRS-IN2P3, Orsay, France

${ }^{52}$ Institut für Informatik, Johann Wolfgang Goethe-Universität Frankfurt, Frankfurt, Germany

${ }^{53}$ Institut für Kernphysik, Johann Wolfgang Goethe-Universität Frankfurt, Frankfurt, Germany

${ }^{54}$ Institut für Kernphysik, Westfälische Wilhelms-Universität Münster, Münster, Germany

${ }^{55}$ Institut Pluridisciplinaire Hubert Curien (IPHC), Université de Strasbourg, CNRS-IN2P3, Strasbourg, France

${ }^{56}$ Institute for Nuclear Research, Academy of Sciences, Moscow, Russia

${ }^{57}$ Institute for Subatomic Physics of Utrecht University, Utrecht, Netherlands

${ }^{58}$ Institute for Theoretical and Experimental Physics, Moscow, Russia

${ }^{59}$ Institute of Experimental Physics, Slovak Academy of Sciences, Košice, Slovakia

${ }^{60}$ Institute of Physics, Academy of Sciences of the Czech Republic, Prague, Czech Republic

${ }^{61}$ Institute of Physics, Bhubaneswar, India

${ }^{62}$ Institute of Space Science (ISS), Bucharest, Romania

${ }^{63}$ Instituto de Ciencias Nucleares, Universidad Nacional Autónoma de México, Mexico City, Mexico

${ }^{64}$ Instituto de Física, Universidad Nacional Autónoma de México, Mexico City, Mexico

${ }^{65}$ iThemba LABS, National Research Foundation, Somerset West, South Africa

${ }^{66}$ Joint Institute for Nuclear Research (JINR), Dubna, Russia

${ }^{67}$ Konkuk University, Seoul, South Korea

${ }^{68}$ Korea Institute of Science and Technology Information, Daejeon, South Korea

${ }^{69}$ KTO Karatay University, Konya, Turkey

${ }^{70}$ Laboratoire de Physique Corpusculaire (LPC), Clermont Université, Université Blaise Pascal, CNRS-IN2P3, Clermont-Ferrand, France

${ }^{71}$ Laboratoire de Physique Subatomique et de Cosmologie, Université Grenoble-Alpes, CNRS-IN2P3, Grenoble, France

${ }^{72}$ Laboratori Nazionali di Frascati, INFN, Frascati, Italy

${ }^{73}$ Laboratori Nazionali di Legnaro, INFN, Legnaro, Italy

${ }^{74}$ Lawrence Berkeley National Laboratory, Berkeley, California, USA

${ }^{75}$ Lawrence Livermore National Laboratory, Livermore, California, USA

${ }^{76}$ Moscow Engineering Physics Institute, Moscow, Russia

${ }^{77}$ National Centre for Nuclear Studies, Warsaw, Poland

${ }^{78}$ National Institute for Physics and Nuclear Engineering, Bucharest, Romania

${ }^{79}$ National Institute of Science Education and Research, Bhubaneswar, India

${ }^{80}$ Niels Bohr Institute, University of Copenhagen, Copenhagen, Denmark

${ }^{81}$ Nikhef, Nationaal Instituut voor Subatomaire Fysica, Amsterdam, Netherlands

${ }^{82}$ Nuclear Physics Group, STFC Daresbury Laboratory, Daresbury, United Kingdom

${ }^{83}$ Nuclear Physics Institute, Academy of Sciences of the Czech Republic, ̌̌ež u Prahy, Czech Republic

${ }^{84}$ Oak Ridge National Laboratory, Oak Ridge, Tennessee, USA

${ }^{85}$ Petersburg Nuclear Physics Institute, Gatchina, Russia

${ }^{86}$ Physics Department, Creighton University, Omaha, Nebraska, USA

${ }^{87}$ Physics Department, Panjab University, Chandigarh, India

${ }^{88}$ Physics Department, University of Athens, Athens, Greece

${ }^{89}$ Physics Department, University of Cape Town, Cape Town, South Africa

${ }^{90}$ Physics Department, University of Jammu, Jammu, India

${ }^{91}$ Physics Department, University of Rajasthan, Jaipur, India

${ }^{92}$ Physik Department, Technische Universität München, Munich, Germany

${ }^{93}$ Physikalisches Institut, Ruprecht-Karls-Universität Heidelberg, Heidelberg, Germany

${ }^{94}$ Politecnico di Torino, Turin, Italy

${ }^{95}$ Purdue University, West Lafayette, Indiana, USA

${ }^{96}$ Pusan National University, Pusan, South Korea

${ }^{97}$ Research Division and ExtreMe Matter Institute EMMI, GSI Helmholtzzentrum für Schwerionenforschung, Darmstadt, Germany

${ }^{98}$ Rudjer Bošković Institute, Zagreb, Croatia

${ }^{99}$ Russian Federal Nuclear Center (VNIIEF), Sarov, Russia

${ }^{100}$ Russian Research Centre Kurchatov Institute, Moscow, Russia

${ }^{101}$ Saha Institute of Nuclear Physics, Kolkata, India

${ }^{102}$ School of Physics and Astronomy, University of Birmingham, Birmingham, United Kingdom

${ }^{103}$ Sección Física, Departamento de Ciencias, Pontificia Universidad Católica del Perú, Lima, Peru

${ }^{104}$ Sezione INFN, Bari, Italy

${ }^{105}$ Sezione INFN, Bologna, Italy

${ }^{106}$ Sezione INFN, Cagliari, Italy

${ }^{107}$ Sezione INFN, Catania, Italy

${ }^{108}$ Sezione INFN, Padova, Italy

${ }^{109}$ Sezione INFN, Rome, Italy 


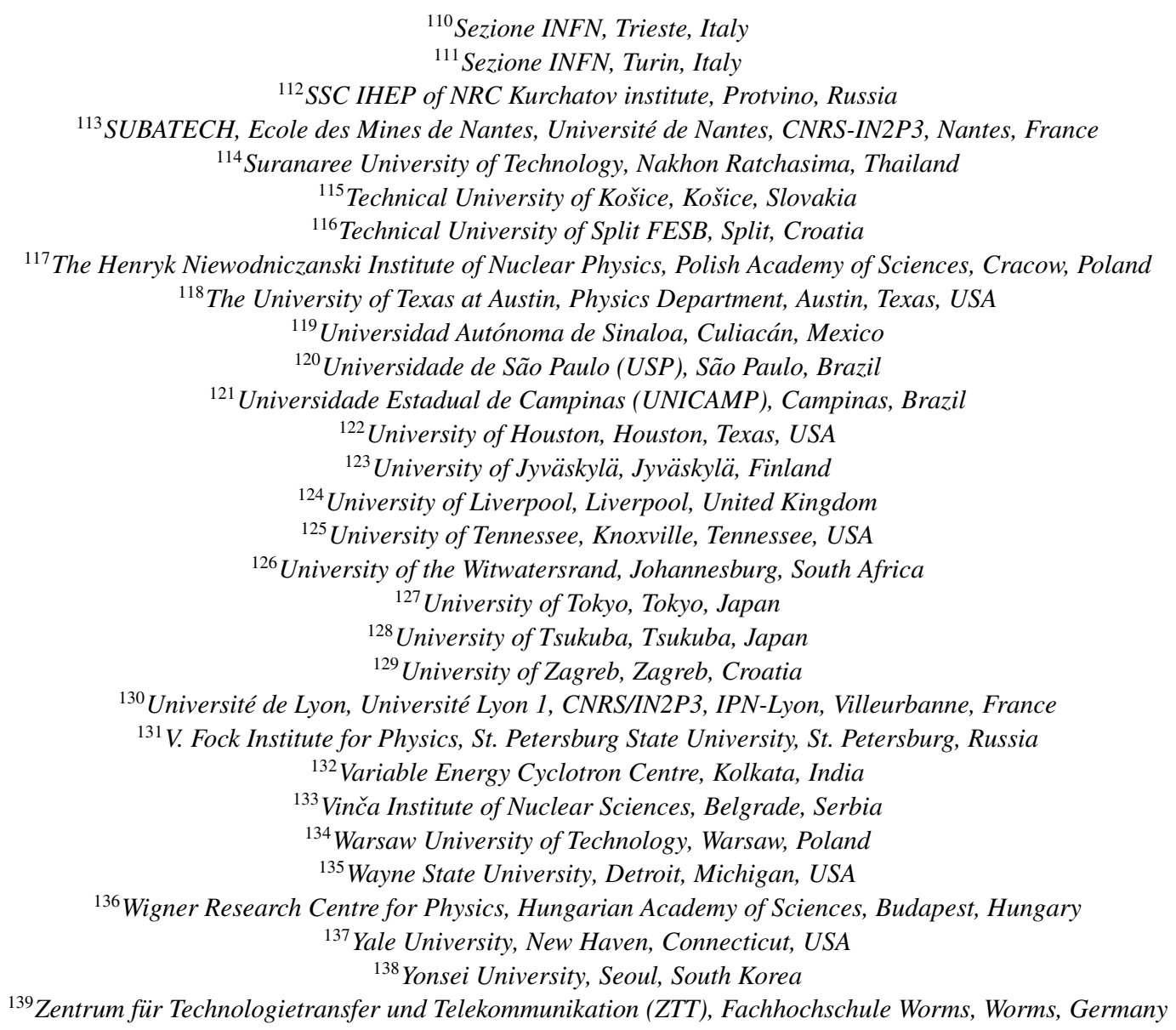

*Deceased.

${ }^{\dagger}$ Also at M.V. Lomonosov Moscow State University, D.V. Skobeltsyn Institute of Nuclear, Physics, Moscow, Russia.

${ }^{\ddagger}$ Also at University of Kansas, Lawrence, Kansas, USA. 